\title{
Mechanical transformations of living cavitary bodies
}

\author{
Valentin Borkhvardt ${ }^{1}$ \\ ${ }^{1}$ Department of Vertebrate Zoology, Faculty of Biology, Saint Petersburg State University, \\ Universitetskaya nab., 7-9, Saint Petersburg, 199034, Russian Federation; \\ borkhvardt@mail.ru
}

Address correspondence and requests for materials to Valenitn Borkhvardt

\begin{abstract}
All living organisms and many of their parts are organised in an essentially similar manner: they are closed cavitary bodies consisting of 1) the inner mass and 2) a relatively thin sheath enclosing it. This organisation allows living bodies to change shape by employing hydrostatic forces. These forces are generally recognised to govern changes of shape in walled cells. To explain transformations of other organisms, other factors are usually sought. In this paper, the hydrostatic mechanism is represented as a universal mode of shape formation. It acts in all kinds of organisms, determining the course of diverse processes such as development of cell outgrowths, limb buds, gut derivatives and sense organs; endocytosis; cell division; branching of capillaries; gastrulation; cell locomotion; muscle contraction etc.
\end{abstract}

Keywords: organisms, organs, cells, morphogenesis, evagination, invagination, cell division, cell locomotion, muscle contraction, hydromechanical model

\section{Introduction}

A great many creatures of the most diverse kinds inhabit the Earth. Some are relatively stable while others, such as amoeboid protists, are always changing shape. Major transformations of animals are associated with early stages of their ontogenesis, when their appearance is shaped and their organs form, grow and increase in complexity. What mechanisms govern the transformations of living organisms and create their diversity? I will try to answer this question using evidence from, mostly, cells, protists and animal embryos and larvae, which demonstrate the greatest variability of external and internal structure.

I will analyse the following morphogenetic processes: the development of

Citation: Borkhvardt, V. 2017. Mechanical transformations of living cavitary bodies. Bio. Comm. 62(2): 103-155. doi: 10.21638/11701/spbu03.2017.206

Author's information: Professor Valentin Borkhvardt, Ph.D., Dr. of Science, Leading Scientist, orcid.org/0000-0001-6480-9077

Translator: Natalia Lentsman

Manuscript Editor: Yegor Malashichev, Department of Vertebrate Zoology, Saint Petersburg State University, Russia

Received: January 27, 2017;

Revised: May 27, 2017;

Accepted: June 04, 2017;

Copyright: @ 2017 Borkhvardt. This is an open-access article distributed under the terms of the License Agreement with Saint Petersburg State University, which permits to the authors an unrestricted distribution and self-archiving free of charge.

Funding: The study was partially funded by Saint Petersburg University (grant no. 0.38.292.2015).

Competing interests: The author has declared that no competing interests exist. pollen tubes, fungal hyphae, cell outgrowths, limb buds, gut derivatives, sense organs; endocytosis; cell division; gastrulation in echinoderms and amphibians; cell locomotion; muscle contraction. These processes have been studied for ages, usually one process or a small group of processes at a time, and special models have been proposed in each case. I am going to propose a single model for all the cases.

Why is a single model possible? It is possible because living organisms, diverse as they may be, are basically identical: they are closed cavitary bodies, and so are many of their parts. Complex multicellular organisms are arranged in a hierarchy of cavitary bodies, and early embryos of vertebrates are perfectly suited for a case study of this hierarchy. Their primary cavity contains the coelom, the gut, the neural tube and blood vessels. Cells composing these cavitary organs or filling spaces between them are closed cavitary bodies too. In their turn, they contain cavitary organelles such as mitochondria, the Golgi apparatus and endoplasmic reticula.

Cavitary structure is a fundamental feature of living organisms. It sets them apart from inorganic natural formations, which are dense bodies (some organic structures, e.g., horny structures, are also dense).

A cavitary body consists of two parts: 1) the inner mass and 2) the sheath serving as a mechanical barrier between the inner mass and the environment (the outer 

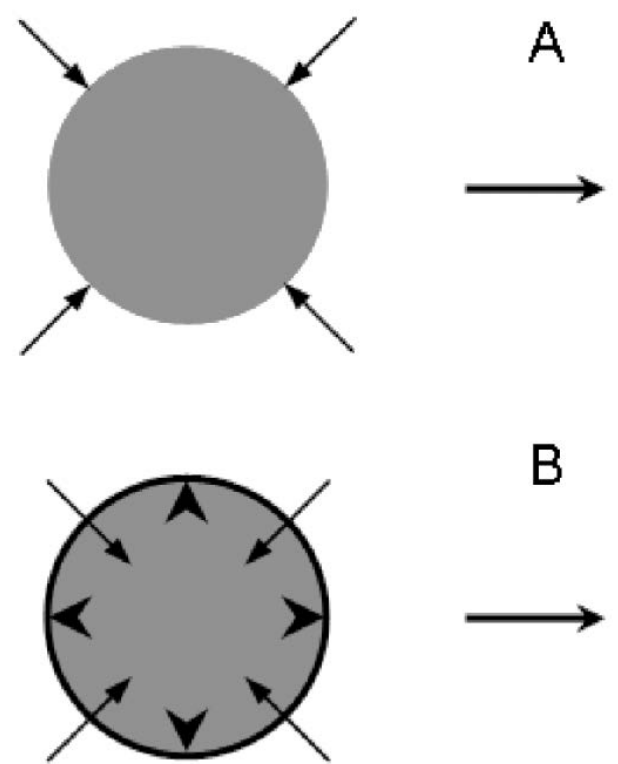

Fig. 1. Growth of a dense (A) and a cavitary (B) body (a scheme),

A: Building material is added (arrows) to the body surface (dark grey circle). At the site where a lot of the material is added (bold arrow), the body grows faster. B: Building material is deposited (arrows) under the sheath (black circumference) of the body and increases the volume of its inner mass (dark grey circle). The growing inner mass presses (arrowheads) on the sheath, displacing it off the body centre. mass). In gram-negative bacteria the sheath is represented only by the plasmalemma; gram-positive bacteria have a wall composed of peptidoglycans on the outside of the membrane. In eukaryotic cells the plasmalemma is underlain by the cortex mostly composed by actin filaments; it may be considered as an inner layer of the sheath. In plant and fungal cells there is a third layer, the outer wall, mostly composed of polysaccharides. Cells which have this layer are called walled cells, while cells lacking this layer are called unwalled cells. The sheath of animal embryos and larvae as well as the sheath of their cavitary organs consists of two layers. One of them is composed by epithelial cells, while the second layer, the basement membrane, mostly consists of collagen fibres.

Changes in the shape of bodies usually occur in parallel with changes in their size. Dense and cavitary bodies change their size in a different manner. Dense bodies (crystals, stalactites, snowballs, horny structures etc.) are enlarged by addition of building material to their surface, which becomes walled up in the process (Fig. 1A). Changes in the shape of these bodies are mostly determined by a non-uniform addition of the building material to the surface. Decrease in the size (destruction) of dense bodies such as rocks also starts at the surface. The non-uniform nature of this process, which results in the changed shape of the body, is determined, among other things, by a non-uniform impact of the environment.
Organisms and their cavitary parts grow differently (Fig. 1B). Their closed condition is not absolute since the sheath is permeable to various ions and molecules, including, importantly, water molecules. Having permeated the sheath, the new materials are added to the inner mass, increasing its volume. Some of them are also incorporated into the sheath, whose area may increase as a result. Under pressure of the growing inner mass the sheath moves away from the centre. The growth of a living body may thus be described as a centrifugal displacement of the sheath. As it moves the sheath, the inner mass has to overcome its resistance as well as the resistance of the outer mass. Some cavitary inorganic bodies may grow in a similar manner, e.g., a balloon which is being inflated. However, the area of the balloon's sheath increases only by stretching of the same material. When cavitary bodies shrink (a deflating balloon, an animal losing weight etc.) the decrease mostly affects their inner mass, and the sheath is displaced centripetally in the process.

Actions resulting in the changed shape of living cavitary bodies are, in general, referred to as evaginations and invaginations. These same terms are applied to body parts that emerge as a result. No strict definitions of the terms "evagination" and "invagination" can be found in the literature; apparently, they are considered to be self-explanatory. The following example shows that things are not as simple as they seem. During normal development of vertebrates 




Fig. 2. Hydromechanical model (a scheme).

A: A cavitary turgid body in a static state. The inner (dark grey circle) and the outer mass (light grey background) press on the sheath (black circumference) of the body uniformly but with a different force (directly proportional to the size of the arrowheads). The sheath and the outer mass restrain the expansion of the inner mass. B: The inner mass expands, presses on the sheath with a greater force (+) and displaces it centrifugally. At the site where the sheath offers the least resistance (thin part of the circumference), the inner mass expands faster - evaginates. C: The volume of the inner mass decreases, and the internal pressure drops below the level of the external pressure (-). At the site where the sheath offers the least resistance (thin part of the circumference), the outer mass invaginates into the body.

the gut derivatives grow into the primary body cavity of embryos and larvae and therefore are called invaginations. The same outgrowths of a gut cultured in vitro in a liquid medium are naturally perceived as evaginations. To avoid misunderstandings, I will now explicate the use of these terms in this paper. I will distinguish between evaginations and invaginations depending on their relation to the mother body (in the above example the mother body is the gut). I will apply the term "evagination" to any derivative that protrudes beyond the boundaries of the mother body and is filled with its inner mass (Fig. 2B). Any depression of the mother body whose sheath is part of the mother body's sheath and which is filled with the outer mass will be referred to as an invagination (Fig. 2C). In this classification, any outgrowth of the mother body is an evagination.

So, the first question concerning the mechanism of transformation of living cavitary bodies can be put as follows: what are the forces underlying the emergence and development of evaginations and invaginations? We will start the discussion with evaginations, more precisely, with evaginations of walled cells. There is an almost complete agreement in the scientific community in regard of corresponding mechanisms.

\section{Evaginations}

\section{CELLS}

Walled cells - The cell wall is a layer of extracellular matrix closely adjacent to the plasmalemma. The wall makes the sheath more resistant, and so the internal (turgor) pressure of a walled cell may be much higher than the external pressure. "In general, the process of plant cell growth is driven by the relationship between turgor pressure, controlled water uptake and mechanical cell wall resistance" (Chebli and Geitmann, 2007, p. 235). The mechanics of a cell located inside a homogenous liquid mass can be represented as follows. The liquid inner mass (the cytosol) presses against the cell's sheath uniformly at all points of its area; the outer mass also presses uniformly against it (Fig. 2A). The outer mass and the sheath, mostly the wall, resist the turgor pressure. If the summary resistance of the sheath and the outer mass counterbalances the internal pressure, the size and the shape of the cell do not change. Swelling (mostly by water uptake), the inner mass stretches the cell wall and displaces it centrifugally: the cell grows. If the wall is mechanically uniform, the cell grows without changing its shape (Fig. 1B). If the wall is mechanically non-uniform and resists differently at different points, the cell grows non-uniformly and changes the shape.

Unidirectional (apical) growth, which is, in essence, a prolonged evagination, is of the greatest interest in the light of our topic. This is how it occurs in pollen tubes of higher plants. A pollen grain, which is the precursor of the pollen tube, has a durable sheath, the sporoderm. Its inner layer, the intine, is uniform, while the outer layer, the exine, has several pores, i.e. it is mechanically non-uniform. The inner mass of the pollen grain swells due to water uptake and evaginates together with the intine through the exine at the site where the latter is perforated by a pore; this evagination signals the beginning 
of the pollen tube growth. As water uptake continues, the tube increases its inner mass and elongates. Secretory vesicles transport building material to its apex, which is used to form new parts of the plasmalemma and the cell wall at that site. The apical part is dome-shaped.

To consider the process of the cell wall transformation in more detail, let us discuss the growth of the potato pollen tube (see Parre and Geitmann, 2005). In the beginning, the cell wall at the tube apex is mostly composed by pectins. As the tube elongates, portions of the apical sheath are passively displaced backwards. Pectins are deetherified in the process, making the wall more rigid. At some distance from the apex new layers composed of cellulose and callose are added to the wall, which becomes even stiffer and thus even more resistant to the internal pressure. An artificial softening of the pectin wall with the help of pectinase resulted in a spherical apical swelling.

So, the growing inner mass pushes the wall of the pollen tube and moves forward itself only at the apex, where the newly formed soft wall offers the least resistance to the cytosol pressure. Normal tube growth is maintained owing to the balance between the turgor pressure and the stiffness of lateral and apical walls. This model agrees well with the results of experiments where pollen tubes were grown in anisotonic solutions. In hypertonic medium the turgor decreased and the growth of pollen tubes slowed down, while in hypotonic medium the turgor increased and the growth accelerated (Pierson et al., 1994; Messerli and Robinson, 2003; Zonia et al., 2006). In demonstrable experiments, pollen tubes were cultured in solution made with the use of heavy water $\left(\mathrm{D}_{2} \mathrm{O}\right)$. It is chemically identical to usual water $\left(\mathrm{H}_{2} \mathrm{O}\right)$ but the cell membrane is much less permeable to its larger molecules. Pollen tubes cultured in heavy water grew much more slowly than those cultured in usual water (Zonia et al., 2006).

Pollen tubes elongate unevenly, with periods of growth acceleration alternating with those of slowed growth. These alternations are regular and their rhythm is different in different plants. In Lilium longiflorum the growth rate fluctuated periodically from 0.1 to $0.4 \mu \mathrm{m}$ per second (Messerli et al., 1999). According to Pierson et al. (1996), growth rate fluctuations were erratic in short pollen tubes and became regular, with a period of about $23 \mathrm{sec}$, in pollen tubes longer than $700 \mu \mathrm{m}$. In some other plants long phases of slow growth lasting for 3-8 minutes alternated with short (several seconds) phases of explosive growth (Chebli and Geitmann, 2007). Smaller fluctuations may superimpose upon longer ones (Moreno et al., 2007). Growth fluctuations are thought to be associated with the changes of the turgor pressure and/or the stiffness of the apical wall under construction (see Holdaway-Clarke et al., 1997; Messerli et al., 2000; Messerli and Robinson, 2003; Chebli and
Geitmann, 2007; Moreno et al., 2007). If regular changes of the growth rate are caused by changes in the turgor, these latter changes should be equally regular. This means that growing pollen tubes can regulate the internal pressure. This is achieved, in particular, by controlled water uptake (see the above quotation from Chebli and Geitmann, 2007).

The structure and growth mode of another popular object of morphogenetic research, the root hair (Hepler et al., 2001; Cole and Fowler, 2006), corresponds in general to the description given for the pollen tube too. The growth of the hypha, the life form of fungi and oomycetes, is essentially similar to the growth of the pollen tube. The hypha is also a tube, often a branching one. Each branch grows apically, the apex being dome-shaped. Secretory vesicles bring building material to the apex; the new wall is softer than the older parts; the turgor pressure pushes the apical wall, in this way elongating the tube; the hyphae grow non-uniformly, with periods of fast growth alternating with periods of slow growth (Saunders and Trinci, 1979; Gray et al., 1990; Harold, 1990, 2002; Wessels, 1993; Lopéz-Franco et al., 1994; Money, 2001; Ma et al., 2005; Taheri-Talesh et al., 2008). The wall of the maternal hypha softens (Saunders and Trinci, 1979) or thins (Mullins and Ellis, 1974) at the sites where new branches (evaginations) are formed.

To complete the picture illustrating the uniformity of polarised growth mechanics of walled cells, I will provide some further examples. The first one considers the development of rhizoids in the Fucus alga. At the top of the rhizoid evaginating from the zygote, "...the cell wall is weakest" (Taylor et al., 1996, Fig. 10, legend), the weakness being determined by the thinning of the apical cell wall (Brawley and Robinson, 1985, Figs. 3-6; Harold, 1990). The second example is taken from the study of the yeast. In budding yeast, the cell wall softens at the site where a bud (an evagination) is formed (Cabib et al., 1989). Finally, let us consider gram-positive bacteria: "Since bacteria have no internal skeleton or muscle-like organs to create [various and often complex - VB] forms, the shapes must somewhat result from turgor pressure and the restraining forces supplied by the cell wall. Any shape other than a sphere implies asymmetries or nonuniformities in the properties of the wall" (Koch, 1990, p. 327).

To sum up, there seems to be a consensus that the non-uniform growth of walled cells, including polarised growth, is determined by the "...turgor pressure and the non-uniform biochemical composition of the cell wall" (Chebli and Geitmann, 2007, p. 235). The mechanical role of the plasmalemma of walled cells is usually not mentioned though it also offers some resistance to the internal pressure. This resistance should be lower at the sites of evaginations, if only because new material is built into the cell membrane there. The addition of material 
contributes to an increase in the area of both the wall and the plasmalemma. The model is presented graphically in Fig. 2B. I shall refer to it as the hydromechanical model (see Borkhvardt, 2002a, 2002b, 2009, 2010, 2016).

I think that this model is generally recognised largely because it is self-evident. It can be supported with numerous examples from everyday life. For instance, a football consists of an inner rubber bladder and an outer layer made of leather patches. If it is inflated, the rubber bladder is closely pressed to the cover much in the way the plasmalemma is pressed against the cell wall. The internal pressure is uniformly distributed across the entire area of the bladder and the cover, making the ball spherical. If the cover bursts, the bladder evaginates through the hole. Of course, the growth of this evagination is limited because no new material is being added either to the inner mass (air) or the rubber bladder. Some resemblance can be found between the pollen tube and a gun barrel. An increased internal pressure resulting from gunpowder combustion does not deform the steel barrel but propels the bullet (an analogue of the weak apex of the pollen tube). I could think of numerous other examples of everyday processes resembling non-uniform growth, in particular, the apical growth of walled cells.

Though the apical growth model is both well-grounded and intuitively obvious, some doubts about its infallibility have been expressed. Even its erstwhile supporters have had misgivings (compare Harold, 1990 and Harold, 2002). The doubts were caused by the report that the hyphae of oomycetes (Saprolegnia, Achlya) could grow at very low or even zero turgor (Money and Harold, 1993; Harold et al., 1996). What can move the cell apex if not the pressure of the liquid inner mass? According to a popular model developed for animal cells, the sheath is pushed by bundles of actin filaments (see below). In oomycetes actin filaments, indeed, reach the very apex (Jackson and Heath, 1990, text and Fig. 6) though the authors believed that the apical actin cap served only for the reinforcement of the weak apical wall. In pollen tubes the bundles of actin filaments do not reach the apex at all (Miller et al., 1996; Taylor and Hepler, 1997; Vidali and Hepler, 2001; Cai and Cresti, 2009). The only remaining variant is that the side walls of the tube elongate by themselves, without the influence of the inner mass (naturally, the inner mass has to increase proportionally, otherwise there will be partial vacuum inside). However, Hepler et al. (2001, p. 161) noted that "Growth is restricted to the tip of the dome, with no elongation occurring along the sidewalls." Generally speaking, it is hard to imagine that an independent growth of the walls can create a perfectly hemispherical dome-shaped apex of a pollen tube, a fungal hypha or an arising yeast bud. On the other hand, it is easy to imagine that such a shape results from the uniform pressure of a liquid inner mass.
Unwalled cells - I will start the discussion of animal cells and protists with another quotation indicative of the current state of views on the morphogenetic abilities of walled cells: "In physical terms, cell shape and size are governed by the mechanics of the cell wall..." (Cosgrove, 1997, p. 1031). The belief that the stiff wall plays the key role in the mechanical transformations of plant and other similar cells is strengthened by the fact that after the removal of the wall "naked" cells (protoplasts) become spherical, i.e. in the absence of the wall they no longer look like normal cells. When protoplasts do create complex shapes, it is thought to happen under the impact of some special factors. For instance, Steinberg (2007) described naked mutant Neurospora (fungi) producing pseudopodia-like outgrowths. He was quite sure that these outgrowths could not be produced by forces usually acting in walled cells: "The formation of cellular extensions in the absence of a cell wall indicates that internal cytoplasmic forces are generated that might involve the cytoskeleton" (Steinberg, 2007, Fig. 5, legend). This viewpoint is thought to be even justified as regards initially naked animal cells and unicellular organisms. Citing the authors who admitted the possibility of hydrostatic forces participating in the formation of outgrowths of animal cells, Harold (1990, p.413) wrote: "If it is true, as Oster and Perelson (208) have recently argued, that motive power is supplied by a gradient of hydrostatic pressure, the gulf that apparently separates morphogenesis in walled and unwalled cells will narrow still further." The gulf between walled and unwalled cells! The belief that such a gulf exists is firmly rooted in many a scientific mind. But is that true?

Unwalled cells are turgor bodies, as are walled cells. It is true that the turgor of unwalled cells is weak but this is a quantitative difference rather than a qualitative one. There are similarities between unwalled and walled cells in other respects, too. Animal cells, as well as plant and fungal cells, exchange water with the environment and regulate this exchange. In this way, they regulate their volume. Regulatory abilities of cells were shown in experiments: cells deformed in hypo- or hypertonic solutions actively restored their volume and shape (Mast and Hopkins, 1941; Grinstein et al., 1984; Eveloff and Warnock, 1987). Under normal conditions the cell volume is not constant either, changing, for instance, in response to various stimuli (Hoffmann et al., 2009). O’Neill (1999) emphasised that mammalian cells changed their volume in isotonic medium and considered this ability as a basic feature of these cells. According to Cala (1980, p.684), "...volume regulation is a fundamental cellular process .... It is described with the use of particular terms: regulatory volume decrease (RVD) and regulatory volume increase (RVI).

Specialised membrane channels called aquaporins have an especially large impact on the water exchange, 
accelerating manifold water transport across the plasmalemma (Wehner et al., 2003; Verkman, 2005; Verkman et al., 2006; Papadopoulos et al., 2008). Aquaporins have been found in all living organisms including prokaryotes (Engel et al., 2000; King et al., 2000; Maurel and Chrispeels, 2001). They are present even in the sheath of organelles, e.g., in the tonoplast of vacuoles in plant cells (Chrispeels and Maurel, 1994; Chaumont et al., 1998).

The discovery of aquaporins has heightened the interest in the role of water in mechanical transformations of unwalled cells. The lion's share of attention is given, however, to the cytoskeleton, especially to the active operations of actin filaments, which they perform on their own or in cooperation with myosin filaments. Pollard (2000) succinctly expressed the prevailing sentiments by putting the words "Actin everywhere" into the title of a section of his review of contractile systems. But is the belief in the active mechanical role of the cytoskeleton in the life of unwalled cells actually justified?

The factor most often included into various models is actomyosin contraction. "Practically all of the investigators preceding Berthold ('86) held that movement in all animals is due to contraction similar to that found in muscle fibers" (Mast, 1926, p. 348). Since in the past protists were also considered as animals, the "muscular" basis of movement was ascribed to an extremely broad range of organisms. Muscle contraction as such has long been found to be associated with actin and myosin filaments, while a sliding filament model has explained how they acted in striated muscle fibre. When actin and myosin filaments were found in non-muscle cells, an old idea found a new expression: "The main force-generating mechanism in both muscle and non-muscle cells seems to be actomyosin contraction..." (Strohmeier and Bereiter-Hahn, 1987, p. 631). Is there any other evidence supporting the actomyosin nature of contractile actions of non-muscle cells except the example of muscle contraction?

To begin with, let us consider the experiments that showed that the cytoplasm of fibroblasts stripped of the plasma membrane could contract after addition of calcium ions to the solution; spherical demembranated cells contracted symmetrically, while previously stretched ones shortened along the axis of prior stretching (Izzard and Izzard, 1975). The authors drew an analogy between these transformations and the contraction of muscle fibres, presuming that they were also based on actin and myosin filaments. However, they did not determine the mode of arrangement and interaction of these filaments. Now the cytoskeleton structure in non-muscle cells is much better known, and we have to admit that it is quite different from that in muscle cells.

Contractile units of striated fibres, the sarcomeres, are highly ordered systems. Actin and myosin filaments in them are arranged in parallel, and it was observed di- rectly that when the muscle contracts, they slide against each other. For this reason, the entire modern theory of muscle contraction is referred to as the sliding filament model. Most authors agree that actomyosin complexes of non-muscle cells do not have a sarcomere organisation. In the cortex, for instance, they are usually arranged in a network and no sliding of the filaments is observed. Verkhovsky et al. (1995, Fig. 8d) found sarcomere-like units of actin and myosin filaments in the outgrowths of fibroblasts and supposed that they might be involved in the retraction of lamellipodia; however, no special observations of the complexes' behaviour were made. In studies dealing with sarcomeres their size parameters are registered very precisely, with the distances between myofilaments, the length of myosin heads, the magnitude of working stroke etc. being measured with an accuracy of almost a nanometre. On the contrary, no size characteristics are given in descriptions of actomyosin in non-muscle cells. Finally, in contrast to the filaments of the sarcomere, actin filaments of non-muscle cells are unstable: they are being constantly disassembled and polymerised again.

The so-called stress fibres, which are special bundles of actin and myosin filaments, are thought to have certain similarities with sarcomeres (Sanger et al., 1983). Filaments of stress fibres are not arranged strictly in parallel to each other (e.g., Katoh et al., 1998, Fig. 11a) but the bundles extracted from the cells may shorten and narrow in the presence of ATP (Katoh et al., 1998, 2001). According to an extreme viewpoint, "Stress fibers play a central role in adhesion, motility, and morphogenesis of eukaryotic cells..." (Hotulainen and Lappalainen, 2006, p. 383). The "central role" is a patent exaggeration, however. Actually, this role is simply unclear. Hotulainen and Lappalainen (2006) admitted that stress fibres were better developed in immobile cells than in mobile ones. Isenberg and colleagues (1976) did not find any direct connection between the shortening of stress fibres and that of the entire cell, concluding that these bundles were not the only structures responsible for cell contraction. Dictyostelium and neutrophils have no stress fibres but this does not prevent them from moving and actively changing shape (Friedl et al., 2001). Fibroblasts with artificially destroyed stress fibres exhibited an even greater locomotor and morphogenetic activity than fibroblasts in the control (Höner et al., 1988). Burridge (1981) did not deny the contractile abilities of stress fibres but thought that they were not implemented during locomotion.

The conviction that actomyosin of non-muscle cells has contractile properties seems like faith. For instance, Charras et al. (2006) admitted that the cortex did not have a sarcomere organisation and that it was unclear how actin and myosin filaments interacted in it. Nevertheless these authors did not doubt the contractile abil- 
ities of the cortex. Brown and Bridgman (2003, p.425) did not question the contractile ability of actomyosin either, though they noted, describing the development of the growth cone of the neurite, that: "...a model that explains the site of force generation or molecular details of the actin-myosin interaction has been lacking." We will come across many similar statements later.

In summary, nobody knows how actomyosin of non-muscle cells contracts but all (or many) are firmly convinced that it does contract. This belief, dating back to the remote past, is quite convenient since the "actomyosin" factor comes in useful whenever one has to explain various transformations of cells. Muscle contraction, as understood by the sliding filament model, testified in its favour. At the same time, the idea that the actin filaments, by themselves or in actomyosin complexes, perform mechanical operations is also supported by some sound evidence from experiments in which the cells were treated with inhibitors of filamentous actin, first of all, cytochalasins. Cytochalasin prevents actin monomeres from binding to the barbed ends of filaments, i.e. prevents them from growing. Since filaments are depolymerised at the opposite ends, the amount of filamentous actin in the cell decreases. At the same time, the cells change shape, their locomotor or growth activity is decreased, cytokinesis is blocked and so on.

The results of experiments employing cytochalasins are contradictory. Different authors often arrived at the opposite conclusions, especially as concerns the results of treatment with cytochalasin B (see Holtzer and Sanger, 1972; Burgess and Grey, 1974; Miranda et al., 1974; Isenberg et al., 1976; Bliokh et al., 1980; Marsh and Letourneau, 1984). It was found to have a non-specific action already in the first years of its use (see Burnside and Manasek, 1972). It gradually transpired that, besides its other effects, cytochalasin B directly affects the plasmalemma (see Sanger and Holtzer, 1972; Burgess and Grey, 1974; Everhart and Rubin, 1974; Miranda et al., 1974), in particular, glucose transporters (Holtzer and Sanger, 1972; Loike et al., 1993) and $\mathrm{Na}^{+}, \mathrm{K}^{+}$, and $\mathrm{Cl}^{-}$channels (Janmey, 1998; Mazzochi et al., 2006). Cytochalasin D may also affect $\mathrm{Cl}^{-}$channels (Suzuki et al., 1993; Schwiebert et al., 1994) and glucose transport (Tsakiridis et al., 1994). Glucose and monovalent ions govern the osmotic transport of water across the cell membrane, and the influence of cytochalasins on water transport across the plasmalemma has indeed been recorded (Loike et al., 1993; Mills et al., 2000). This fact jeopardises the reliability of the cytochalasin method for the study of the mechanical role of actin filaments since the influx and efflux of water affect the cell volume and, as exemplified by walled cells, may influence their shape. Indeed, cells treated with cytochalasin B decreased in volume (Hsu and Becker, 1975; Koshy et al., 1996) or lost the ability to regulate it (Cornet et al., 1993; Pedersen et al., 2001). Cytochalasin D arrested the process of RVD and RVI (Lang et al., 1998; Petrunkina et al., 2004; Hoffmann et al., 2009). The presence of cytochalasin D in the water surrounding sea urchin embryos considerably accelerated the decrease of their volume under the influence of compression (Davidson et al., 1999); the volume decreased, of course, as a result of water loss.

Summing up the first two decades of the "cytochalasin era", Cooper (1987, p. 1475) wrote: "To understand the role of actin in cell motility, one would like probes that are specific for actin and affect only one aspect of actin's polymerization or interaction with other proteins. Although cytochalasins are the best available probes, they do not satisfy these criteria fully." According to this author, cytochalasin B, for instance, should not be used to study cell motility. Later this idea was expressed in an even more general manner (Mills et al., 2000, p. 209): "...caution must be exercised in the use of cytochalasins to probe the role of F-actin in cellular function." These authors suspected that different cytochalasins (B, D and E) might change the shape of cells even without affecting actin filaments (Mills et al., 2000).

In the 1980s latrunculins (A and B) became a usual tool in studies of the mechanical role of actin filaments (Schatten et al., 1986; Spector et al., 1989). Similarly to cytochalasin, latrunculin prevents actin polymerization but it does so differently, by binding globular actin. Latrunculin seems to have a more specific effect (Morton et al., 2000; Baluška et al., 2001) but it also affects the transport of sodium and chlorine across the plasmalemma as well as intracellular transport of aquaporins (channels facilitating water transport across the cell membrane) (Mazzochi et al., 2006). Latrunculin alters the permeability of corneal endothelium (Peterson et al., 2000) and, similarly to cytochalasin, affects the regulation of the cell volume (Hoffmann et al., 2009).

In many instances cytochalasins do not seem to be the best choice for the study of mechanical functions of actin, and latrunculins are unlikely to yield much better results. Let us suppose, however, that an absolutely specific actin inhibitor has been found. Alas, it is far from certain that such an ideal tool would reveal actin's mechanical function. There are indications that actin filaments affect the distribution of membrane proteins (Paller, 1994) and the functioning of ion channels (Cantiello et al., 1993; Schwiebert et al., 1994; Wang et al., 1998; Koch and Korbma-cher, 2000; Schwab et al., 2007; Hoffmann et al., 2009). Ahmed et al. (2000) thought that cytochalasin $\mathrm{D}$ and latrunculin activated $\mathrm{Cl}^{-}$channels precisely through the destruction of the actin cytoskeleton, which normally inhibits them (see also Lang et al., 1998; Mazzochi et al., 2006). According to Mills and Mandel (1994, p.1164), "The cytoskeleton, so named because of its role in cell shape and morphological organization, may also play a key role as a regulator of 
membrane transport events." Activation or inhibition of the ion channels may affect the water exchange of cells and, further, their volume and shape. Baluška et al. (2001) considered the involvement of actin cytoskeleton in the elongation of plant cells in this light. The cortical F-actin might control the fusion of secretory vesicles with the cell membrane (Bachewich and Heath, 1998), i.e. affect the transport of building material to its growing parts. Papadopoulos et al. (2008) noted that polymerization or depolymerization of actin decreased or increased, respectively, the osmotic concentration of the cytosol, affecting in this way the cell's water exchange.

To sum up, the results of experiments employing cytochalasins and latrunculins allow different interpretations. Viewed in a certain light, they can strengthen the case for the hydromechanical model. Importantly, no one doubts that the inhibitors do destroy the actin cytoskeleton. At the same time, we should remember that actin filaments compose much of the cortex, and their state may affect the mechanical properties of the cell sheath. These properties, in turn, affect morphogenetic processes, as shown by evidence from walled cells. In this respect, the results of experiments with cell treatment with cytochalasin and latrunculin are certainly relevant for our discussion.

Though I am sceptical about active mechanical actions of the cytoskeleton of non-muscle cells, I am not going to discard this possibility yet. The hypotheses employing the factor of active cytoskeleton for the explanation of morphogenetic processes will be considered alongside with the others.

After these preliminary remarks, let us address, at last, the evaginations of unwalled cells. They may produce diverse outgrowths as indicated by the variety of names used for their description. I will mostly discuss structures of four types: 1) cylindrical microvilli covering the surface of stationary cells; 2) flat and often very broad lamellipodia, especially characteristic of growing cells (e.g., growing neurons) and crawling cells; 3) cylindrical or slightly conical filopodia produced by lamellipodia; 4) blebs, which may arise on the surface of various cells in various states. We will begin our discussion concerning the development of cell outgrowths with these blebby evaginations.

"The formation of blebs can be assumed to result from the internal pressure of the cells and local weakenings in the cortical complex, caused either by loosening of the connection between the plasma membrane and the cortical fibrillar net, or by weakening of the net structure itself" (Bereiter-Hahn et al., 1990, p. 185). I would like to add that the "internal pressure" (the pressure of the liquid cytosol upon the plasmalemma) is, of course, uniform. Similar schemes, varying only in details and the degree of clarity, have been suggested by many authors (Godman et al., 1975; Tickle and
Trinkaus, 1977; Shimizu, 1990; Fedier and Keller, 1997; Keller and Eggli, 1998; Yoshida and Inouye, 2001; Charras et al., 2005, 2006; Langridge and Kay, 2006; Paluch et al., 2006; Yoshida and Soldati, 2006; Fackler and Grosse, 2008). This scheme corresponds quite well to the model developed for walled cells (the one I call the hydromechanical model): a uniform pressure of the inner mass on the sheath and a non-uniform resistance of the sheath. The cell wall being absent, the regulation of the sheath's mechanical state is taken up by the cortex, mostly consisting of actin filaments. At the sites where cortex is locally destroyed or detached from the membrane, the sheath weakens and evaginations form. We see that the cytoskeleton plays an important mechanical role in this process. It is, however, a passive role.

I have not come across any objections to the idea that the blebs grow under the uniform pressure of the liquid cytosol mass. The shape of blebs appears to be a weighty argument. Starting to evaginate as hemispheres, they may later reach an almost perfectly spherical shape (see, e.g., Cunningham, 1995, Fig. 4; Shi et al., 2005, Fig. 1).

According to the hydromechanical model, an increase of the intracellular pressure is a necessary condition for the development of evaginations (Fig. 2B). In walled cells the pressure increases mostly due to water uptake. An increased pressure inside unwalled cells is often considered to be associated with the contraction of the cortex, which squeezes the cell. This was suggested, for instance, by Yoshida and Inouye (2001) who observed blebbing in Dictyostelium. However, there could be no contraction of the cortex in the experiment of these authors because the cells were treated with cytochalasin and thus had to lose actin filaments. Fukui et al. (1990) observed formation of various evaginations including blebs in mutant Dictyostelium cells devoid of myosin filaments, i.e. devoid of actomyosin complexes. Based on this, the authors concluded that forces generated by myosin were unnecessary for the formation of the outgrowths. On the other hand, Fedier and Keller (1997, p. 335), who grew carcinosarcoma cells in hypertonic medium, concluded that "...the reduction of free water within the cells... is the direct cause for inhibition of blebbing and locomotion." In the experiment of Brette et al. (2000) the fibres of the rat heart muscle placed in hypotonic medium increased in volume (swelled) and large blebs appeared at their surface. Thus, the influx of water into the cell and an increase in the cytoplasmic volume are the most plausible reasons of an increase of intracellular pressure and the subsequent development of blebby evaginations.

It has been suggested that the hydrostatic pressure determines the formation not only of blebs but also of other cell outgrowths. Observing the behaviour of spermatozoa of echinoderms in the water of varying 
tonicity, Tilney and Inoue (1985) found that the rate of elongation of their acrosomal processes was higher in hypotonic medium and lower in hypertonic medium; when the osmolality of the solution was high, the processes stopped to grow altogether. Based on these data, Oster and Perelson (1987) decided that the force elongating the acrosomal process was the hydrostatic pressure, which increased due to the water influx into the cell. The authors took into account that this pressure had to spread uniformly in all directions. In their opinion, longitudinal actin filaments assisted in maintaining the cylindrical shape of the outgrowths. In this way, they ascribed the cytoskeleton an important but a passive role in the morphogenesis.

Oster and Perelson (1987) promulgated their hypothesis expanding it to various outgrowths. They were not alone in that. Fedier and Keller (1997, p. 326) did not discriminate between various evaginations, either: "...hydrostatic pressure rather than actin polymerization at the front is the direct force driving the membrane forward during bleb formation. Cells with lamellipodia show a similar response to hypertonic media, suggesting that basically similar mechanisms may operate in both forms of protrusions." The same view on the growth of lamellipodia was expressed by Bereiter-Hahn et al. (1981) and Strohmeier and Bereiter-Hahn (1987).

Numerous experiments have been performed showing that hypertonic medium inhibits the formation of evaginations while hypotonic medium stimulates it. There are indications that hypotonic medium promotes the formation of outgrowths in fibroblasts (Carton et al., 2003). Forscher et al. (1987, Fig. 10, legend) observed that "Filopodia [of the growth cone - VB] rapidly extend and swell immediately after $\mathrm{K}^{+}$treatment..." $\mathrm{Ob}$ serving neurite growth in anisotonic solutions, Bray et al. (1991, p.507), on the contrary, decided that their data "... argue against an osmotic mechanism for growth cone extension and show that the growth of filopodia, in particular, is unlikely to be driven by osmotically generated hydrostatic pressure." In general, the results of experiments by Bray et al. (1991) are ambiguous. Only filopodia grew in hypertonic medium and for a short time only. After that they shortened, while lamellipodia contracted in the very beginning. On the other hand, in hypotonic medium entire neurites grew at a higher rate; moreover, they branched and each branch developed a new growth cone bearing lamellipodia and filopodia. The authors admitted that "It is possible that under normal conditions the elongation of the axonal cylinder may be derived in part from a small positive internal pressure" (Bray et al., 1991, p.515).

The appearance of evaginations other than blebs also indicates that they may be formed by the cytosol pressure. Microvilli, similarly to pollen tubes or fungal hyphae, look like regular cylinders with a dome-shaped apex (see electron micrographs: Mooseker and Tilney, 1975; Chambers and Grey, 1979; Hirokawa et al., 1982). Filopodia may also look like this (see Bridgman and Dailey, 1989, Fig. 2; Lewis and Bridgman, 1992). Acrosomal processes of echinoderm spermatozoa are cylindrical with a swollen tip and local swellings sometimes arise along them (Tilney and Inoué, 1982, 1985). Similar swellings may arise at neuritic shafts (Aletta and Greene, 1988) and on microvilli, which then have a beaded appearance (Chandler and Heuser, 1981). The apex of the microvilli may swell, too, so that they become clavate (Godman et al., 1975). Swellings were observed at the apex of the filopodia of the neuritic growth cone (Yamada et al., 1971). Even broad and flat lamellipodia have some structural elements suggesting the influence of the uniform pressure of the liquid cytosol on their formation: at the vertical section their leading edge is rounded, resembling the apex of microvilli (Radice, 1980, Fig. 6; Bereiter-Hahn et al., 1981, Figs. 7-9).

The location of blebs is determined by a local weakening of the sheath due to the destruction of the cortex and/or the loosening of its association with the cell membrane (see above). Newly formed blebs have little submembrane actin (Keller and Eggli, 1998; Charras et al., 2006; Fackler and Grosse, 2008); it can be seen at electron micrographs that the sheath of blebs is much thinner than in other areas of the cell (Fay and Delise, 1973, Fig. 6; Kargacin and Fay, 1987, Fig. 9). Similar observations were made on evaginations of other types. Bereiter-Hahn and Lüers (1998) found a weakening of the connections between cortical filaments and the peripheral plasmalemma in lamellipodia of keratocytes. The destruction of the cortex or its detachment from the membrane was observed at the leading edge of moving cells (Stossel, 1993), during formation of evaginations in amoebae (Stockem and Kłopocka, 1988; Grębecki, 1990), Dictyostelium (Merkel et al., 2000; Yoshida and Inouye, 2001), the protist Vampyrella lateritia (Hülsmann and Grębecki, 1995), macrophages, astrocytes and thyroid cells (see Grębecki, 1994). It seems that only Keller and Eggli (1998) contradistinguished lamellipodia and blebs indicating that in carcinosarcoma cells the association between the plasmalemma and the cortex did not loosen at the edge of lamellipodia.

Forming outgrowths of Dictyostelium described by Yoshida and Inouye (2001) demonstrate a clear constructive similarity with pollen tubes or fungal hyphae. This is how these outgrowths behave at stages 4 and 5 (Fig. 13, legend): "(4) Contraction of the cortical layer continues to force the cytosol into the protrusion. The protrusion expands because of the low tension of its membrane, which lacks a cortical layer of F-actin. (5) A cortical layer of F-actin ... extends from the cell body into the protrusion along its lateral membrane, but the absence of F-actin cortex at its distal region allows its further elongation." At 
stage 4 the outgrowth lacking the cortex is blebby and at stage 5 (and during subsequent elongation), after its lateral walls have been strengthened with actin cortex, it is cylindrical. As we see, the cytoskeleton plays an important but a passive role in this case as well. To remind, in the experiment with the use of cytochalasin the outgrowths of Dictyostelium cells, which lacked the actin cortex, looked like blebs (Yoshida and Inouye, 2001).

Thus, the scheme developed for walled cells (Fig. 2B) may be applied, in good faith, to unwalled cells as well. However, most authors seem reluctant to extend this model to cylindrical and flat outgrowths though they fail to explain why. May be they simply find it difficult to imagine that the uniform pressure of the liquid cytosol can create non-spherical shapes and ignore the arguments presented by the followers of the "hydrostatic" solution, e.g., the idea about a passive strengthening role of central or peripheral actin filaments. Whatever the case, the dominant opinion is that during the development of microvilli, filopodia and lamellipodia bundles of actin filaments do most of the job. Elongating by addition of monomeres at the distal ends, they push the plasmalemma and thrust it forward (see Abraham et al., 1999; Borisy and Svitkina, 2000; Sheetz, 2001; Fenteany and Zhu, 2003; Grimm et al., 2003; Pollard, 2003; Pollard and Borisy, 2003; Ridley et al., 2003; Ponti et al., 2004; Small and Resch, 2005; Yamazaki et al., 2005; Mogilner, 2006; Conti and Adelstein, 2008; Iwadate and Yumura, 2008; Le Clainche and Carlier, 2008). (This model cannot be applied to blebs since actin bundles are absent there - see Keller and Eggli, 1998; Torgerson and McNiven, 1998; Shi et al., 2005).

It follows from all this that while the world of walled cells is governed by a single morphogenetic mechanism, in the world of unwalled cells evaginations are created in at least two different ways. To note, the same cells may form different and, in addition, highly variable outgrowths. For instance, small spines at the end of developing dendrites have diverse and often bizarre (e.g., mushroom-like) shapes (Parnass et al., 2000; Hering and Sheng, 2001). Blebs often arise near microvilli (Sheetz, 2001) and at the leading edge of moving cells where they may reversibly transform into lamellipodia (Keller and Eggli, 1998; see also Flanagan et al., 2001). This does not seem to perturb the researchers, however. For instance, Borisy and Svitkina (2000, p. 109) admitted the possibility that "It is not at all unreasonable to suppose that multiple mechanisms for cellular protrusion exist, with certain ones being dominant in particular cellular contexts." A similar opinion has been suggested by Heidemann et al. (1990), Munevar et al. (2001), Yoshida and Inouye (2001), Langridge and Kay (2006). Yet I fail to see any particular reasons in favour of the idea of multiple mechanisms and, in particular, the "pushing filament" hypothesis.
The idea that actin filaments have a direct impact on the apex of cell outgrowths is based on a more general view that the cytoskeleton plays an active mechanical role in the life of cells. However, this view or, rather, its supporting evidence should be treated at the very least with caution. The "pushing filament" hypothesis, it would seem, was supported (see Borisy and Svitkina, 2000; Mogilner, 2006) by the studies of movement of Listeria bacteria inside cells. Actin filaments near the bacterium form a long "tail" extending in the direction opposite to that of its movement. Tilney and Portnoy (1989) suggested that the tail filaments pushed the bacterium and tested their idea by treating the cells containing the bacteria with cytochalasin $\mathrm{D}$. This method, however, does not seem to be reliable in such a case (see above). Moreover, the authors ignored their own observations that at some stretches the bacterium moved in the cells without any actin "tail" at all (see Tilney and Portnoy, 1989, Fig. 23).

While strong evidence in favour of the model of pushing filaments is basically absent, many of its aspects are questionable. There are numerous indications that the arrangement of actin filaments is not strict in cell outgrowths. For instance, while the central filaments in mature microvilli are arranged in longitudinal bundles, at the early stage of the outgrowths' development they usually form an unstructured network (Chambers and Grey, 1979; Tilney and Jaffe, 1981). Bundles of filaments were absent both in thin processes (Savion and Selinger, 1978) and in lamellar evaginations (Andrews, 1976). Schroeder (1972) observed almost no filaments in the microvilli covering the sea urchin zygote. "In untensed, forward-moving lamellar protrusions [of epithelial cells - VB], microfilaments appear disorganized and anisotropically oriented" (Kolega, 1986, p. 1400). Begg et al. (1982, p.24) thought that "...formation of actin filament bundles is not necessary for MV [microvilli at the surface of the sea urchin zygote $-\mathrm{VB}$ ] elongation but is required to provide rigid support for MV." Indeed, the microvilli that had no longitudinal filament bundles ceased to be regular cylinders (Savion and Selinger, 1978; Chambers and Grey, 1979). In the experiments of Marsh and Letourneau (1984), neurites treated with cytochalasin B did not form lamellipodia and filopodia but grew and branched; no organised actin filaments were observed in their apical parts. In amoebae (Sarcodina) actin filaments concentrate in the cortex and are lacking in the endoplasm. However, crawling amoebae easily form pseudopodia while floating amoebae produce long fine outgrowths. Forams, which are closely related to amoebae, can form especially long pseudopodia-like outgrowths called rhizopodia.

In the cases when actin filaments are indeed arranged in central bundles extended towards the apex of the outgrowths, we cannot be sure that they actually 
butt against the apical membrane. Spatial relationships between filaments and the plasmalemma are perhaps best studied in microvilli. One can judge about the results by the following quotation: "Although electron microscopy is perhaps the best assay for identifying specific interactions of filaments with membranes, there is no evidence, as yet, which demonstrates a physical connection between core filaments and the membrane [the apical membrane of microvilli - VB]. We can only say that the barbed ends of core filaments are embedded in a densely staining region of cytoplasm which in turn is very close to the plasma membrane" (Mooseker et al., 1982, p. 233). According to Lewis and Bridgman (1992), actin filaments in the lamellipodia of a neurite growth cone contact the apical plasmalemma by means of some amorphous or globular material. The question, however, is whether even the electron microscope can reveal an absolute contact. Should there be a gap the length of a monomer (or even less), the filament would not be able to push the membrane.

The problem of contact has another aspect. If there is contact, how do actin monomeres incorporate themselves between the filaments and the cell membrane? It has been suggested that the plasmalemma is in constant Brownian motion, and when it moves centrifugally the actin monomeres bind to the distal ends of filaments (see Mogilner and Oster, 1996). Effectively, this means that the cell membrane moves "by itself" while the filaments only support it at the new place. In a later model (Mogilner and Oster, 1996) Brownian motion was ascribed to the ends of the filaments, which in lamellipodia (Small et al., 1995, 2002; Borisy and Svitkina, 2000) approach the membrane at an oblique rather than at a right angle. Loitto et al. (2002, 2007), having found numerous aquaporin channels in the membrane of filopodia, suggested another mechanism possibly resulting in oscillations of the plasmalemma: an intense influx of water via aquaporins raises hydrostatic pressure in the filopodia of moving cells, which breaks the contact of filaments with the membrane. The authors suggested this mechanism in order to support the pushing filament model; essentially, however, they transferred the role of the driving force to the cytosol pressure.

Spermatozoa of nematodes (Ascaris, Caenorhabditis), which have almost no actin, crawl by producing a large pseudopodium with numerous small evaginations at its surface (Nelson et al., 1982; Roberts and Streitmatter, 1984). These evaginations were described as fingerlike projections ("villipodia") by Sepsenwol et al. (1989), while at some micrographs in another study they looked like blebs (Nelson at al., 1982, Fig. 1). The pseudopodium is filled with very fine filaments (2-3 nm thick) composed by a special protein (major sperm protein, MSP). The filaments are arranged into fibre complexes, which enter the villipodia and are somehow involved in the movement of spermatozoa (Sepsenwol et al., 1989). Fibre complexes may elongate in vitro, and it has been suggested that they push and evaginate the plasmalemma of spermatozoa, i.e. functionally substitute actin bundles (Roberts and Stewart, 2000; Bottino et al., 2002). This hypothesis expanded the applicability of the "pushing filament" model but did not provide any new arguments in its favour.

Finally, it should be remembered that not only cells but also their cavitary organelles can change shape. Mitochondria are highly variable organelles (in particular, they may look like branching tubules - Hermann and Shaw, 1998; Yaffe, 1999), and so are vacuoles of plant cells (Chaumont et al., 1998). Ovečka et al. (2005) described and photographed (Fig. 2d) a vacuole of the root hair whose long finger-like projections jutted into the subapical zone; the projections were unstable, changing shape constantly (see also Cole and Fowler, 2006, Fig. 1b). There are no actin filaments inside mitochondria and vacuoles (while aquaporins are present in the tonoplast!).

Comparing the "pushing cytosol" model and the "pushing filament" model, we can see that the argumentation in favour of the former is more rigorous. The pushing cytosol model, apart from being self-evident, may rest upon a generally accepted view concerning the morphogenesis of walled cells. Evidence from blebs shows that the hydrostatic mechanism operates in unwalled cells as well. The pushing filament model, on the other hand, cannot be supported by any analogy. The experiments with the use of cytochalasins and latrunculins were thought to be a solid enough basis of this model but as we have seen, these results may be interpreted in favour of the hydrostatic mechanism, too. At the same time, the hydromechanical model (Fig. 2B) does not encounter the difficulties faced by the pushing filament model. Bundles of actin filaments may be absent yet the cytosol is always there. It is unclear whether the filaments touch the plasmalemma yet the liquid cytosol always touches it.

Doubts about the broad occurrence of the hydrostatic mechanism may be rooted in the belief that the uniform pressure of the liquid cytosol should create spherical shapes. Indeed, why do many evaginations look like cylinders or lamellae rather than blebs? To remind, several authors suggested that central bundles of filaments might assist in maintaining the tubular shape of the outgrowths (Begg et al., 1982; Oster and Perelson, 1987). This idea was not supported by any special arguments, and now I will try to provide some.

In the microvilli of the intestinal epithelial cells, the central actin filaments are connected with each other by the so-called cross-bridges, while similar cross-bridges radiating from the axial filaments connect them with the plasmalemma; this arrangement resembles a spoked 
wheel at cross sections (Mooseker and Tilney, 1975; Matsudaira and Burgess, 1979; Hirokawa and Heuser, 1981; Burgess and Prum, 1982). The cross-bridges may well prevent the swelling of the cell outgrowths. This opinion is supported by evidence from neurons. Tubular outgrowths (neurites) of nervous cells of various vertebrates also have a central axial complex comprising microtubules as well as the actin filaments; this complex is connected with the plasmalemma by cross-bridges (Yamada et al., 1970, 1971; Hirokawa, 1982; Schnapp and Reese, 1982). In the rounded body of the neuron the filaments are arranged irregularly and there are fewer transverse connections (Hirokawa et al., 1984). An even more demonstrative example of the stabilizing function of the cytoskeleton is the concurrent changes in the external appearance and the internal structure of a growing neuron (see Jacobs and Stevens, 1986). The connections between filaments and the plasmalemma do not develop in the neuron body and it remains saclike until the end; young outgrowths whose cytoskeleton is not strictly organised yet have an irregular shape; mature neurites with an axial cytoskeletal complex and cross-bridges look like regular cylinders.

No connections resembling cross-bridges are present in blebs, which have actin filaments only in the cortex. However, such connections are present in lamellipodia, which are rich in actin filaments. Tosney and Wessells (1983) observed filaments stretched between the upper and the lower plasmalemma in the lamellipodia of the neurite growth cone and provided a schematic drawing. In the absence of filaments the upper membrane moved far apart from the lower one and the lamellipodium swelled as a result (Tosney and Wessells, 1983, Fig. 17). Lewis and Bridgman (1992), who also worked with the lamellipodia of the neurite growth cone, found two groups of actin filaments. Long filaments passed along the lower and the upper plasmalemma, while short filaments were located obliquely between the upper and the lower plasmalemma. "The shorter meshwork filaments may act as scaffolding, maintaining the volume of the lamellipodia" (Lewis and Bridgman, 1992, p. 1241). In the lamellipodia of the epidermal cells of the clawed frog (Xenopus), "A fibrillar meshwork connects the 'dorsal' and the 'ventral' cell membranes" (Bereiter-Hahn et al., 1981, Fig. 8, legend). In Fig. 9 the authors showed a lamellipodium with a swelling not far from the edge, noting in the legend that at the edge "... the membranes are held together by a fibrillar meshwork. In the swollen region the filaments are less dense [emphasis mine VB]." In the text they were even more certain: "... formation of a flat cytoplasmic lamella requires interconnection of the membranes by filaments (Fig. 8). Weakening of this interconnection would lead to the formation of bulges or blebs due to internal hydrostatic pressure" (Bereiter-Hahn et al., 1981, p.301; see also Fig. 27a).
To sum up, the cytoskeleton seems to play an important but a passive role in the development of cell outgrowths. Hydrostatic forces combined with the cytoskeleton may give rise to evaginations of any shape.

\section{EMBRYOS AND LARVAE OF VERTEBRATES}

Evaginations of visceral organs - We will consider transformations of visceral cavitary organs using evidence mainly from the gut, which has especially many derivatives: its evaginations give rise to various glands, the swim bladder, lungs etc. An increasing complexity of visceral organs is often considered as the transformation of the epithelium. This approach is perfectly summed up in the following quotation: "The mammalian lung, like many other organs, develops by branching morphogenesis of an epithelium" (Pepicelli et al., 1998, p. 1083). At the same time, the data obtained, incidentally, from studies dealing with the morphogenesis of lungs showed that the inner mass of lungs is actively involved in the morphogenetic process. In experiments of Sorokin (1961), fragments of mammal lung rudiments transferred from the embryo's body into a solution stopped branching. The budding resumed only after the open ends have healed (capped), i.e. after the fragments became closed bodies. In what way were closed fragments different from the open ones? The difference was that they could increase the volume of the inner mass and its pressure on the sheath by secreting the liquid into its cavity. We have to conclude that it was the impact of the inner fluid on the epithelium of the rudiments that underlay their increasing complexity, i.e. the emergence and the growth of the lung buds.

That the inner mass of the gut increases in volume in the course of its natural growth and morphogenesis needs no proof. Expanding, the liquid mass exerts a uniform pressure on the epithelium, displacing it centrifugally (see Fig. 1B). The epithelium which is being displaced encounters the resistance of the outer mass. In case of visceral organs of embryos and larvae, this mass is represented by mesenchyme. Its major component in respect of volume is an amorphous extracellular matrix. The matrix, rich in water, has been referred to as a dilute hyaluronate gel (Newman and Frisch, 1979). This means that the resistance of the outer mass to the sheath of the growing organs should be uniform in all points of its area. The epithelium of the organs cultured in the solution naturally encounters the same resistance.

If both the inner and the outer masses exert a uniform pressure on the sheath of a cavitary body (see Fig. 2A), we should look for the cause of its non-uniform growth (the cause of the emergence of evaginations) in the properties of the sheath. The epithelial sheath is composed of two layers. One consists of cells and the other is represented by the basement membrane. What are the mechanical 
possibilities of these layers? The cellular layer in principle might expand in proportion to the increase of the inner mass by proliferation, providing no considerable resistance. The basement membrane, on the contrary, is an inert structure. It can thicken (does thicken) by addition of new material to the open surface but it cannot expand (increase its area) on its own. In this respect the basement membrane is similar to the cell wall of plants and fungi, whose area increases passively under the stretching impact of the expanding inner mass. Similarly to the cell wall, the inert basement membrane should offer an especially strong resistance to the growing inner mass.

How is the epithelium organised at the sites where gut derivatives develop? It has often been noted in descriptions of the morphogenesis of lungs (Wessells, 1970; Brody et al., 1982; Gallagher, 1986; Moore et al., 2005) and salivary glands of mammals (Grobstein and Cohen, 1965; Nakanishi et al., 1986, 1988) that the basement membrane is thinner at the sites of evaginations than in the neighbouring areas of the epithelium. A similar picture has been observed during branching of capillaries: the basement membrane of vessel walls was destroyed at the branching sites (Patan, 2000). These data indicate that evaginations of the gut and vessels develop according to the laws of the hydromechanical model (Fig. 2B).

One may say that the destruction of the basement membrane sets the cellular layer of the epithelium free to form evaginations by itself. Undoubtedly, epithelial cells participate actively in the development of the derivatives. It is by means of their proliferation that the sheath of new structures grows. Can, however, the epithelial layer evaginate by itself in a certain direction and build regular shapes in the process without the impact of the inner mass? To note, these shapes are not just regular: lung buds and lobules of salivary glands, for instance, are blebby. Such structures are unlikely to emerge without a uniform pressure of the liquid inner mass. To remind, nobody questions the leading role of the hydrostatic pressure in the formation of cell blebs. We should also bear in mind the results of experiments of Sorokin (1961), which clearly indicate the importance of the inner mass for the development of evaginations.

External evaginations - Limb buds are the best known external evaginations of embryos and larvae. Their development has been in the focus of numerous studies, descriptive as well as experimental. In particular, considerable attention has been paid to the stimulating role of the so-called apical ectodermal ridge (AER). The mechanical aspects of the growth and morphogenetic processes are, however, seldom discussed. It is assumed that these processes are determined by the inner mesenchymal mass. For instance, Ede and Low (1969) in a computer modelling study of chick limb bud development took into account only the proliferation and the migration of the mesenchy- mal cells filling it. The authors realised that the ectoderm created the surface tension but did not include it into their model and did not even show it in schematic drawings, depicting the buds as "naked". Interestingly, while the epithelial sheath receives much attention in the studies of evaginations of internal organs of vertebrates (see above), in studies of the development of external evaginations its active mechanical role is not recognised.

Three components are always present in our schemes: the inner mass, the outer mass and the sheath separating the former from the latter (Fig. 2). The sheath of the embryos (the ectoderm) is organised in the same way as that of visceral organs. However, in contrast to the homogenous liquid mass filling the visceral organs, the mass occupying the primary cavity of the embryo has a complex composition. Some of its peripheral components such as myomeres could, in principle, exert a local pressure upon the ectoderm, evaginating it (similarly to actin bundles in the pushing filament hypothesis, see above). However, none of the embryonic or larval organs contact the ectoderm directly, as can be easily seen at various histological sections (Figs. 3D; 4D). Electron micrographs show that not even the cells (or, rather, the cell bodies) closely adjoin the ectoderm. For instance, in the chick limb bud densely filled with mesenchymal cells (see Fig. 4A for an example) only their fine projections reach the basement membrane (Singley and Solursh, 1981); “...there is a region about $0.3 \mu \mathrm{m}$ thick from which the main body of the cells is always excluded" (Gould et al., 1972, p.328). Sometimes there are almost no cells at all in the body outgrowths (Figs. 3A, C; 4B). This may be the case even in the limb buds of tetrapods, which are usually packed with cells. For instance, the distal limb segment (the finlet) of the larvae of the Siberian salamander (Salamandrella) contains few cells but grows very fast (Borkhvardt, 1994, 2000). These examples clearly indicate that the cells do not exert a direct mechanical impact on the ectoderm.

Thus, only the amorphous extracellular matrix adjoins the ectoderm and may exert pressure upon it. This means that the heterogeneous inner mass of the entire embryo or larva interacts with the ectoderm as a common highly pliable mass. Encountering, as it grows, the resistance of the sheath (first of all, of the basement membrane), this mass, similarly to the liquid of the visceral organs, should distribute the pressure uniformly across its entire area, and the body shape should be close to spherical. Indeed, though an embryo (or larva) does not look like a simple regular body, its parts often do. The body and the tail of early embryos may be rounded in cross section, while the amphibian limb buds arise as hemispheres. Smooth curves of the ectoderm, which can often be seen on preparations (Fig. 4D), also produce the impression that the body walls experience a uniform pressure of the inner mass. The outer liquid mass also presses against the embryos and larvae uniformly. Why, then, does their body 

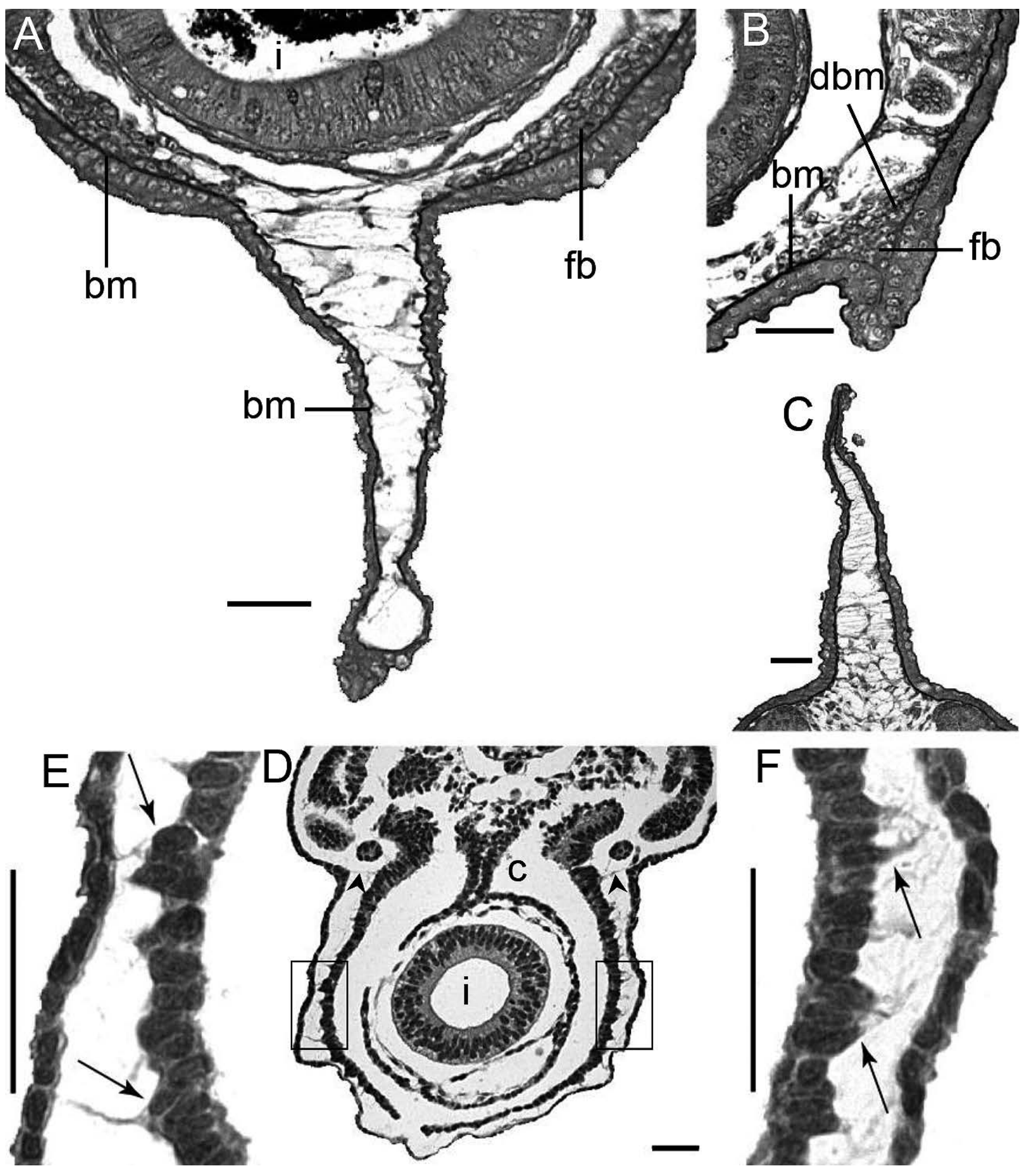

Fig. 3. Basement membrane and linkers in fish embryos and larvae.

A-C: Larvae of beluga, Huso huso, at stages 39 (A) and 40 (B, C) (stages identified after: Schmalhausen, 1975). Cross sections of fin buds (A, $B)$, the ventral (A) and the dorsal (C) unpaired fin fold. A: Basement membrane in the distal part of the unpaired fin fold is thinner than in the proximal part; basement membrane of fin buds differs little in thickness from that of the neighbouring skin areas. B: Basement membrane underlying the ectodermal cell layer of a more developed fin bud is much thinner than that of the neighbouring skin areas. The old basement membrane has detached and can be seen in the deeper region. C: Collagen linkers connect contralateral walls of the fin fold. D: Embryo of spiny dogfish, Squalus acanthias, $16 \mathrm{~mm}$ in length. Transverse section of the posterior part of the trunk. Body walls form longitudinal depressions at the sites where horizontal linkers (arrowheads) connect the walls of the coelomic cavity with the ectoderm. E, F: Fragments enclosed in the boxes in D. The cells of the parietal wall of the coelomic cavity, connected with the ectoderm (arrows), leave the epithelial layer.

bm - basement membrane; c - coelomic cavity; dbm — detached basement membrane; fb — fin bud; i - intestine.Bars — 50 $\mu m$. 

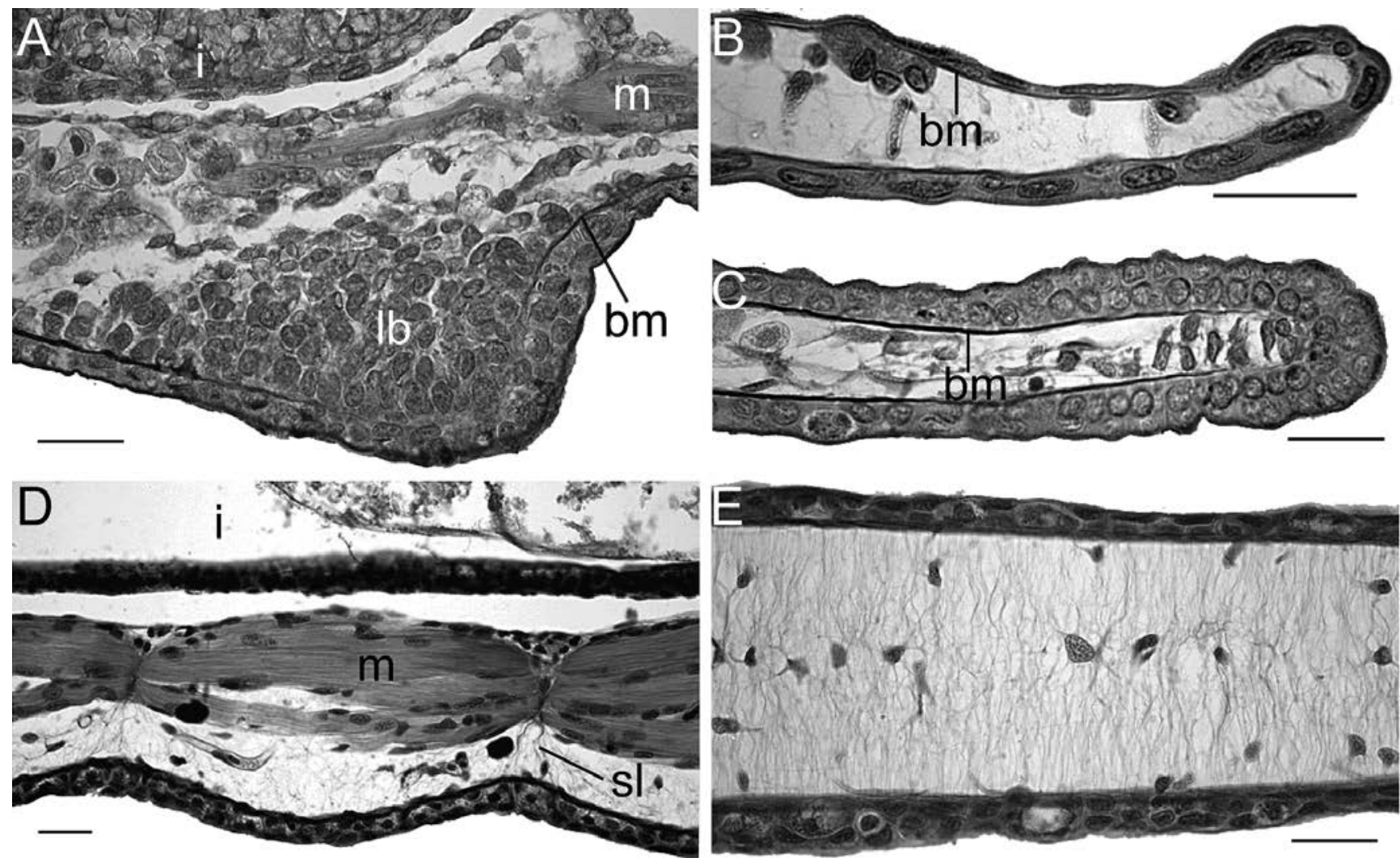

Fig. 4. Basement membrane and linkers in urodele larvae.

A-C: Larvae of Siberian salamander, Salamandrella keyserlingii, at stages 30 (A), 35 (B) and 34 (C) (stages identified after Sytina et al., 1987). Frontal sections across the forelimb bud (A) and the balancer (C), vertical section across the operculum (B). Basement membrane at the apex of these evaginations is almost indiscernible. D, E: Larvae of crested newt, Triturus cristatus, at stage 48 (stages identified after: Glaesner, 1925). Frontal sections in the presacral trunk region (D) and across the ventral fin fold (E). D: Collagen linkers concentrate in the region of transverse myosepta, the body wall forms depressions there. E: Numerous collagen linkers uniformly connect contralateral walls of the fold, these walls are smooth. Cranial ends of sections are on the left.

bm — basement membrane; i — intestine; lb — limb bud; $\mathrm{m}$ - myomere; sl — septal linker. Bars - 50 $\mu \mathrm{m}$.

grow non-uniformly and acquires an irregular shape? Why do some of their parts grow faster (evaginate)? The answer given by the hydromechanical model is as follows: because the sheath (in this case, the ectoderm) is mechanically heterogeneous and its parts resist differently to the pressure of the inner mass. Is that really so?

Various external evaginations of embryos and larvae have a relatively weakly developed basement membrane. This is especially well documented in fin and limb buds, whose basement membrane is always much thinner than in the neighbouring regions of skin. Within the buds, it is especially thin in their distal part and may even be interrupted at the very top, below AER (Balinsky, 1957; Jurand, 1965; Ede et al., 1974; Kelley and Bluemink, 1974; Raynaud et al., 1974; Borkhvardt, 2002b). Salpeter and Singer (1960) observed a thinning of the basement membrane at the tip of regenerating limbs of the newt. In some cases the membrane may be thin because it is underdeveloped (Smith et al., 1975). Usually, however, it seems to be the same as elsewhere at first (Fig. 3A) but later detaches from the ectodermal cell layer and is destroyed. A new base- ment membrane of the fin (limb) bud is much thinner than that of the neighbouring skin areas (Figs. 3B; 4A). It remains like this for a long time: I observed a relatively thin apical membrane in amphibian limbs until the complete formation of the cartilaginous skeleton. In the limbs of urodele larvae the thinning of the basement membrane was found not only in the distal part but also at the rapidly growing pre- and postaxial edges (Borkhvardt, 2001). A relatively thin basement membrane was also found at the tail tip of stellate sturgeon embryos (Borkhvardt, 2002b), at the tip of unpaired fin folds in the larvae of fish (Nadol et al., 1969; Fig. 3A, C) and amphibians, at the tip of the operculum (Fig. 4B) and external gills of amphibian larvae and in urodele balancers (Fig. 4C).

Linkers - Comparing various evaginations in vertebrate embryos and larvae, one can see a certain rule. Evaginations filled with liquid are always cylindrical or blebby, while organs containing mesenchyme may also be flattened (Fig. 3A, C). Stroeva (1950) thought that the flat shape of fin folds in amphibian larvae was 

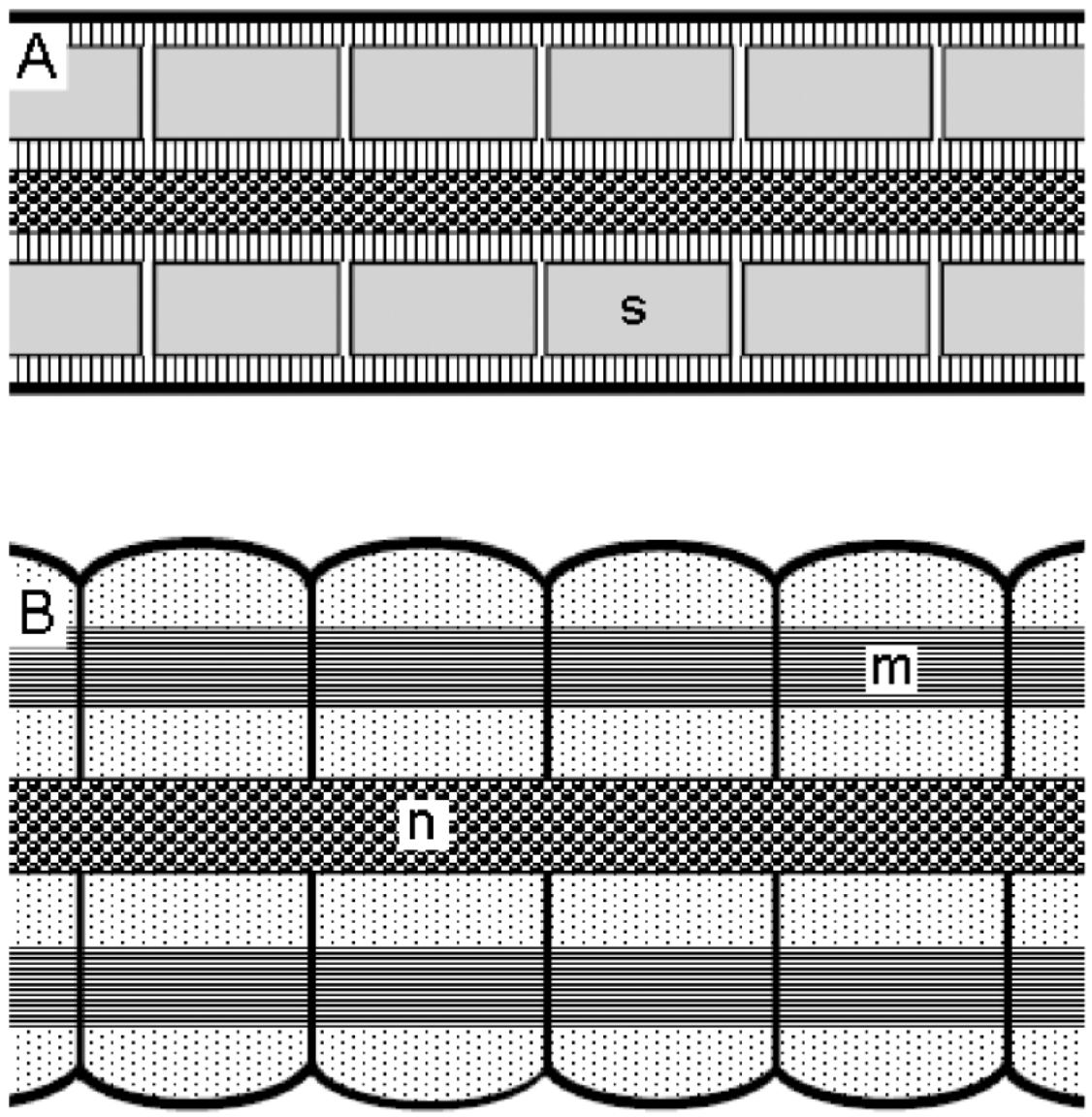

Fig. 5. Reorganization of collagen linkers in early ontogenesis of vertebrates (a scheme). A, B: Frontal sections of the body at successive stages of development. A: Collagen linkers (thin vertical lines) uniformly connect epithelial walls of somites (s) with the ectoderm (thick horizontal lines) and the notochord (n). B: Walls of somites are destroyed, their cells have given rise to mesenchyme (dots). Collagen linkers (thick vertical lines) concentrate in the region of transverse myosepta separating neighbouring myomeres (m). Body walls are wavy because of depressions formed at the sites of the linkers' attachment (the corresponding waviness of the notochord is not shown).

supported by mesenchymal cells, whose outgrowths connected the opposite walls and prevented them from drifting apart. Løvtrup (1983) offered a similar opinion. In general, fin folds contain few cells but many collagen fibres, which link the contralateral walls (Figs. 3C; $4 \mathrm{E})$ and might hamper their diverging. Revealingly, at the anterior end of the dorsal fin fold in toad tadpole, where no such fibres were found, the fold swelled to form a sphere (Borkhvardt, 2000, Fig. 1b); one can see a similar swelling in the distal part of the ventral fin fold of the beluga larva (Fig. 3A). The following example is equally demonstrative. The fibres linking the walls of the unpaired fin fold in newt larvae (Fig. 4E) are arranged densely and regularly, and the walls are smooth. There is no such regularity in paired fins of the larvae of the stellate sturgeon. The walls of their fin folds are adjoined by radial muscles and the bundles of fibres pass only between these muscles, at a large distance from each other. At the sites where the fibres are attached to the walls of the folds, the latter form depressions and the fin surface becomes wavy (Borkhvardt, 2000, Fig. 2b). These facts indicate that collagen fibres linking the opposite walls of fin folds indeed prevent them from diverging, affecting in this way the shape of evaginations. The same thing happens to the whole body of embryos and larvae.

Organs occupying the primary body cavity have no direct contact with the ectoderm (see above) but neither do they "float" freely in the mesenchymal mass. Collagen fibres stretching across the body cavity connect internal organs with each other and with the ectoderm (Lipton and Jacobson, 1974; Bellairs, 1979; Schoenwolf, 1979; Borkhvardt and Kovalenko, 1985, 1986). In this way, organs and fibres connect contralateral body walls. The distribution of the linking fibres changes in the course of the ontogenesis. At the stage of non-segmented mesoderm and whole somites the fibres are arranged regularly (Fig. 5A), and their mechanical action is difficult to reveal at that time (cf. Fig. 4E). After the disintegration 
of somites and the formation of myomeres, the fibres are concentrated (retained) in the area of transverse myosepta, at which time their influence becomes visible. Furrows are formed at the sites of the body wall where fibres are attached, and the surface of embryos and larvae becomes wavy as a result (Figs. 4D; 5B). At the sites where the inner ends of the same septal fibres are attached to the notochord and the neural tube, the latter form respective protrusions (Borkhvardt and Kovalenko, 1985, Fig. 1c, 1986, Fig. 1; protrusions of notochord are not shown in schematic drawing $5 \mathrm{~B}$ ). In shark embryos collagen fibres are also arranged in horizontal "plates", connecting the ectoderm with the outer (parietal) wall of the coelomic cavity; the ectoderm forms longitudinal depressions at the sites of the fibres' attachment (Fig. 3D).

Fibres and cells connecting contralateral walls of cavities and preventing them from diverging have been called linkers (Borkhvardt, 2000). They play, of course, a passive role, which is expressed only in the process of the inner mass growth and the centrifugal movement of the sheath induced by it. The linkers disrupt a smooth expansion of the inner mass, preventing the formation of perfectly rounded bodies. Flattened fin folds of fish (Fig. 3A, C) and amphibians are a good example of that. The gut and its derivatives, blood vessels and kidney tubules are filled not with mesenchyme but with liquid, which lacks collagen fibres. There are no linkers there, and so these bodies are always cylindrical or blebby.

Linkers may be involved not only in the formation of shape but also in some other mechanical processes. For example, it cannot be ruled out that they play an important role in the destruction of epithelial walls of somites, the causes of which are still obscure. As embryos grow, the ectoderm, which is being displaced centrifugally, pulls along the somites attached to it by linkers (see Fig. 5A). Since the contralateral somites are also connected with the help of the central axial organs (notochord in Fig. 5), the emerging forces simply tear the mesodermal blocks apart (Borkhvardt, 2002a). New facts support and expand this idea. It can be seen in Figs. $3 \mathrm{E}$ and $3 \mathrm{~F}$ that it is the cells of the parietal coelomic wall attached to the ectoderm that leave the epithelial layer; it seems that the centrifugally moving ectoderm literally tears them out of it.

As we have seen above, cell outgrowths may have filaments linking in some way contralateral areas of the sheath (the plasmalemma). Several authors (Bereiter-Hahn et al., 1981; Lewis and Bridgman, 1992) thought that these filaments were responsible for the flattened shape of lamellipodia. I have extended this idea to cylindrical outgrowths in which radial cross-bridges connect the plasmalemma with the bundle of central longitudinal filaments. The similarity of all these structures with collagen linkers is evident. In the light of this, the phenomenon of linkers seems to be universal.

\section{CONCLUSION}

Hydromechanical model describing the development of evaginations in walled cells can be applied to evaginations of other living cavitary bodies (Fig. 2B).

\section{Invaginations}

\section{ANIMALS}

"Invagination of epithelial tissue occurs during gastrulation, neurulation, and organogenesis in many organisms. However, the underlying morphogenetic mechanisms of invagination are not understood." (Kimberly and Hardin, 1998, p. 235). Similarly to the authors of these words, most scientists studying invagination in animals focus on the epithelial layer, and invagination is usually regarded as the bending of this layer. Observations of epithelium fragments taken from the embryo's body are considered as an important method of studying invaginations. In the nature, however, epithelium never exists in fragments. An epithelium is always a sheath of a cavitary body. It does not just bend during invagination: it sinks. And it does not just sink: it immerses into a closed cavity filled with an incompressible substance. How can it do so? This is a pertinent question for all cavitary bodies, both multicellular and unicellular, and every invagination model should provide an answer.

Odell et al. (1981) are the only authors I know who did ask this question and answered it too. Discussing gastrulation in the sea urchin, they decided that the invagination (primary gut, archenteron) itself eliminates the resistance of the inner mass, by pushing the liquid out of the blastula mechanically. The liquid indeed leaves the blastula cavity during gastrulation in various animals. There is demonstrative evidence from amphibians and, especially, the lancelet, whose embryo by the end of gastrulation becomes a two-layered cup almost devoid of any cavity. But can organisms (their invaginations) push out the liquid? To remember a vivid expression of Carlson (1983), a figure similar to cup-shape gastrula of the lancelet can be obtained by pressing a punctured rubber ball with a finger. Living cavitary bodies, however, are not punctured balls. The permeability of the blastoderm in the sea urchin, for example, becomes very low by the time of gastrulation (Moore and Burt, 1939). The removal of water along the osmotic gradient is a more comprehensible and feasible way to decrease the inner mass of cavitary bodies. As we know, this is something that single cells can easily do.

As early as in the XIX ${ }^{\text {th }}$ century an opinion was suggested that invagination of the blastoderm during gastrulation occurred because of the lowering of pressure inside the blastula (see Rhumbler, 1902). This idea is attractive. If the pressure in the cavity drops down below the level of the outside pressure, the outer mass would 
automatically tend to move into the low pressure region. If it succeeds in overcoming the resistance of the sheath, it would start to move together with it towards the centre of the cavity. In this scheme, the decrease in the volume of the inner mass is not the consequence of the invagination, as suggested by Odell et al. (1981), but, on the contrary, its cause. The question about the penetration of the sheath into an incompressible mass does not arise at all, and the solution to the problem presents itself.

Discussing the hypothesis of the pressure difference, Rhumbler (1902) noted that it was quite usual for the blastocoel to become smaller during gastrulation but made several critical remarks (I will refer to one of them below). At the same time, he paid special attention to the transformations of cells in the invaginating part of the blastoderm (Entodermplatte of the German authors). It was this route that the scientific thought was to follow.

Gastrulation in echinoderms - Gastrulation in echinoderms is probably the best studied invagination process in animals. Nevertheless, Davidson et al. (1999, p. 221) had good grounds to say that "... despite a century of work... there is still no answer to the question: How do sea urchins invaginate?"

In echinoderms, gastrulation starts with the bending of the so-called vegetal plate, the part of the blastoderm composed by high, columnar cells. Moore and Burt (1939), who cultured the plate in vitro, showed that it could invaginate even after its dissection from the embryo. After these results were corroborated (Ettensohn, 1984), few doubts were left that “...the forces responsible for invagination must reside in the vegetal plate itself" (Davidson et al., 1995, p. 2005).

It is usually thought that the buckling of the vegetal plate is caused by the narrowing of the apical (outer) ends of its cells (see Davidson et al., 1995, 1999; Nakajima and Burke, 1996; Kimberly and Hardin, 1998). As their ends narrow, these cells become pyriform (they are also referred to as bottle cells or wedge-shaped cells), and the entire plate looks like a fan opened inside at sections. The apical parts of the cells are thought to be compressed by means of contraction of cortical actomyosin rings. It is considered possible (see Davidson, 2012) that the actomyosin contraction involves an entire cellular sheet rather than separate cells but the result is the same: the vegetal plate buckles. To remind, it is not at all evident that the actomyosin of non-muscle cells can contract. In this case, too, it has been left without special proof.

The buckling of the vegetal plate is just the beginning of gastrulation. After that the primordial archenteron elongates. This process cannot be explained by cell contraction. Some other mechanism must be at work. The researchers divided the gastrulation process into two stages to be studied separately. Describing the changes occurring at the second stage, Dan and Okazaki
(1956) hypothesised that the archenteron elongated by means of the pulling efforts of the filopodia of secondary mesenchymal cells connecting the apex of the primary gut with the blastoderm of the animal pole. This idea was criticised (Hardin and Cheng, 1986; Hardin, 1988) on the grounds that the archenteron elongated successfully even if the filopodia were initially absent or artificially removed. It was also suggested that the growth of the archenteron was associated with the changing shape (elongation along the longitudinal axis and flattening) and the rearrangement of cells composing the primary gut wall (Ettensohn, 1985a; Hardin, 1988, 1989). The causes of the cell transformations were not discussed. Kominami and Takata (2004) noted that the rearrangement of cells occurred not in all sea urchins.

The hypotheses do not address the question of how the archenteron overcomes the resistance of the inner mass of the blastula (with the exception of Odell et al., 1981, see above) and do not take into account the state of the blastocoel. In many animals the blastocoel diminishes during gastrulation (this has long been known see Rhumbler, 1902). What about echinoderms? The authors working before the 1930s mostly thought that the sea urchin embryos did not enlarge in the course of gastrulation, only Hörstadius was of a different opinion (see Dan and Okazaki, 1956). Ettensohn (1984) observed an enlargement of the embryos but in another study (Ettensohn, 1985a) noted that their volume did not change. Dan and Okazaki (1956) also mentioned that the volume remained the same. A constant total size (blastocoel plus archenteron) indicates clearly that the blastula cavity (primary body cavity) decreases in size as the archenteron grows.

Kominami and Takata (2004, text and Fig. 2) recorded that the embryos enlarged only at the second stage of gastrulation, while at the stage of primary invagination their size remained more or less stable. At the same time, these authors found periodic changes in the turgor pressure of the embryos. It was rather high at the stage of blastula and dropped before the gastrulation. By the end of the first stage of gastrulation the internal pressure increased again, and archenteron stopped to grow for about two hours; then the pressure decreased somewhat, and the primary gut elongated fast. An artificial increase of the pressure inside the blastula hampered the invagination of the vegetal plate.

Kominami and Takata (2004) were familiar with the old idea about the leading role of the low internal pressure in invagination. It would seem that their own data might have made them sympathetic about it but this was not so. They admitted that a decrease in the turgor pressure during normal development facilitated the bending (invagination) of the vegetal plate and wrote elsewhere that a "...change in the level of osmotic pressure of blastocoel would be necessary for the progress of gastrula- 
tion" (Takata and Kominami, 2001, p. 1097). However, they did not go any further being sure that the "pressure difference" hypothesis had been refuted by the results of experiments on the culturing of the excised vegetal plate. Let us give a closer consideration to these results.

Moore and Burt (1939) excised the vegetal plate at the stage of the early gastrula already after the start of the invagination. They attached importance to the fact that the early archenteron did not regress but continued to grow. In general, it can be seen in their Fig. 1 that the invagination increased little after the extirpation of the vegetal plate. The main changes occurred at the plate edges, which expanded and met to restore a closed sphere in which the archenteron grew further. The authors did not discuss how and by what means it grew. Ettensohn (1984), contrary to Moore and Burt (1939), excised the vegetal plate before the gastrulation. He also observed the formation of the archenteron. However, it is unclear from the text when the primary gut was formed: before or after the closure of the cavity. The only photograph showing the archenteron (Fig. 2) was made at the stage when the embryo has already restored the shape of a closed cavitary body. An indisputable conclusion one can make from the experiments of Moore and Burt (1939) and Ettensohn (1984) is that the vegetal plate excised from the embryo can grow and close into a ball. Its ability to give rise to the archenteron before the transformation into a closed cavitary body is yet to be confirmed. Let us allow the possibility, however, that an excised vegetal plate in the experiment does bend and that the arising "invagination" does grow. Even if this were so, this would not solve the problem of gastrulation since normally the archenteron develops under entirely different conditions, having to incorporate itself into an incompressible mass.

Moore and Burt (1939) did not only observe an excised vegetal plate. They also cultured whole sea urchin embryos, placing them, in particular, into hypertonic solution. Removing the water osmotically from the early blastula, they induced an invagination of its wall. The result was a structure they called a pseudo-gastrula. If the water was removed from the late blastula, the normal gastrulation process accelerated. These results clearly testified in favour of the pressure difference hypothesis but neither the authors nor the readers arrived at this conclusion. I think that both facts and general considerations (see the beginning of this section) agree with an old idea that the cause of the invagination of the blastoderm is the decrease of the pressure inside the blastula below the level of the outside pressure.

An argument in favour of the idea that it is the pressure of the outer liquid mass that determines the formation and the growth of the archenteron may be found in observations on the exogastrulation process made by Dan and Okazaki (1956). These authors managed to pro- voke the formation of the exogastrula at the stage when the archenteron was rather long. It shortened, and the epithelium surrounding the blastopore evaginated. Finally, the archenteron, in the words of Dan and Okazaki (1956), was pushed out of the blastocoel. This process is reminiscent of the eversion of fingers of a rubber glove, which many of us must have observed in everyday life. To turn out the fingers, one fills the glove with air, closes the opening (creating a closed cavity) and presses the inflated part. The fingers pop out with surprising speed. The mechanism of the eversion is clear in this case (the inner air mass pushes the fingers out) and is likely to be the same in case of the archenteron everting during exogastrulation. But if the archenteron can be pushed out by the inner liquid, it can also be pushed in by the outer liquid. The only condition for this is that the internal pressure should drop below the external one.

If the blastoderm is indeed invaginated ("pushed in") by the outer liquid, why does this happen in the vegetal plate area? Both the inner and the outer liquid, naturally, exert a uniform pressure upon the entire blastoderm. This means that the answer should be looked for in the properties of the plate. Rhumbler (1902) was quite right in thinking that if the invagination occurs under the pressure of the outer mass, it should occur at the site where the blastoderm offers the least resistance to it. On the contrary, an invaginating Entodermplatte, according to Rhumbler, should resist even more than the other parts of the blastoderm since it is thicker. This was one of the reasons why he was sceptical about the entire pressure difference hypothesis.

The vegetal plate of the sea urchin blastula, which is composed of columnar cells, is also thick but has soft spots. For instance, the basement membrane lining the inner surface of the blastoderm is absent on the walls of the invagination (Ettensohn, 1984). Moreover, there is one more structural feature in this region that promotes to a local weakening of the blastoderm. It is flat in the area of invagination. This feature is reflected in the name "vegetal plate" (as well as in the German term Entodermplatte applied to blastulae of various animals). The role of arches in supporting architectural construction is well-known. It is clear that a flattened blastoderm would not resist the external pressure as successfully as an arched one. The flattening of the blastoderm, in its turn, may be a consequence of the changes in the shape of cells, which become columnar at a certain site.

According to the new model (or, rather, a revitalised old one) (Fig. 2C), the gastrulation of the sea urchin does not need to be divided into various stages, and this process is considerably "simplified" as a result. The pressure of the outer liquid is quite sufficient to ensure the development of the archenteron from the bending of the vegetal plate to the point when the primary gut reaches the animal pole. Later the archenteron forms a new 
evagination, paired or unpaired: the coelomic pouch. Its formation can be easily explained, again, by the pressure of the outer liquid filling the primary gut. Of course, the growth of the archenteron and its derivatives proceeds to a great extent by proliferation of epithelial cells. The pressure of the outer mass first of all directs their growth and gives them a certain shape though some "forced" invagination of the blastoderm portions neighbouring the vegetal plate is also possible.

In modern models of invaginational gastrulation especial importance is attached to the transformation of cells of the buckling blastoderm - they become bottle-shaped. This occurs not only in echinoderms but also in other animals such as the lancelet (see Rhumbler, 1902) and amphibians (see Keller et al., 2003; Lee and Harland, 2007). It is thought that the cells change shape actively by contraction of the actomyosin cortex in the apical part. Their transformation results, in turn, in the bending (invagination) of the blastoderm. As regards echinoderms, I have accepted another hypothesis: the development of the archenteron occurs under the pressure of the outer mass. If so, how can we explain the change in the shape of the vegetal plate cells?

To begin with, wedge-shaped cells can be found not only at the invagination site. As early as in 1902 Rhumbler noted that in various animals all cells of the blastoderm were wedge-shaped, with the narrowed parts facing the blastocoel. Does that mean that the cells actively contract their inner parts? Nobody suggests that. What, then, determines the shape of these cells? The answer, I think, is simple. The outer and the inner parts of the cells of the spherically bent blastoderm experience different conditions: the former lie in the area with a greater curvature and the latter, in the area of a smaller curvature. Under such conditions densely packed cells would inevitably broaden towards the outer space and narrow towards the inner space. The wedge shape of the cells is especially conspicuous in a thick blastoderm, where the outer and the inner surface differ considerably in length. A good example is the blastoderm of the actinia Nematostella vectensis, which is composed by very tall cells (see Kraus, Technau, 2006).

When the blastoderm invaginates, the relationship is inversed. Now the inner part of the cells lies in the area of a greater curvature, while the outer part lies in the area of a smaller curvature. Predictably, the cells invert their shape, becoming thin in the apical part and thick in the basal part. It does not matter what force has bent, e.g., the vegetal plate. It could be the pressure of the outer mass, in which case the change in the shape of the cells would be merely a side effect of the invagination.

In conclusion, I would like to propose an idea, which is not directly related to gastrulation. It was born under the impression from the discovery of Kominami and Takata (2004). They found that the turgor pressure in the sea urchin embryos changed periodically. We know that periodic changes of the volume and, thus, of the turgor pressure are characteristics of cells. Trinkaus (1969) thought that rhythmic changes of the volume (pulsation) were the usual state of animal cells. It is possible that periodic changes of the volume and the inner pressure (to a great extent determined by influx and efflux of water) are the usual state of all turgid cavitary bodies, both unicellular and multicellular.

External invaginations in embryos and larvae of vertebrates - The rudiments of the central nervous system, the internal ear, olfactory organs and the eye lens are formed by invagination into the primary body cavity of early embryos. The rudiments of skin glands of tetrapods are formed in the same way but much later. Though the processes of development of the neural tube and sensory organs are studied quite well, there is no consensus concerning the driving factors (cf. Burnside 1973; Schroeder, 1973; Zwaan and Hendrix, 1973; Karfunkel, 1974; Schoenwolf, 1979; Brun and Garson, 1983; Ettensohn, 1985b; Schoenwolf and Smith, 1990). It was not long ago that the author of a general review (Gordon, 1985, p. 229) announced that "Neurulation is an unsolved process." The most popular opinion is that the epithelial layer buckles due to changes in the shape of its cells, which become pyriform, with a narrow apex and a broad base. The transformation of cells, in its turn, is the result of contraction of submembrane actomyosin rings in the apical part (Bernfield et al., 1973; Burnside, 1973; Schroeder, 1973; Spooner, 1973; Odell et al., 1981). We have already come across this hypothesis when discussing gastrulation. Now it is time to add some further critical remarks to what has already been said.

It would seem that the apical parts of cells squeezed by contractile rings should be rounded at cross-section, and the cells are indeed shown like this at schematic drawings (e.g., Burnside, 1973, Fig. 15). However, Schroeder (1973) did not find any regular shapes in histological cross-sections of cells of an invaginating chick neural plate. All submembrane filaments were organised into polygonal figures, and the cells themselves were also polygonal at the sections (Schroeder, 1973, Fig. 8). The author was surprised by this picture but failed to find a satisfactory explanation. Ybot-Gonzalez and Copp (1999, p. 273) came to the conclusion that "...contraction of actin microfilaments is not obligatory for epithelial bending during embryonic morphogenesis." According to Colas and Schoenwolf (2001), the apical contractile rings stabilise the shape of cells rather than change it. Finally, it would do no harm to remind that the ability of the cell cortex to contract is yet to be proven.

We know the conditions experienced by the sheath (the ectoderm) of embryos and larvae. It is washed by liquid from the outside. The inner mass contacting the 
ectoderm is at first a liquid too and later a semifluid extracellular matrix. Thus, the ectoderm experiences a uniform pressure both from the outside and from the inside. Could we apply the hydromechanical model in this case too? We have a difficulty here. As concerns invaginations, the model makes a provision that the internal pressure is lower than the external one (Fig. 2C). However, after gastrulation embryos and larvae grow, and this may only happen when the internal pressure is higher than the external one. On the other hand, it is difficult to understand how the epithelium may sink into the primary cavity filled with an incompressible substance (a liquid or an extracellular matrix) without resorting to the factor of a low internal pressure. The opinion of Odell et al. (1981) that invaginations push the excess of inner mass outside (see above) seems even less probable. I just cannot imagine the rudiment of, say, internal ear or the lens pushing out anything through the basement membrane.

What is the solution of this problem? We will find it if we accept that the development of embryos is not a continuous but an oscillating process, during which growth alternates with periods of "stagnation" or even negative growth. Examples of such processes in the living nature are growth fluctuations of pollen tubes and fungal hyphae, pulsation of animal cells and changes in the pressure inside the forming gastrula of sea urchin. A similar process can be observed in the early ontogenesis of vertebrates; it is indicated by the reduction in the body volume in embryos and larvae. The first example of this process is a noticeable decrease in the cross-section of the caudal region in embryos and larvae of fish and amphibians; this fact is even used in tables of normal development (see Dabagyan and Sleptsova, 1975; Ginsburg and Dettlaff, 1975). The second example is a decrease in the size of the entire body during metamorphosis of amphibian larvae. Its parts may also become smaller in the process as illustrated by the notochord of the fire-bellied toad (Anura) in the trunk region (Borkhvardt, 1982). It is during metamorphosis that the epidermis parts giving rise to skin glands begin to invaginate. So, there is a distinct temporal correlation of the two processes: a decrease of the body cavity and the invaginations of the integument.

Another part of the hydromechanical model, the local weakening of the sheath, is more certain. The basement membrane is very thin in the rudiments of olfactory sacs of Ambystoma (Balinsky, 1957) and on the walls of the apical portions (oval buds) of mammary ducts growing into the body of mice embryos (Williams and Daniel, 1983). During formation of skin glands in amphibians, the basement membrane is being destroyed at the sites where epidermis invaginates (my own observations). The second structural change weakening the resistance of the epithelium to the pressure is also familiar to us. The neural tube, olfactory sacs, auditory vesicles and lenses in all vertebrates are formed, similarly to the archenteron of the sea urchin, as invaginations of flat parts of the epithelium composed by one layer of columnar cells. This is reflected in the names of these parts. The rudiment of the neural tube is called the neural plate, while the rudiments of other organs are called placodes. The term "placode" originates from a Greek word meaning "plane" or "plate". We have already discussed that the flat parts of the sheath should offer a lesser resistance to the pressure.

Finally, the very appearance of the ectodermal invaginations suggests that they develop under a uniform pressure (of the outer liquid): these invaginations are rounded. It is no coincidence that the rudiment of, for instance, the internal ear is called auditory vesicle.

Ingrowth of the derivatives of visceral organs into the body cavity of vertebrate embryos and larvae Earlier in this paper we considered the derivatives of the gut and other visceral organs as evaginations. These evaginations, similarly to the invaginations just discussed, immerse into an incompressible mass filling the primary body cavity. This means that they encounter the same difficulties as invaginations. Therefore, we will pay them some attention in this section, too.

Discussing the immersion of the blastoderm and the ectoderm into an incompressible mass, I have discarded the possibility that the invagination mechanically pushes some of the inner mass out of the primary body cavity. Theoretically, the invagination can make its way using another method. It can transmit the pressure onto the sheath through the inner mass, displacing the sheath centrifugally. In this way, an invagination at one site would be compensated by an evagination at other site or sites. The entire body would enlarge in the process, which does happen during embryonic and larval development. However, speaking of invagination of the ectoderm, it is very difficult to imagine that the epithelium of invaginating rudiments of, let us say, auditory vesicles can generate a force capable of displacing the sheath of the entire embryo. Evaginations of visceral cavitary organs can generate such a force since these organs can build up the "omnipotent" hydrostatic pressure by secreting liquid into its closed cavity. Plant cells, in which this pressure reaches many atmospheres, are a good example. When visceral organs enlarge (which they do by producing evaginations among other things), they may easily displace the ectoderm of the embryos and larvae off the centre.

The blood system, which is closed throughout its existence, is the most easily understandable internal cavitary organ in this respect. The first elements of the kidney, the pronephric tubules, are connected with the coelom and also form a closed cavitary body together 
with it. The pronephric duct formed on the basis of the first tubules is in essence an evagination of the coelomic cavity, initially elongating as its blind outgrowth. Later the renal ducts open to the outside but they do not remain open permanently.

The development of the gut and its derivatives is more complicated. We will consider it using mostly evidence from amphibian gut. Until the end of gastrulation the gut (the archenteron) opens into the environment. The difference between the internal and the external pressure determining the growth of the archenteron is due to the drop of pressure into the blastula cavity (see above). The situation changes after the closure of the blastopore. Secreting liquid into its cavity, a closed gut can increase its internal pressure above the pressure in the body cavity. Overcoming the resistance of the surrounding mass and (together with the other growing parts of the organism) the resistance of the ectoderm, the gut grows and produces various evaginations. Soon, however, the situation changes one more time: the mouth and the anus open, and the gut together with its derivatives becomes an open system once again. After this, the development of gut derivatives may slow down as indicated, it would seem, by the data of Mashkovtsev (1935), who compared the development of lungs in the larvae of various amphibians. In the larvae of the axolotl and the frog the lungs are connected with the environment through the glottis. The animals begin to breath atmospheric oxygen while still in the water, as they rise to the surface and swallow air. In toad tadpoles the glottis closes early and the lungs, loosing connection with the environment, become closed bodies; similarly to all the other early gut derivatives, they are filled with liquid. The lungs of toad larvae undergo a much more intensive increase in complexity than those of the axolotl and the frog larvae.

Mashkovtsev (1935) thought that the development of lungs in tadpoles of the toad occurred under the stretching influence of the lung fluid on their sheath. This seems reasonable since the pressure of the fluid inside closed lung rudiments can easily reach high values exceeding the pressure in the body cavity. The lungs of the axolotl and the frog, according to Mashkovtsev, also develop under the stretching influence, in this case, the influence of air. This conclusion was based on the results of experiments in which the larvae were deprived of the possibility to swallow air; the lungs of such animals showed almost no increase in complexity. It is not entirely clear, however, how the air pressure in the open lungs of larvae may exceed the pressure in the body cavity. In principle, the pressure difference may arise as a result of a decrease in the pressure inside the cavity. This scenario is exemplified by the familiar mode of air breathing in amniotes. The pressure in their body (or pleural) cavity drops when the so-called suction pump is at work (when the movement of ribs and, in mammals, of the diaphragm expands the thoracic cavity). Amphibians have no suction mechanism. Conditions favourable for the action of the pressure difference factor are created during metamorphosis, when the larvae become smaller and the pressure in their body cavity decreases correspondingly. It is possible that the major changes occur in the open lungs at that time (Mashkovtsev [1935] did not indicate when they occurred). Besides, it cannot be ruled out that small fluctuations of pressure in the body cavity (analogous to RVI and RVD of cells) occur throughout the larval lifetime.

\section{CELLS}

Endocytosis - Small invaginations (vesicles) are constantly arising on various cells. The inner surface of the vesicles may be coated with clathrin proteins, and it has been suggested that this layer may play an active role in invagination (Heuser, 1989; Pypaert et al., 1991; Qualmann, Mellor, 2003). Observations on clathrin lattices of broken-open cells show that they can indeed bend in the presence of ATP (Heuser, 1989). However, endocytic invaginations are not always coated with clathrin. An important role in the organisation of endocytosis is also thought to be played by actin filaments. The involvement of these filaments is judged upon merely by their presence in the zone of invaginations and by the cells' response to treatment with cytochalasins and latrunculins. These methods might indicate a possible involvement of actin filaments but cannot identify their role. Indeed, this role is still not deciphered (see Engqvist-Goldstein and Drubin, 2003; Yarar et al., 2005; Kaksonen et al., 2006). There is a hypothesis (see Kaksonen et al., 2006) based on the assumption that actin filaments are attached to the cell membrane: as new monomeres are added to the outer ends, the filaments move inwards and pull the membrane along with them. Grębecki (1991) and Kłopocka et al. (1996) thought that the contracting cortex pulled the plasmalemma inside during formation of pinocytotic channels in the amoeba. However, spermatozoa of Ascaris have neither actin nor myosin filaments but numerous depressions arise on their body just the same (see Sepsenwol et al., 1989).

Researchers studying endocytic invaginations ignore the general question of how the sheath (the plasmalemma) sinks into a closed cavity filled with an incompressible substance. In this case, however, factors preventing invagination are given some attention. For instance, Raucher and Sheetz (1999) noted that a bending membrane had to overcome its own tension. Sometimes, when factors hampering endocytosis in walled cells are discussed, turgor pressure comes up. Oparka et al. (1990) did not observe any endocytic vesicles in turgor cells of onion epidermis. Gradmann and Robin- 
son (1989) calculated that clathrin-coated vesicles could form in plant cells only if the internal pressure was less than one atmosphere.

In principle, the problem of the immersion of the plasmalemma into the cell has quite an easy solution. It is the same solution that has just been suggested to explain the invagination mechanism in multicellular cavitary bodies (Fig. 2C). Its applicability to cells can be supported with some experimental data. Invagination in cells can be easily triggered artificially by placing them into hypertonic solution, which osmotically drains water out of them. (To remind: Moore and Burt [1939] performed similar experiments on sea urchin embryos.) In this medium, animal cells and protists decrease in size, and depressions appear on their surface (Mast and Hopkins, 1941; Bereiter-Hahn and Strohmeier, 1987). Dai et al. (1998) let the neurons of Lymnaea (Gastropoda) swell in hypotonic medium and then transferred them into isotonic medium; the cells shrank, and deep invaginations were formed on their surface facing the substrate. I would like to emphasise that invaginations appeared on the flat cell surface (Dai et al., 1998, Fig. 2; see also Morris et al., 1997, Fig. 1b). Bleb-like invaginations were observed in striated muscle fibres shrinking in hypertonic solution (Dydyńska and Wilkie, 1963).

Invagination during lowered internal pressure (Fig. 2C) seems just as evident as evagination during increased internal pressure (Fig. 2B). This is indeed supported by experimental results but is this so under natural conditions? For the present, I can give only one example, which concerns changes occurring with Acanthamoeba during encystment (see Bowers and Korn, 1969). At first the amoeba became rounded, then it started to lose water and to diminish in size; at the same time, the cell membrane invaginated in various sites and micro-cracks opening to the outside arose at the cell body.

Let us assume that the pressure of the outer mass is indeed the driving force of endocytosis, as it is during invagination of the epithelium. This mass is either a liquid or a semifluid matrix. Its pressure would tend to create spherical shapes. In many instances endocytic invaginations are indeed spherical as evidenced by the fact that they are described and depicted as vesicles. It is difficult to imagine that such regular shapes are created by, let us say, pulling efforts of actin filaments (see above).

Since the outer mass is highly pliable, its pressure is distributed uniformly across the entire cell surface. In such a case, the location of invaginations should be determined by the mechanical heterogeneity of the sheath or, in other words, by the location of weak spots. Numerous facts indicate that this is so in case of evaginations of various bodies and invaginations of the epithelium. Information about endocytic vesicles is at present much more scarce. I know only one kind of cell invaginations, whose sheath is patently thinner than the neighbouring areas: the so-called surface vesicles in resting smooth muscle cells (Devine et al., 1972, text and Figs. 9; 22). Besides, I would like to remind that in the experiments of Dai et al. (1998) and Morris et al. (1997) invaginations arose on flat cell surface, which indeed should be the first to yield to the pressure of the outer mass.

Endocytosis occurs throughout the lifetime of the cell. So does the antagonistic process, exocytosis. Zonia and Munnik (2008, p. 861), who observed tobacco pollen tubes, noted that "Hypotonic treatment and cell swelling stimulated exocytosis and attenuated endocytosis, while hypertonic treatment and cell shrinking stimulated endocytosis and inhibited exocytosis." What are "swelling" and "shrinking"? They are phases of pulsation, which, as we know, is a normal state of the cell. This means that pulsation is the basis on which endocytosis and exocytosis, vital processes in the life of cells, are performed by turns.

Cell division - In this section I will speak about division, mostly the division of unwalled cells, which is, in essence, a kind of invagination. There is an almost perfect agreement among researchers regarding the forces driving this process. It is thought that the cell is constricted and divided by a contractile ring composed of actin and myosin filaments. The idea of the contractile ring was suggested quite a long time ago (Marsland and Landau, 1954). It gained a universal recognition (see Sanger and Sanger, 2000; Yumura, 2001; Pollard, 2003; Albertson et al., 2005) after actin and myosin filaments were found under the plasmalemma of the cleavage furrow.

What are the arguments in favour of the contractile ring idea except the presence of actin and myosin filaments in it? The connection between the ring and the division seems to be indicated by the fact that the ring appears by the beginning of the division process and disappears after its completion. Further, cells treated with cytochalasin do not divide (though there are data indicating they may do so - Krishan, 1972). Undoubtedly, the belief in the contractile abilities of actomyosin in the cortex of non-muscle cells weighs heavy on scholarly minds. This belief, in turn, is nourished by evidence from muscle contraction. Schroeder (1990, p. 81) wrote in a review paper: "From the description thus far, there is little reason to doubt that the contractile ring is an actin-myosin complex that functions, at least superficially, like a muscle." In muscles or, more precisely, in striated muscle fibres, actin and myosin filaments are arranged in strictly regular sarcomere lattices, and their sliding was directly observed. There are reports that the arrangement of actin and myosin filaments in the contractile ring resembles that in the sarcomere (Sanger and Sanger, 1980, 2000) but more numerous observations indicate the contrary. Fishkind and Wang (1993), having made a special study on dividing rat kidney cells, con- 
cluded (p. 846) that in the contractile ring "...the actin scaffolding of dividing cells is composed of a complex three-dimensional network of filaments, rather than a simple circumferential ring (Fig. 9)." Fig. 3g in Mabuchi et al. (1988) also shows a network of actin filaments, which are, in addition, connected with each other by bridges. I fail to understand how in such a state they can slide against myosin filaments (which are, incidentally, not shown). Indeed, sliding of the filaments in the contractile ring has never been observed.

The contractile ring concept runs into further difficulties. Schroeder (1990) even made a list of them. I will cite here just one of his questions. How can the contraction of the submembrane actomyosin complex produce unilateral (e.g., in coelenterates) or discoidal (e.g., in birds) cleavage? The mechanism of a contractile arc has been suggested to explain unilateral cleavage (Salmon and Wolniak, 1990) but its action has never been explained. Sanger and Sanger (2000) thought that a complete ring was necessary for the cells to cleave into two daughter cells. As far as discoidal cleavage is concerned, there are no suggestions that I know of.

The most important argument reinforcing a sceptical outlook on the contractile ring model is that cells can divide without myosin. Mutant Dictyostelium cells that lack myosin may form deep and broad pseudo-cleavage furrows (Fukui et al., 1990) or even complete the division (Knecht and Loomis, 1987; Neujahr et al., 1997; Zang et al., 1997). The same behaviour was observed in rat kidney cells and fibrosarcoma cells treated with blebbistatin, a myosin inhibitor (Kanada et al., 2005). The authors considered this behaviour as direct evidence in favour of "...contractile ring-independent equatorial furrowing in mammalian cells" (Kanada et al., 2005, p.3865). Gram-negative bacteria, which have no wall, divide by furrowing similarly to animal cells (Koch, 1990). However, they have neither actin nor myosin. The tubulin ring of gram-negative bacteria is thought to be a functional analogue of the contractile ring of eukaryotic cells (Lutkenhaus and Addinall, 1997) but the mechanism of its action is obscure. To remind, the main idea underlying the muscle contraction theory is the interaction between filaments of two various types.

In a historical review of the 30 -year-old actomyosin contractile ring concept Uyeda and Nagasaki (2004, p. 4) were bold enough to say that "...there is surprisingly little compelling evidence in the literature that myosin II, or the purse-string mechanism, is essential for cytokinesis of adherent somatic cells. Evidence taken to support the essential role of myosin II in cytokinesis is mostly ambiguous, if not negative." It turns out that actin ring filaments are not indispensible for division either. Schroeder (1972) observed the sea urchin zygote to complete cleavage (its diameter in the furrow area ranging from $2.5 \mu \mathrm{m}$ to zero) without any ring whatsoever.
In the end of this critical review, we should recall another familiar fact, which is generally ignored by scientists. Not only cells divide but also their organelles such as mitochondria, Golgi apparatus, vacuoles of walled cells and plastids of plant cells. Their division is not necessarily associated with cytokinesis. The Golgi apparatus, for instance, is constantly forming secretory vesicles. No contractile ring has ever been observed in any organelle.

The ability of cells to divide without an actomyosin ring does not mean, of course, that it is uninvolved in cytokinesis when present (and it is present in most cells). However, the above examples clearly indicate the existence of a different mechanism (or mechanisms) of division of cavitary bodies. If so, the traditional scheme should be supported by an especially solid argumentation. Among other things, it has to explain how the plasmalemma sinks into a closed cavity filled with an incompressible substance (any invaginational model should explain that). Schroeder (1990) did not take this difficulty into account but had a poor opinion of the ring's ability to divide the cell all the same. "By electron microscopy, the contractile ring is really extremely thin. At magnifications low enough to see the outline of the cell and its furrow, the contractile ring is an almost invisibly thin layer beneath the membrane; at first glance, it seems almost too thin to be the effective motor of furrowing" (Schroeder, 1990, p. 80).

In principle, the problem of immersion into an incompressible cytosol could be solved by the hydromechanical model. Are there, apart from general considerations, any concrete arguments in its favour?

When discussing invaginations, I said that a drop in the internal pressure of a cavitary body was the first condition of the hydromechanical model. I know two instances of such a change in case of cell division. Firstly, Wordeman and Cande (1990, Fig. 1) published a series of micrographs illustrating the division of a diatom alga by furrowing, which is unusual for plant cells. It can be clearly seen in the micrographs that immediately after division the "sum" of the two daughter cells was smaller than the mother cell. This means that the division was accompanied by a decrease in the volume and, thus, a decrease in the internal pressure. The second example is the behaviour of plant cells in the course of plasmolysis. When a plant cell is placed in a weak hypertonic solution, the protoplast decreases in volume, separates from the cell wall and becomes spherical or ellipsoid; such protoplast may divide by furrowing (Komarov, 1949, Fig. 32). Since the volume of the protoplast and, thus, its internal pressure decrease before the start of cytokinesis, there are good reasons to suppose that the division is caused by the drop in the pressure inside the protoplast.

While these facts agree with the hydromechanical model, observations on the division of unwalled 



Fig. 6. Two patterns of cell pulsation (a scheme).

Top row: "Simple" pulsation. The cell increases and decreases alternatively, its shape does not change. Bottom row: "Complex" pulsation resulting in cell division. A: A restraining ("contractile") ring (black vertical band) appears at the cell equator. B: Cytoplasmic volume (internal pressure) increases; the restraining ring prevents centrifugal displacement of the sheath in the middle part of the cell; unrestrained poles move apart; the cell becomes ellipsoid. C: Internal pressure drops below the external one; the outer mass invaginates in the equator area where the sheath has the least curvature. D: Internal pressure grows, the cell elongates, its thickness in the zone of the cleavage furrow (in the zone of the restraining ring) remains the same. E: Internal pressure drops, the cell continues to divide.

cells seem to disagree with it. An increase rather than a decrease in the cell size can be often seen at schematic drawings illustrating the process of their division (e.g., Hiramoto, 1981, Fig. 1). Judging from micrographs made at the middle and the final stages of division (Neujahr et al., 1997, Fig. 8B), rapidly dividing Dictyostelium cells did not change in length and decreased in volume, while slowly dividing cells became distinctly longer (Fig. 8C, full series). In general, cell elongation during division is a common phenomenon. I have noted it myself on videos showing cell division. Koch (1990) even suggested a mechanism of division of gram-negative bacteria implying that the diverging poles "tear" the cell apart.

Any increase, be it in volume or in linear size, is incompatible with the hydromechanical model because according to it invagination is caused by a decrease in the internal pressure. However, this difficulty is easily removed if we assume that the change in the volume of the inner mass (the internal pressure) of dividing cells is an oscillating process: the mass (the pressure) now decreases now increases. This is a fairly realistic assumption. Rhythmic changes of the volume (pulsation) are a usual feature in the life of cells, as we have already mentioned more than once. Here is another such fact from the life of unwalled cells. Dan and Dan (1947) described a cleaving medusan egg at three stages of the first cleavage and noted: "When the blastomeres round up again in later stages, the contour becomes smaller once more." (p. 171; emphasis mine - VB).

If the internal pressure of a cleaving cell fluctuates, the state of the furrow may be expected to do so too. Its deepening during the drop of the internal pressure should be followed by a return to the previous state after its rise. However, for cytokinesis to be completed, there should be no recurrent movements of the furrow floor or they should be smaller than the advance. This is where the (passive) influence of the "contractile" ring may come into play. It girdles the cell like a hoop, preventing it from increasing in diameter (Fig. 6, bottom row). The poles, which experience no such restraining influence, diverge under the pressure of the swelling cytoplasm. It is clear that a limiting rather than a contractile ring may well act without myosin filaments: actin filaments alone are quite sufficient for a passive restraint. Actin filaments are always present in rings of eukaryotic cells. In bacteria, the restraining role can be played by the tubulin ring.

The ring is likely to start playing its passive morphogenetic role even before the formation of the cleavage furrow. This is due to the fact that in the anaphase, before the division, the cell becomes ellipsoidal even it was spherical before (see Fig. 6B). This change might be considered as the initial phase of division occurring by means of the contractile action of the ring. However, another scenario is also possible: when the cytoplasmic volume increases, the cell poles diverge under its pressure; the cell elongates but does not thicken due to the restraining influence of the ring. A similar phenomenon can be observed during non-uniform growth of plant cells elongating at the opposing poles: the cellulose microfibrils of the cell wall girdling the cell in its middle part prevent the cell from thickening but do not prevent the turgor pressure from diverging the poles (see Harold, 1990; Baluška et al., 2003).

The second condition of the hydromechanical mod$\mathrm{el}$ is the weakness of the sheath at the site of evagination (Fig. 2B) or invagination (Fig. 2C). During formation of outgrowths of unwalled cells their sheath weakens due to the destruction of the cortex or the weakening of its association with the cell membrane. This does not seem to be the case with dividing cells; moreover, the presence of 
the "contractile" ring might even enhance the resistance of the sheath to the pressure of the outer mass. However, another weakening factor may act here. As I have just said, a cell about to divide is close to an ellipsoid and therefore is less convex in the middle than at the poles. This is where the cleavage furrow arises. Revealingly, before the start of cleavage, an equally cleaving zygote of the sea urchin flattens in the zone of the future furrow uniformly along the entire equator (Mabuchi, 1994, text and Fig. 2d) while a non-equally cleaving zygote of the clawed frog (Xenopus) flattens only from above (Bluemink and de Laat, 1973, text and Fig. 1a).

In a cleaving zygote of Xenopus new membrane areas are incorporated into the plasmalemma of the furrow (Bluemink and de Laat, 1973; Byers and Armstrong, 1986; Danilchik et al., 2003). This is generally characteristic of cleaving animal cells: “...targeted membrane addition during cleavage furrow formation is a fundamental and widely conserved mechanism of animal cytokinesis" (Albertson et al., 2005, p.92). Byers and Armstrong (1986) thought that new material is added to the plasmalemma in the region of the side walls of the cleavage furrow. Danilchik et al. (2003) found numerous exocytotic pores at the furrow floor and decided that it was there that the new material was incorporated. The addition of new material to the plasmalemma increases its expansion ability, which should also diminish the membrane resistance to the pressure of the outer liquid.

To sum up, according to the proposed model the division of the cells is based on its usual pulsatile activity: alternating decrease and increase of the volume by means of efflux and influx of water (Fig. 6, top row). The presence of the hoop-like ring disrupts the simple oscillation process, which results in a directional change in the shape of the cell: 1) its elongation and 2) formation of a median invagination (Fig. 6, bottom row).

\section{CONCLUSION}

The cause of both invagination and evagination is the difference in the pressure inside a cavitary body and outside it. "Invagination of a homogenous outer mass in the zone of a weak sheath" (Fig. 2C), in essence, equals "evagination of a homogeneous inner mass in the zone of a weak sheath" (Fig. 2B) but, as it were, with an opposite sign. The scope of the hydromechanical model is expanded.

\section{Cell locomotion}

Movement of cells upon the substrate (crawling) is often considered as a sum (a sequence) of operations, and the movement of the anterior end is studied separately from that of the posterior one. We have also resorted to this approach discussing the evagination of the leading edge of moving cells earlier in this paper. Let us now focus on the movement of the cell body and tail, keeping an eye on the entire cell in the process. Movement of these parts is less often discussed in the literature while judgments are even more diverse. However, the diversity of opinions has not resulted in clarity of understanding, and lamentations are frequent. I will cite Fukui et al. (1999, p. 877) as an example: "... little is known about the mechanism that brings the posterior cell body forward." On my part, I must confess that the hypotheses suggested in this field of research were especially difficult to understand, let alone analyse.

Bretscher (1988) compared the cell to a tank. Based on experiments with the use of membrane markers, Anderson et al. (1996, p. 1209) decided that "...ellipsoidal cell body [of keratocytes - VB] rotates during translocation," the cell body apparently "rolling" after the lamellipodium. Iwadate and Yumura (2008) stated the presence of an "elastic transition zone" connecting the anterior and the posterior ends of the cell and apparently responsible for the pulling up of the posterior edge during evagination of the leading one. The authors did not explain how this zone was organised.

The movement of the cell is often thought to be associated with the contractile actions of actomyosin complexes. There is no consensus as to how and where this happens. Lo et al. (2004, p. 982) wrote about the role of myosin in the locomotor activity of the cell: "Although myosin II is known to play an important role in cell migration little is known about its specific functions." Munevar et al. (2001, p. 1755) were sure that the forces determining the movement of fibroblasts "....are generated predominantly by actin-myosin-II-based contractions..." but "Where the forces are generated and how the contractions are regulated remains unclear." Here is another observation concerning the involvement of contractile complexes into cell locomotion: "Myosin II is believed to play an important role in organizing protrusive activity and traction forces in migrating cells, but precisely how it produces its effects on cell locomotion remains unclear" (Kolega, 2006, p. 4435). Svitkina et al. (1997), who studied locomotion of keratocytes, thought that the cell body moved by contraction of an actomyosin complex situated in the transition zone between lamellipodia and the cell body. I fail to grasp how this contraction can move the cell body. Wittmann and Waterman-Storer (2001) noted that contraction occurred in the cell body but gave no further explanations. Contractile actions were also thought to be associated with the posteriormost cell region (Jay et al., 1995; Iwadate and Yumura, 2008).

The fact that myosin-mutant Dictyostelium amoebae crawl more slowly than wild-type cells is considered as a weighty argument in favour of the connection between locomotion and actomyosin contraction (Jay et al., 1995; Iwadate and Yumura, 2008). Slow movement, 
however, is quite different from no movement at all and I, contrary to the authors, interpret these data as an indication that cells may move without myosin, that is, without actomyosin contraction. Other observations are even more demonstrative. For instance, neutrophils treated with myosin inhibitor (2,3-butanedione monoxime) slowed down the retraction of the tail region but this occurred only "...on adhesive substrates... and not on poorly adhesive substrates..." (Eddy et al., 2000, p. 1287). Fibroblasts treated with another myosin inhibitor, blebbistatin, even increased the rate of locomotion (Even-Ram et al., 2007) and so did epithelial kidney cells with artificially deformed myosin filaments (Zurek et al., 1990). Lo et al. (2004, p. 988) were surprised to find that "...myosin IIB null fibroblasts were not only capable of migration but also migrated at a higher raw speed than did control cells". These examples show that cells can move quite successfully without the participation of actomyosin complexes.

Spermatozoa of nematodes demonstrate especially clearly that cells may crawl without actomyosin (see Nelson at al., 1982; Roberts, Streitmatter, 1984). They have almost no actin and no myosin, and it is thought that during locomotion these proteins are functionally substituted by filaments composed of a specific "major sperm protein" (MSP). In the anterior part of the cell these filaments are arranged in bundles and form gel. It was suggested that "...elastic energy stored during bundle formation generates tension in the cytoskeleton to pull the cell body forward when the gel solates" (Bottino et al., 2002, p.375). I find the model more comprehensible in another interpretation (see Roberts and Stewart, 2000; Miao et al., 2003). According to in vitro observations, the fibres composed of MSP may both elongate and shorten; when they shorten, particles anchored at their ends move along with them. The authors hypothesised that the shortening of these fibres at the base of the pseudopodium results in the forward movement of the cell body. They did not specify to which parts of the cell body the filaments were attached or how their efforts were transmitted.

Amoeba proteus, which has excellent locomotion skills, is a traditional model object in studies of cell migration. It is time that we discuss how this amoeba moves.

A. proteus has at least two characteristics important in the context of our topic. Firstly, its endoplasm lacks actin filaments capable of pushing (evaginating) the apical plasmalemma during cell locomotion or growth of pseudopodia. According to the general opinion, this role is played by the cytosol. In other words, in this case we know exactly how the mechanical effort is transmitted onto the cell membrane and evaginates it. Secondly, a powerful flow of the endoplasm is observed when an amoeba moves or forms pseudopodia. It is directed from the posterior edge (the uroid) to the leading edge or to the tip of a pseudopodium. Parts of the tail sheath get inside the cell by intensive endocytosis (pinocytosis). The flow of endoplasm transports this material to the evaginating edges where it is used for the construction of new sheath areas (Stockem and Kłopocka, 1988). This means that the cytoplasmic flow plays the key role in locomotion and pseudopodial growth, exercising the mechanical impact on the plasmalemma and delivering the building material. Correspondingly, researchers who studied locomotion of the amoeba "...concentrated mainly on the mechanisms of generating and controlling the endoplasm stream" (Grębecki, 1984, p. 116).

At first sight, an amoeba seems to divulge its secrets readily by parading its inner life. This "transparency", however, is illusory. According to Allen (1961, p. 212), "There are very few biological problems in which so many theories have been proposed to explain so few data, as has been the case of ameboid movement." Thirty years later Dembo (1989, p. 1053) confessed that the "Debate about the mechanism of amoeboid motions has gone on since the last century..., but as yet the dynamical laws that govern these phenomena are largely unknown."

In a classical study, Mast (1926) gave the following synopsis of the mechanism of amoeboid locomotion. A resting amoeba is in the state of turgor. Its “... internal osmotic pressure counterbalances the elastic tension of the plasmagel [a dense peripheral layer of the cytoplasm], and the beginning of movement in such a specimen is due to local solution and consequent weakening in the plasmagel, resulting, owing to contraction elsewhere [and the subsequent increasing internal pressure], in local stretching and the formation of a protuberance" (Mast, 1926, p.410). Further, “...locomotion [of amoeba] may be looked upon as being essentially due to contraction of the thick sheet [of plasmagel] covering the posterior end, forcing the column of fluid, the plasmasol, in the [plasmagel] tube forward against the thin sheet [of plasmagel] at the anterior end, stretching it and pushing it forward together with the surface membrane..." (Mast, 1926, p. 412; comments in square brackets and emphasis mine - VB). The plasmasol (the endoplasm) flows forwards, thickens and builds new parts of the tube of the plasmagel (ectoplasm). The new parts of the tube do not move relatively a point in space. The tail, which is moving forward, approaches and incorporates them. The incorporated ectoplasm thins and flows forward again.

The scheme proposed by Mast - uniform internal pressure plus a local weakening of the sheath - is actually the hydromechanical model, which we have been discussing from the beginning. This scheme, with additions and updates, remains relevant to this day. It has been suggested, for instance, that the weakening of the 
evaginating apical sheath occurs as a result of the destruction of the cortex or its detachment from the plasmalemma (Stockem, Kłopocka, 1988; Grębecki, 1990). We also know now that the cortex of a moving amoeba is periodically destroyed not only at the leading edge but also at the posterior end (Stockem and Kłopocka, 1988). However, the nature of the cytoplasmic flow largely remains a mystery. Most researchers, following Mast, think that the cytosol moves forward owing to the contraction of the cortical layer of the tail. The contraction of the uroid in a moving amoeba seems to be indicated by the wrinkles on its surface (Mast, 1926; Wehland et al., 1979). The forward movement of the posterior cell end has also been explained by the contraction of the tail. However, Grębecka and Grębecki (1975) did not consider this explanation satisfactory and hypothesised that "...uroid, undergoing its own contraction, is simultaneously dragged forwards by the contraction in the trunk" (p. 359). The belief that contraction is associated with the uroid seems to be mostly based on the conviction that it is the only way to explain the forward flow of endoplasm from the tail.

The above ideas concerning locomotion in the amoeba raise several questions. The first question is traditional: how does endocytosis occur in the tail, i.e. how does the invaginating plasmalemma overcome the resistance of an incompressible cytosol? Even a resting amoeba is turgid while during contraction of the cortex the internal pressure should increase further. The second question is: how does the contraction of the tail cortex agree with its periodic destruction? Finally, the greatest doubt: does the contraction of the tail cortex indeed cause the endoplasmic flow?

Why do we see a flow? Because the cytoplasm is moving faster than the entire cell, faster than its tail, and faster than its leading edge. This should not be so according to Mast's model. The contracting uroid pushes a column of cytoplasm (Mast himself wrote it was a "column of fluid"). The front end of the column abuts the plasmalemma and moves together with it, i.e. it should be immobile relatively to it. The plasmalemma of the tail also abuts the endoplasm directly and should also move together with it. In general, in a closed space liquid cannot flow in one direction, it can only circulate. There is an opinion that the circulation indeed occurs: while the central part of the cytoplasm (the endoplasm) is flowing forward, its peripheral layer (the ectoplasm) is moving backwards. This phenomenon is referred to as the fountain streaming (Stockem and Kłopocka, 1988). I said "opinion", not observation, and I mean it. If the forward movement of the endoplasm looks like a powerful flow, the backward movement of the ectoplasm can be revealed, at best, by special methods registering the movement of individual particles (see, e.g., Grębecki, 1984). Mast (1926) observed a reverse movement of the ectoplasm only in a resting amoeba but emphasised that the ectoplasm of a crawling amoeba does not move relatively to a point in space. I watched videos showing locomotion in various amoebae and never saw anything even remotely resembling the reverse movement of the outer layer. Moreover, one really should not expect that from an amoeba moving by contraction of the tail. How can liquid flow into the zone of higher pressure if the contraction of the uroid actually pushes it from there?

Rogers et al. (2008) have recently confirmed the circulation of the cytoplasm in pseudopodia (lobopodia) of A.proteus and named the cause of the reverse movement. "Particles embedded in the endoplasm are forced by the cytoplasmic pressure in the direction of the lobopod, whereas particles embedded in the cortex tend to have a small velocity in the opposite direction. This wellknown but counterintuitive phenomenon, known as the fountain effect... is due to the cortex of the lobopod being connected directly to the cortex of the entire cell: since the cortex is contracting everywhere, it draws the lobopodial cortex back toward the cell body" (Rogers et al., 2008, p. 3317). The total volume of cortical particles moving away from the tip of the pseudopodium was much smaller than the volume of dense inclusions aiming for the tip; the moving peripheral layer was not even uninterrupted (Rogers et al., 2008, Fig. 6a). Besides, cortical particles moved much more slowly. In other words, the direct (endoplasmic) flow was much more powerful than the reverse (ectoplasmic) flow. The returning cytoplasm should of course have a smaller mass than that flowing to the leading edge since some of it is used for the construction of a new tip. Nevertheless, the remains of the erstwhile powerful forward flow are unlikely to be so feeble as to require special detection techniques.

Rogers et al. (2008, Fig. 6a) demonstrated the reverse movement of particles only in lobopodia. If the movement of the peripheral cytoplasm of a projection is produced by the cortical contraction "everywhere", how does the cortex behave in the body of an amoeba? Where does it move there? How exactly does it contract to pull the cortex of the pseudopodium? The authors leave these questions without answers. Let us imagine, however, that some of the solid material does return backwards, to the uroid. Where, in this case, does the "extra" liquid go (I mean the liquid which has not become part of the new evagination)? This extra liquid largely makes up the mass of the endoplasmic flow. Is it also pulled backwards by the contracting cortex?

In conclusion of this review, I would like to point yet again to a remarkable easiness with which various authors operate the idea about the contraction of cortex (cortical actomyosin). In doing so, they build their hypotheses on an idea which is in itself a mere hypothesis without any solid proof (speaking frankly, without any proof at all). 


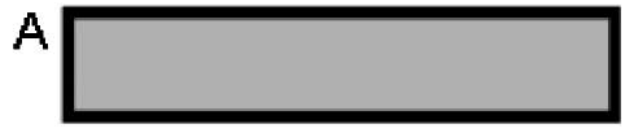

B

\section{$+$}

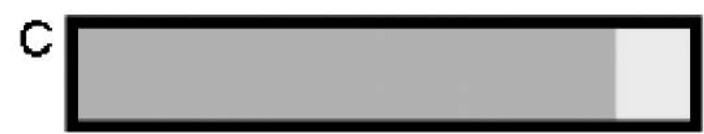

D
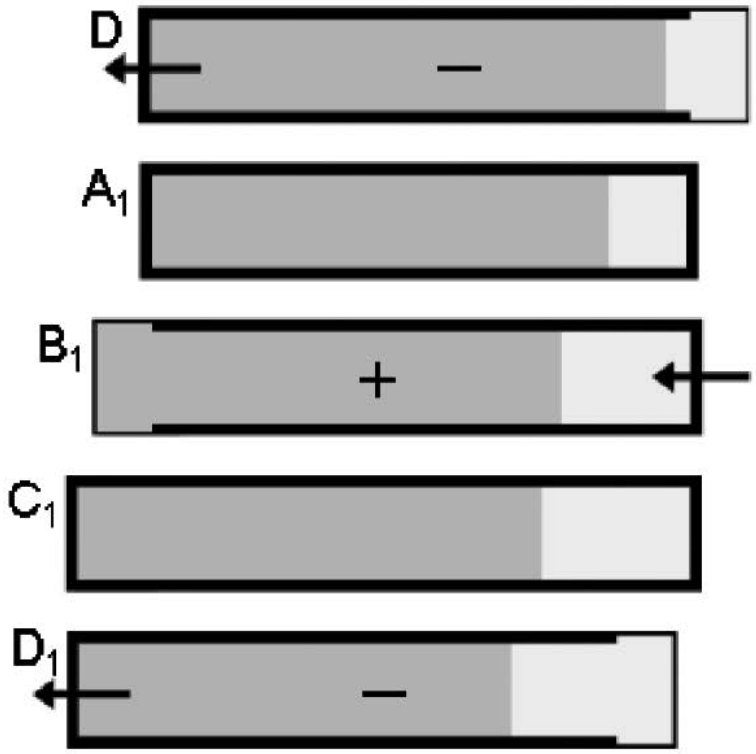

$\mathrm{A}_{2}$

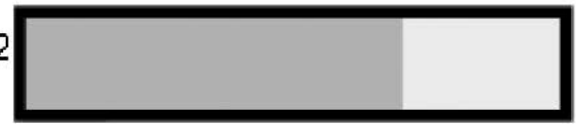

Fig. 7. The running water model of locomotion in amoebae (a scheme). A- $A_{2}$ : Alternating stages of the locomotor cycle. $A, A_{1}, A_{2}, C, C_{1}$ : the entire sheath of the turgid cell offers a uniform resistance to the cytoplasmic pressure (see Fig. 2A). B, $B_{1}$ : water enters the cell in its posterior part (arrow) increasing the cytoplasmic volume (by the amount corresponding to the light grey zone); internal pressure grows (+); the sheath in the anterior part of the cell weakens (thin line), the cytoplasm evaginates there, the cell elongates. $C_{1} C_{1}$ : the sheath becomes whole again, the cell length is maximal. $D_{1} D_{1}$ : water leaves the cell at the leading end (arrow); cytoplasmic volume decreases, internal pressure drops below the external one (-); the sheath in the posterior part of the cell weakens (thin line), the external water squeezes the uroid ("invaginates"), the cell shortens. Elongation of the light grey zone indicates the cytoplasmic stream.

So how does an amoeba crawl, after all? I agree that in order to find an answer one has to solve the enigma of the endoplasmic streaming. The solution, as I see it, is as follows: there is an influx of water in the tail region of the amoeba and an efflux of water at the leading edge. We know that cells can actively regulate their volume by influx and efflux of water. The amoeba can do that too (see Mast and Hopkins, 1941). My hypothesis is based on the only assumption that the amoeba transports wa- ter across the membrane only in certain areas. If this assumption is correct, the locomotor cycle of an amoeba can be represented as follows (Fig. 7).

In the locomotor cycle, dynamic phases (Fig. 7B, $\mathrm{B}_{1}$; $\mathrm{D}, \mathrm{D}_{1}$ ) alternate with resting phases (Fig. $7 \mathrm{~A}, \mathrm{~A}_{1}, \mathrm{~A}_{2} ; \mathrm{C}$, $\mathrm{C}_{1}$; see also Fig. 2A). In the first dynamic phase (Fig. 7B, $B_{1}$ ) water enters the cell at the posterior end and the volume of the cytoplasm increases, increasing the internal pressure. At the same time, the cortex of the leading edge is destroyed or detaches from the cell membrane, the endoplasm pushes the weakened apical sheath (the plasmalemma) forward and moves (evaginates) along with it: the cell elongates. The endoplasm in this phase flows away from the posterior edge but is immobile relative to the leading edge. After that, the influx of water stops and the apical sheath (the plasmalemma plus the cortex) becomes whole again; the amoeba has the maximal length (Fig. 7C, $\mathrm{C}_{1}$ ). In the second dynamic phase (Fig. 7D, $\mathrm{D}_{1}$ ) the water leaves the cell at the leading edge and the internal pressure drops below the pressure outside. The cortex of the uroid is destroyed, the sheath weakens at this site and the outside water forces (invaginates) the sheath of the tail: the amoeba shortens. The endoplasm in this phase flows towards the leading edge but is immobile relatively the posterior end. When the efflux of water stops, the amoeba returns to the initial state (Fig. $7 \mathrm{~A}_{1}, \mathrm{~A}_{2}$ ). The summary displacement of the endoplasm in the course of two dynamic phases (Fig. 7B $+\mathrm{D}, \mathrm{B}_{1}+\mathrm{D}_{1}$ ) is greater than the displacement of the entire cell, i.e. endoplasm moves faster than the cell (which is what the observers see). This model has been referred to as the "running water" model (see Borkhvardt, 2009).

Solving the enigma of the endoplasmic flow, the "running water" model also shows: 1) how the internal pressure increases without any cortical contraction; 2) how it drops, causing endocytosis in the tail; 3 ) how the leading edge and the posterior end of the cell move. According to this model, a moving amoeba periodically changes its size (length). Size fluctuations are indeed found in A.proteus (Satoh et al., 1985). The model also sheds a new light on the causes of wrinkles on the uroid of a moving amoeba. It used to be considered as the result of cortical contraction (Mast, 1926; Wehland et al., 1979), which is mere surmise. On the other hand, we know that cells shrink when they lose water, e.g., in hypertonic solution (see Mast and Hopkins, 1941; Bereiter-Hahn and Strohmeier, 1987; Dai et al., 1998). We also know that the cortex of the uroid is periodically destroyed, i.e. its sheath periodically weakens. When the internal pressure drops, it is the tail plasmalemma that should be the first to be deformed ("wrinkled") by the pressure of water from the outside. Therefore, the depressions at the tail end of a moving amoeba or at the tip of a retracting pseudopodium (Cameron et al., 2007) may be considered as invaginations arising under the pressure of the outer liquid. 
Besides well-visible depressions, the uroid surface of a moving amoeba bears microscopic endocytic (pinocytotic) depressions. They are also thought to be associated with the contractile actions of the cortex (Grębecki, 1991; Kłopocka et al., 1996; partly also Stockem and Kłopocka, 1988). However, they can be just as easily considered as invaginations arising under the pressure of the outside water. Similar microcracks arise on the body of Acanthamoeba when it loses water during encystment (see above). Thus, it would seem that all invaginations of the uroid or retracting pseudopodia in the amoeba are basically similar structures, which differ in size only. Together, these invaginations move the posterior sheath inside, i.e. forward. Locomotion of an amoeba is actually the evagination of the leading edge (under pressure of the cytosol) and the invagination of the posterior end (under pressure of the outside fluid). It might be even better to put it this way: at the anterior end the amoeba propels itself (evaginates), while at the posterior end it is propelled by the outer (invaginating) liquid.

Amoeboid locomotion is characteristic of another popular research object, Dictyostelium. Its unicellular form is referred to as the myxamoeba. We have discussed several times already the experiments of Yoshida and Inouye (2001), who observed formation of outgrowths by Dictyostelium cells. Now it is time to continue the story about the achievements of these authors. In the course of formation of a cylindrical projection the cell body diminished and became impossible to distinguish from the projection; after that the cell started locomotion. Yoshida and Inouye (2001) thought that the body became smaller because of the cortical contraction. However, in another experiment they treated myxamoeba with cytochalasin, which, as we know, blocks the formation of actin filaments. When filamentous actin was lacking, the projection became blebby. While it was enlarging, the cell body was diminishing and finally as good as disappeared: it seemed to "pour" itself into the projection (series of micrographs in Fig. 11). The cortex of the cell body could not contract without actin filaments. Therefore, we have to assume that the body was squeezed by the outer liquid; if so, its internal pressure had to drop below the external one. Later Yoshida and Soldati (2006) seem to arrive at precisely this conclusion. Discussing the mechanism of movement in the myxamoeba, they wrote: "The decrease in cytoplasmic pressure allows the opposite [posterior - VB] end of the cell to retract resulting in net centroid translocation" (Fig. 8, legend). This viewpoint is in conflict with the conditions necessary for the evagination of the anterior end: its increase should be associated with the rising internal pressure. Theoretically we know the solution: the pressure inside the cell should periodically increase (then its projection increases) and decrease (then the cell body decreases). This is exactly what Dictyostelium cells do. Langridge and Kay (2006), who observed blebbing in myxamoebae, noted that the cell volume was "approximately constant" in the process. It can be seen at their graph (Fig. 3) that the volume was oscillating weakly. These oscillations were, of course, caused by efflux and influx of water.

Observations of Yoshida and Inouye (2001) leave me in no doubt of the fact that the posterior part of the Dictyostelium cell is squeezed (moved) by the pressure of the outer liquid. In this respect, locomotion of Dictyostelium is similar to that of A. proteus as described by the "running water" model. Can this model be applied to animal cells? I see no objections to it. The factor of water is becoming increasingly popular in studies concerning locomotion of animal cells. In a paper entitled "Cells move when ions and water flow" Schwab et al. (2007, p. 428) wrote: "... work from the last $10-15$ years provides compelling evidence that ion channels and aquaporins are indispensable for efficient cell migration." Loitto et al. (2002), though accepting the hypothesis about the leading role of actin filaments in the formation of evaginations (see also Loitto et al., 2007), thought that “...water-selective aquaporin channels regulate lamellipodium formation and neutrophil motility" (p. 212). An opinion that the functioning of aquaporins increases the morphogenetic and locomotor activity of cells is based on experimental data. The above conclusion of Loitto et al. (2002) was supported by the results of experiments on blocking aquaporins in neutrophils. According to Hara-Chikuma and Verkman (2006), the cells of kidney epithelium of mutant aquaporin-deficient mice formed fewer lamellipodia at the leading edge than the cells of normal mice and migrated half less actively; artificial incorporation of aquaporins into the membrane of mutant cells corrected these migration defects. The authors emphasised that these morphogenetic and migration differences between the normal and the mutant cells were associated with a different intensity of the transmembrane water transport rather than with the differences in the actin apparatus. Astroglial cells behaved in a similar manner (Saadoun et al., 2005); moreover, the authors suggested that "...the ability of water channels to accelerate cell migration may be a general phenomenon in mammalian cells" (p. 5696). Schneider et al. (2000) and Schwab (2001) assigned the cytoskeleton a leading role in locomotion but thought that the posterior part of the moving cell retracted by means of its contraction after the water efflux.

I would like to offer several other arguments indicating the applicability of the running water model to animal cells. 1) Animal cells pulsate, that is, easily change their volume by osmotic influx and efflux of water. An increase in the volume is a centrifugal displacement of the sheath under pressure of the inner mass, while a decrease in the volume is a centripetal displacement of the sheath under pressure of the outer mass. So, a centripetal 
displacement of the sheath under pressure of the outer mass is a usual feature in the life activity of cells. 2) The cells can weaken the sheath locally by destructing the cortical layer or by detaching it from the cell membrane; earlier I have supported the idea that these operations underlie the development of various evaginations and invaginations. 3) Numerous small and very small invaginations arise at the posterior end (but not at the leading edge!) of a moving amoeba and "destroy" the tail; similar invaginations were described at the apex of decreasing projections of Dictyostelium (Merkel et al., 2000). They were also observed in animal cells, e.g., on collapsing blebs (Cunningham, 1995; Charras et al., 2006). What is especially important in the context of our discussion is that pits were described on the retracting tail of fibroblasts (Chen, 1981, Fig. 9 C-E). It is often thought that all these depressions are due to cortical contraction but it is never explained how it should contract to achieve this. To remind, numerous pits also arise on the body (but not on the pseudopodium!) of crawling spermatozoa of nematodes lacking the actomyosin complex. This example was given in Endocytosis section, where I suggested that small invaginations develop because of a decreasing intracellular pressure (see Fig. 2C). 4) The "running water" model assumes an alternating change in the cell volume and in the cell length. An alternating elongation and shortening was observed in moving endothelial cells (Kolega, 2006).

The leading edge of the cells is constantly built on during locomotion, which means that new materials should be constantly transported there. It is generally recognised that in the amoeba all substances are transported to the apex with the cytosol flow. Building material is also transported to the apex of crawling (and growing) animal cells (see Bray, 1970; Bergmann et al., 1983; Bretscher, 1983; Pfenninger and Johnson, 1983; DeBiasio et al., 1988; Keller and Eggli, 1998; Charras et al., 2006; Fackler and Grosse, 2008) but no cytoplasmic flow is observed in them. How are substances transported there?

There is an opinion that various particles, “...as large as nuclei and as small as RNA molecules" (Pollard, 2003, p. 741), are transported in the cell with the help of molecular motors, which move actively along microtubules (dyneins and kinesins) or along actin filaments (non-muscle myosins) (Vale, 1999; Reese and Haimo, 2000; Mehta, 2001; Gross et al., 2002; Schliwa and Woehlke, 2003; Hirokawa and Takemura, 2005; Hollenbeck and Saxto, 2005; Müller et al., 2008). However, the cytosol also appears to be involved in the transport of various materials. The amoeba is not alone in this respect.

Food vacuoles move with the flow of endoplasm in ciliates. Harold (2002) admitted the possibility that transport inside walled cells might be ensured by flows of fluid. Lew (2005) observed movement of oil drops in a fungal hypha and decided that they floated with the cytosol without the involvement of any molecular motors. Blebby evaginations of animal cells lack microtubules or bundles of actin filaments, which might have been involved in transport, and Torgerson and McNiven (1998) were sure that organelles got into the blebs of pancreatic acinar cells with the cytosol flow. Actin monomeres, which make up filaments of the cortex, are likely to be transported to the membrane of blebs in the same way (initially there are almost no filaments there). At any rate, it is difficult to imagine that molecular motors carry so small particles individually. However, it is also possible that they move by diffusion. Mitchison and Kirschner (1988) had diffusion in mind when they wrote about the movement of actin monomeres to the apex of the neurite growth cone. Zicha et al. (2003), however, calculated that the supply rate of globular actin (to the apex of fibroblasts) could not be achieved by simple diffusion and that active transport was necessary. At the same time, they rejected the possibility of involvement of transport motors into the delivery of G-actin and thought that it was carried with the cytoplasm flow directed forward by contraction of the cell body. There are other instances of intracellular transport in which molecular motors are unlikely to be involved.

Spermatozoa of nematodes (Ascaris, Caenorhabditis) lack both actin filaments and microtubules (Nelson at al., 1982) but materials move in the cell nevertheless, in particular forwards to the newly formed areas of the apical plasmalemma. By general consent, secretory (exocytotic) vesicles carrying building material to the growing apex of walled cells are transported by molecular motors (Fischer-Parton et al., 2000; Vidali and Hepler, 2001; Taheri-Talesh et al., 2008; Cai and Cresti, 2009). At the same time, all these authors are unanimous in the opinion that longitudinal actin filaments do not enter the subapical region; for instance, filament-free zone in the pollen tube of Lilium may reach $20 \mu \mathrm{m}$ (Miller et al., 1996). This caused some bewilderment so Zonia and Munnik (2008) paid special attention to the problem. They found that in the tobacco pollen tube exocytosis occurred at some distance from the apex. On the contrary, Taheri-Talesh et al. (2008) emphasised that in the hypha of Aspergillus exocytosis occurred at the very leading edge. Actually, the data of Zonia and Munnik (2008) also indicate that endocytic vesicles move into the tube from the very apex, i.e. they somehow cross the zone free from axial actin filaments.

As we can see, directed cytoplasmic flows are not so rare. They might well be involved in intracellular transport in various motile cells. It is true that these flows have not been observed in animal cells but then the transport of substances by molecular motors has never been observed directly, either. At the same time, we should bear in mind that most of the substance transported to the 
apex is liquid (cytosol). Molecular motors certainly do not transport that. It is assumed that the cytoplasmic flow is generated by cortical contraction (see above, $\mathrm{Zi}$ cha et al., 2003) but the contractility of the cortex looks highly dubious in the light of the above considerations. It seems much more feasible that the flow is generated by influx and efflux of water (see Fig. 7).

If animal cells move in the same way as the amoeba does, why do we not see a forward cytoplasmic flow in them? The reason may be simple: it is difficult to notice. The intensity of the flow transporting building material to the apex should be directly proportional to the intensity of movement. It is much lower in animal cells than in A.proteus, which moves, relatively speaking, at breakneck speed. In slow-moving amoebae of other species the endoplasmic flow is very weak, which I have often observed on the videos.

An advantage of the running water model is that it embraces the cell as a whole and unites together the movement of its parts. Another advantage is that it does not endow moving cells with any exceptional properties. What do we see in Fig. 7? A familiar pulsation. Cell division, as we have seen before, can also be reduced to the pulsatile activity. In both cases some complexity is added to plain pulsation (Fig. 6, top row): during division the complexity is associated with the presence of the limiting equatorial ring (Fig. 6, bottom row) and during locomotion, with the alternating weakening of the sheath at the leading edge and at the tail end (Fig. 7). Two seemingly different processes, division and locomotion, turn out to be modifications of the same universal action: pulsation.

The "running water" model was developed to explain the mechanism of cell locomotion but it can also be applied to other aspects of cell life. If it is correct, we will have to admit that by localised influx and efflux of water cells can generate "specialised" cytosol flows and with their help "purposefully" transport various substances across the cell. Water exchange with the environment is a universal characteristic of cells, and the formation of cytoplasmic flows on its basis may also turn out to be a common phenomenon. Cells might localise water influx and efflux by incorporating aquaporins into certain areas of their membrane. Non-uniform distribution of aquaporins (in the plasmalemma of neutrophils) was indeed reported (Loitto et al., 2002); moreover, their concentration was especially high in the evaginating membrane.

\section{Muscle contraction}

Muscle contraction theory has been developed using evidence mostly from striated muscle fibres, which have a very strict internal organisation. Their contractile unit is the sarcomere (see Fig. 8). It is represented by a threedimensional lattice composed by thick myosin filaments and thin actin filaments arranged in parallel to each other. Actin filaments are attached to Z-disks separating the neighbouring sarcomeres.

The contractile process has long been thought to be associated with actin and myosin, the major muscle proteins. Initially it had been assumed that myofilaments shorten. Later it transpired that they do not change their length during muscular work but move relatively to each other. The sliding filament model was born on this basis. According to it, contraction results from interaction of actin and myosin filaments. Heads of myosin molecules were assumed to be the motors converting chemical energy into mechanical energy. These heads stretch to the actin filaments as cross-bridges, attaching to them and detaching from them periodically in the course of contraction. Having attached to the filaments, cross-bridges turn and pull actin filaments towards the middle of the sarcomere. Z-disks follow the filaments, and the sarcomere shortens. Cross-bridges work by conformation changes of myosin heads associated with ADP phosphorylation and ATP dephosphorylation (Rayment et al., 1993a,b).

The sliding filament model, which we will also refer to as the cross-bridges model or the model of molecular motors, quickly occupied the dominant position in the realm of ideas about muscle contraction. "In 1972 the field of actomyosin interactions was summarized in a conference at Cold Spring Harbor... After this meeting many participants thought that the problem of muscle contraction was solved 'in principle" (Cooke, 2004, p. 643). However, the final solution has never been delivered. Our imperfect understanding of the nature of muscle contraction is a source of unceasing complaints: "Over a century has passed since Kühne first extracted the proteins actin and myosin from muscle..., yet the molecular origin of the force produced between these two components remains one of the outstanding puzzles in biology" (Block, 1996, p. 151); "And perhaps it is only when molecular and integrative perspectives are taken together that we can suddenly appreciate just how far we are from understanding the contractile event" (Gunst and Fredberg, 2003, p. 415); "...although there is a general consensus that tension generation is produced by a swing of the lever arm in myosin heads attached to actin, many uncertainties remain" (Offer, 2006, p. 205). It is especially enlightening to read how the founders of the sliding filament model summarised its state after half a century of research. While H.E. Huxley (2004, p. 1413) was generally optimistic: “...I really do believe that, altogether, there is now incontrovertible evidence for the correctness of the tilting lever-arm model, although of course many important details still remain to be worked out", A. F. Huxley (2000, p. 1194) emphasised the challenges: "The preceding paragraph mentions three recent observations that have not yet been incorporated into current theory, but there are many other 
unanswered questions that have been with us for many years." He also added: "Apart from these recognized uncertainties, there is always the possibility that something totally unexpected [emphasis mine - VB] will turn up, as happened with the disproof of the lactic acid theory and the discovery of sliding filaments."

However that may be, most researchers studying muscle contraction take it for granted that it is myosin heads that perform the mechanical acts (see, e.g., Harrington, 1979; Baños et al., 1996; Houdusse, Sweeney, 2001; Tesi et al., 2002; Masuda, 2003; Lampinen, Noponen, 2005). Discussions mostly focus on the way in which the heads work. One should bear in mind, however, that this hypothesis is not supported by any direct evidence. No one actually observed the movement of cross-bridges. It is just known that they may be tilted at various angles to actin filaments and, on the whole, the available evidence is contradictory (see Martin-Fernandez et al., 1994). The authors themselves (Martin-Fernandez et al., 1994) found that cross-bridges did not always change their orientation during muscle contraction. They hypothesised that "...in isometric contractions the change in axial orientation (of cross-bridges - VB) is not the cause of active tension production, but rather the result of it" and admitted the possibility of "....an alternative hypothesis for contraction..." (p. 319).

In case of smooth muscles, the sliding filament model encounters many more difficulties. Structurally, smooth muscle cells are similar to striated fibres only in the fact that they contain abundant actin and myosin filaments arranged in parallel to the longer cell axis. However, they do not demonstrate regular organisation (Tyreman and Molloy, 2003); “...the sarcomeric structure akin to that in striated muscle, which allows the sliding of contractile filaments to be translated into cell shortening has yet to be elucidated" (Herrera et al., 2005, p.2381). No sliding of myofilaments relatively to each other in the smooth muscle cells was observed. Neither did anyone observe any regular longitudinal displacement of the so-called dense bodies, putative analogues of Z-disks in sarcomeres. There are different opinions about the relative length of myosin and actin filaments, their arrangement and the way in which myosin heads work (see Kargacin et al., 1989; Small et al., 1990; Xu et al., 1996; Herrera et al., 2005). Generally, when discussing the organisation of the contractile apparatus in smooth muscles, the researchers usually admit that "The intracellular organization of contractile filaments... is still poorly understood" (Kuo and Seow, 2004, p. 1503). I would like to add that, contrary to the myofilaments of striated muscles, the actin filaments (Gunst and Fredberg, 2003) and possibly also the myosin filaments (Seow et al., 2000) of smooth muscles are unstable, being partly depolymerised during cell relaxation and polymerised (restored) during its activation.
Some structural and functional differences between smooth and striated muscles are especially intriguing. A smooth muscle cell contains, relatively, much less myosin and therefore much fewer molecular motors than a striated fibre; the difference may be quintuple (Ivanov, 1950; Murphy et al., 1974; Dillon and Murphy, 1982; Warshaw et al., 1987). Despite that, a smooth muscle may generate the same or even a greater force per cross-section unit than the striated one (Murphy et al., 1974; Dillon and Murphy, 1982). Some in vitro observations show that the myosin head of a smooth muscle is much more efficient than that of a skeletal muscle (VanBuren et al., 1994). This phenomenon is more often explained by longer periods of cross-bridge attachment to actin filaments in smooth muscle cells (Siegman et al., 1980; Dillon and Murphy, 1982; Murphy, 1994; Guilford et al., 1997; Butler et al., 2001).

Another difference between smooth and striated muscles is their ability to shorten. Striated muscles of vertebrates normally shorten by $25-60 \%$ and those of insects shorten even less (Edwards et al., 1956). In contrast, smooth muscle cells of vertebrates may shorten four times (calculations based on my measurements of cells in Fay, Delise, 1973, Fig. 1) or even five times (Draeger et al., 1990). The structural basis of the ability to shorten so much is poorly understood (Xu et al., 1996).

The most fascinating thing about smooth muscles is that they expend very little energy for their work. The expenditure of chemical energy by a tonically contracted smooth muscle is tens or even hundreds of times (300400) smaller than that of a tetanically contracted skeletal muscle generating the same force; energy consumption does not correlate directly with the tension and a tense muscle shows no apparent fatigue (Ivanov, 1950; Glück and Paul, 1977; Siegman et al., 1980; Somlyo and Somlyo, 1994). For instance, the internal anal sphincter in humans is almost always in the state of maximum contraction.

In the light of the above, saying that "Smooth muscle exhibits biophysical characteristics and physiological behaviors that are not readily explained by present paradigms of cytoskeletal and cross-bridge mechanics" (Gunst and Fredberg, 2003, p.413) is putting it mildly. Moreover, a certain feature characteristic of both smooth and striated muscles puts all muscles into antagonism with the cross-bridges theory.

If muscular force is generated by molecular motors, it should be directly proportional to number of these motors and thus to the size of the muscle, its thickness and length. In reality, the force produced by a muscle is not correlated with its length, i.e. it does not depend on the number of molecular motors. The force is correlated only with the muscle thickness. More precisely, it is directly proportional to the area of the physiological section of the muscle, which is perpendicular to 
the longer axis of muscle fibres or smooth muscle cells. Muscle force is calculated per unit area of this section, e.g. $\mathrm{kgf} / \mathrm{cm}^{2}$.

These facts do not just contradict the theory of molecular motors. They effectively disprove it, jettisoning the entire modern theory of muscle contraction. At the same time, they point to a new way to the solution of the problem. "Biological forces from muscle are proportional to the cross-section of the muscle, and scale as $\left[\mathrm{s}^{2}\right]$. Pneumatic and hydraulic forces are caused by pressures (P) and also scale as $\left[\mathrm{s}^{2}\right]$ " (Trimmer, 1989, p. 274). This way, as we see, leads us into the field covered by hydrostatic forces. Several features in the behaviour of smooth muscle cells indicate that they might employ hydrostatic mechanism in their work.

Contracting isotonically under experimental conditions, smooth muscle cells decrease in volume (ca. by $20 \%$ in the experiments of Fay and Delise, 1973). Clearly, this can only happen by water loss. Kargacin and Fay (1987) thought that water was removed from the cell mechanically during cell contraction. In an earlier discussion of various invaginations I have already expressed my doubts about the mechanical removal of water. Smooth muscle cells offer a good possibility to test this hypothesis using evidence from the following experiment. During isometric contraction (i.e. when the length remains unchanged) of smooth muscle cells their cross-section decreased (Gillis et al., 1988), which meant that the volume and the water content decreased too. The contraction of the actomyosin complex could not be the reason since myofilaments of smooth muscle cells stretched along the longer axis, not crosswise, as specially noted by the authors. The only possibility remains: at least in this case the cell decreased their volume by active removal of water. The idea that smooth muscle cells can actively remove water and even make their internal pressure lower than the pressure outside is indirectly supported by their ability to produce numerous blebby invaginations called surface vesicles (Devine et al., 1972) or caveolae (Bond et al., 1984).

Relaxing after contraction, smooth muscle cells restore their initial length and volume (Fay and Delise, 1973; Ives et al., 1978). One of the explanations of this phenomenon was that some structures inside the cell were compressed during contraction and when an active contractile event stopped, the accumulated forces moved the cell ends apart (Warshaw et al., 1987). The authors did not explain, however, how such a "spring" might work. Theoretically, the cell ends may be pushed apart by actin filaments. This explanation is given for non-muscle cells (see above) but in this case it seems even less plausible if only because it is during relaxation that actin filaments disassemble (see Gunst and Fredberg, 2003). The main thing, however, is that cells, relaxing and elongating, restore their volume, and this may only happen by the uptake of water lost during contraction. Kargacin and Fay (1987) decided that the enlargement of smooth muscle cells during relaxation was due to osmotic water uptake. Indeed, this seems to be the only possible explanation.

So, we can be fairly sure that smooth muscle cells actively remove water and take it up again, decreasing or increasing their cytoplasmic volume and internal pressure in this way, and that they use these operations for shortening (contraction) or elongation of their body. Basically, smooth muscle cells perform actions which are called RVD and RVI in case of non-muscle cells. Similarly to other cells, muscle cells pulsate. The only difference is that their pulsation occurs at a much larger scale, and we call its phases contraction and relaxation.

The application of the hydrostatic mechanism of contraction to smooth muscle cells makes it easy to explain their functional features mentioned above, first of all, a low energy expenditure of the contractile process and the indefatigability of muscles. A contracting cell only expends energy for the removal of water (the establishment of the osmotic gradient), while the mechanical work is done by the pressure of the outer mass. Having lost some water and contracted, a muscle cell transits into a new state and is maintained in it by the same outside pressure. Essentially, the contracted state does not differ from the relaxed state, and its maintenance requires the same amount of energy. A contracted smooth muscle cell may be likened to the famous Magdeburg hemispheres, which were firmly held together by the atmospheric (external) pressure.

If smooth muscle cells shorten by removal of water, it is unsurprising that they can shorten considerably. One other fact, however, is surprising indeed. Contracting isotonically, cells do not only shorten but also thicken in their middle part; elongating during relaxation, they become thinner again (Fay and Delise, 1973; Fisher and Bagby, 1977; Warshaw et al., 1987; Kargacin et al., 1989). A cavitary body cannot decrease its internal pressure, increasing at the same time its linear size; by the same token, its size cannot decrease if the pressure increases. We have come across this problem once already when discussing various processes in non-muscle cells. Then I made an assumption that a change in the internal pressure (the volume of the inner mass) was an oscillating process, with the periods of decrease alternating with periods of increase. Let us now extend this solution to smooth muscle cells. One might assume that their volume decreases not all the time during contraction (shortening); it increases periodically, and the cell thickens in these moments. Similarly, an increase in the cell volume during its relaxation (elongation) at times alternates with its decrease, and then the cell becomes thinner (and produces endocytic vesicles on the way see above). A regular alternation of contraction and re- 
laxation has indeed been found in various smooth muscles in vivo as well as in vitro (Kasai et al., 1994; Haddock and Hill, 2005). Small oscillations of tension in muscle cells superimposed on the tonic contracture were also found (Bond et al., 1984).

Importantly, the hydrostatic model of smooth muscle cells' functioning may be supported by analogies with familiar motors, that is, industrial engines. In a steam engine or an internal combustion engine, the working stroke of piston is produced due to the difference of the internal pressure (in a closed chamber) and the external pressure. The first steam engines constructed in the late XVII $I^{\text {th }}$ - early XVIII ${ }^{\text {th }}$ century are an especially close analogy. Not only did they operate on the basis of the pressure difference but they operated exactly in the way assumed by the new hypothesis. The piston of these engines performed the working stroke under the impact of external (atmospheric) pressure when the condensation of cooled steam created a partial vacuum in the cylinder. These engines are called, fittingly enough, atmospheric steam engines.

It is especially significant that the hydrostatic mechanism is also used by living machines - plant cells, driving the movement of parts of the entire organism. In the evolution of plants "...the ability to reversible motions based on changing turgor pressure develops increasingly more often [emphasis mine - VB]. This mechanism evolved when stomata were formed in the earliest land higher plants. Then plants began to use slow turgor (nastic) movements for the moving leaves, opening and closing flowers. Finally, they evolved seismonasties - fast reversible turgor movements" (Polevoy, Salamatova, 1985, p. 191). Noteworthy, the terms "flexors" and "extensors", which are usually applied to muscles, are also used for describing the motor plant cells. Were it not for the firm belief in the abyss dividing the plant cell and the animal cell in mechanical respect, the researchers of muscle contraction might have long noticed the contraction of plant cells.

Pressure difference determines the movement of masses in many other natural and anthropogenic processes. High pressure drives bullets out of barrels, lava out of volcanoes, water and steam out of geysers; a pressure difference generates the lift force of an airborne plane or a swimming shark; winds and currents arise to a large extent due to the pressure difference in the neighbouring parts of the atmosphere or the ocean. Alongside with gravity, pressure difference is a key primary factor causing rectilinear movements of masses in inorganic nature and chemically-fuelled industrial engines. All the transformations of living cavitary bodies discussed above fit this scheme. The contraction of smooth muscle cells fits it, too.

Striated muscles are similar to smooth muscles in that the force they produce is directly proportional to their cross-section (physiological section). One might expect them to generate this force in the same way, too. Yet muscles are quite different in the structure of their contractile units. A smooth muscle cell is a closed cavitary body, while the sarcomere is an open three-dimensional lattice. For a hydrostatic mechanism to act there should be a pressure difference between the cavity of a living body and the environment. Can the pressure inside an open sarcomere be different from the pressure in the surrounding sarcoplasm? In principle, it can. Pressure difference in open environments is common, e.g., between neighbouring areas of the atmosphere. The feature of closed living cavitary bodies is not that their internal pressure may be different from the external one but that they actively generate and regulate this difference. Cells, including muscle cells, do so by transporting water across the semipermeable membrane along the osmotic gradient. When a muscle cell contracts, water leaves it. When sarcomeres contract, water leaves them, too (Carlsen et al., 1961), to return later. How does this happen?

Water exchange between sarcomeres and the environment has been observed in the experiment more than once. Partially or completely demembranated muscle fibres placed into relaxation solution immediately after the destruction of the sarcolemma took up water and swelled (Matsubara and Elliott, 1972; Gordon et al., 1973). The distance between myofilaments increased in the process, and the sarcomeres thickened (Godt and Maughan, 1977; Goldman and Simmons, 1986). After the fibres were transferred into a hypertonic solution, they contracted and returned to their initial appearance. An osmotic gradient is also periodically established during contraction of natural fibres. The process of their contraction and relaxation is correlated with the fluctuation of the concentration of the sarcoplasmic $\mathrm{Ca}^{2+}$. When calcium is released from the cisterns of the sarcoplasmic reticulum into the cytoplasm around myofibrils, it increases its tonicity. This establishes an osmotic gradient between myofibrils and the surrounding sarcoplasm. Water might leave the sarcomeres along this gradient. It might, were it not for a certain circumstance.

Just now, describing the experiments with demembranated muscle fibres, I kept quiet about what the hypertonicity of the cultural solution was due to. It was due to large molecules (e.g., dextran) incapable of passing between myofilaments and entering the sarcomere. Calcium ions, on the other hand, are quite different from the large molecules of dextran. They may easily diffuse into the sarcomere lattice eliminating the osmotic gradient. It has been calculated that the concentration of calcium ions inside and outside the fine myofibril (1-3 $\mu \mathrm{m}$ in diameter) is equalised in less than a millisecond (Telley and Denoth, 2007). Would there be time for water to leave sarcomeres before calcium ions get there? 


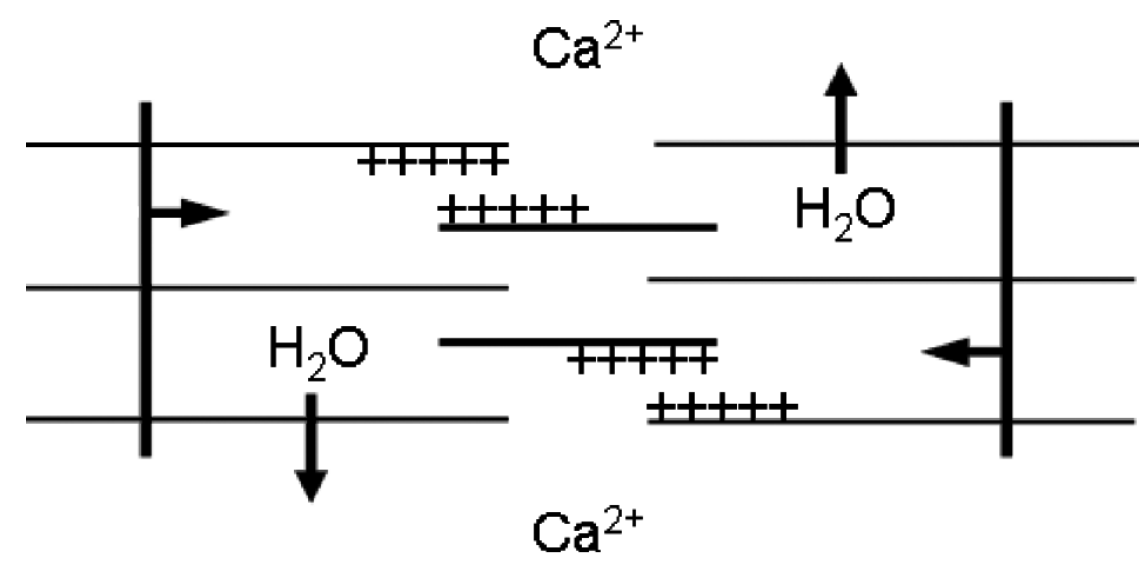

Fig. 8. Mechanism of sarcomere shortening in a striated fibre (a scheme).

Thin horizontal lines - actin filaments; thick horizontal lines - myosin filaments; vertical lines - Z-disks. Calcium ions $\left(\mathrm{Ca}^{2+}\right)$ leave the sarcoplasmic reticulum and enter the sarcoplasm, increasing its tonicity. The net electric charge of the filament lattice becomes positive, this prevents the penetration of calcium ions into the sarcomere. Water $\left(\mathrm{H}_{2} \mathrm{O}\right)$ leaves the sarcomere along the osmotic gradient (vertical arrows), a lowered pressure is created in the sarcomere. Similar (positive) electric charges on myosin and actin filaments (+++++) prevent them from coming together. Z-disks move (horizontal arrows) into the zone of lower pressure, the sarcomere shortens.

Theoretically, there is a way to prevent calcium ions from entering the sarcomeres. The net positive charge of the filament lattice might be such a barrier. In general, this charge is variable. Its sign depends, for instance, on the $\mathrm{pH}$. At $\mathrm{pH}=5$, the charge is neutral; when $\mathrm{pH}$ increases above this level, it becomes negative; when it decreases below five, it becomes positive (Elliott, 1973; Godt, 1981; Naylor et al., 1985; Millman, 1998; Regini and Elliott, 2001). Electrostatic characteristics of myosin and actin filaments might depend on the availability of free ATP. At any rate, myofilaments of muscle fibres placed into relaxation solution (with ATP present) and into rigor solution (with ATP absent) had different charges (Bartels and Elliott, 1985).

In the light of the above, a possible "hydromechanical" scenario of the contraction of the sarcomere of a striated muscle fibre might be represented as follows (Fig. 8). Calcium ions are released from the sarcoplasmic reticulum into the sarcoplasm, increasing its tonicity - the net electric charge of the sarcomere becomes positive (possibly, as a result of nucleotide transformations) - because of this, $\mathrm{Ca}^{2+}$ does not diffuse into the sarcomeres and the water leaves them by osmotic gradient - a lower pressure is created in the sarcomere - similar (positive) electric charges on myosin and actin filaments prevent them from coming together Z-disks move into the zone of lower pressure, and the sarcomere shortens.

Smooth muscle cells might also possess the ability to contract according to the "sarcomere" pattern. Experimentally demembranated smooth muscle cells retained their contractile function in the presence of calcium and
ATP (Small, 1974; Saida and Nonomura, 1978; Kargacin and Fay, 1987; Bialojan et al., 1988; Kargacin et al., 1989; Butler et al., 2001; Niiro and Ikebe, 2001; Wilson et al., 2002) though generated a lesser force than intact cells (Arner, 1983; Kossmann et al., 1987). In smooth muscle cells there are also actin and myosin filaments arranged in parallel to each other. After a demembranated cell becomes an open system, they might act similarly to the myofilaments of sarcomeres, i.e. change their electric charge on the filament lattice.

New ideas about the contraction of striated muscles do not contradict the basic facts underlying the sliding filament model. First of all, this concerns the sliding itself, which gave the model its name. In the traditional scheme, actin filaments slide because myosin heads pull them, and also pull Z-disks together with them. In the hydromechanical model, Z-disks push actin filaments, that is, the sliding of filaments is the consequence of sarcomeres shortening rather than its cause. One may wonder why actin filaments pushed by Z-disks are not deformed. Their deformation may be prevented by electrostatic forces helping to maintain some distance between the myofilaments (see Elliott et al., 1970; Millman and Nickel, 1980; Irving et al., 2000). It would be pertinent to recall the words of Huxley (1969, p. 1360), who wrote that myofilaments slide "...on a cushion of long-range electrostatic forces..."

Another fact, which occupies an important place in the modern theory of muscle contraction, is a positive correlation between the degree of overlapping of actin and myosin filaments (up to a certain limit) and the force generated by the sarcomere. This correlation 

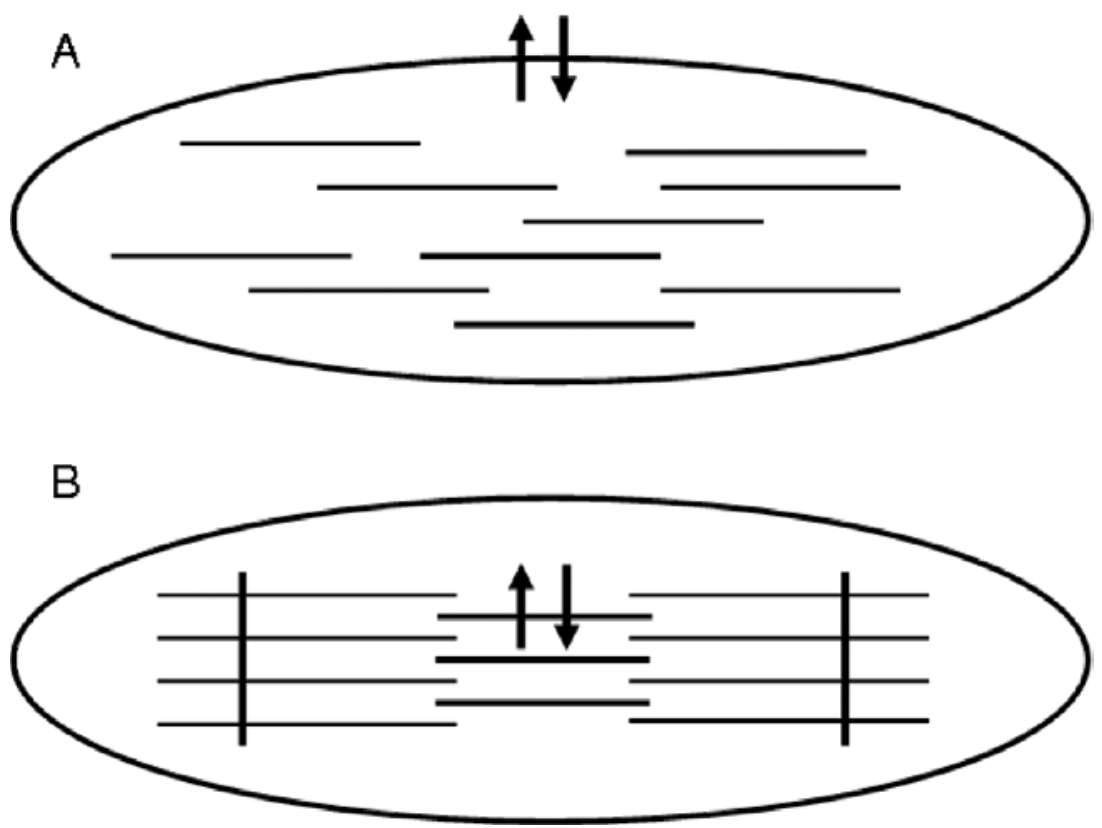

Fig. 9. Movement of water along the osmotic gradient during contraction and relaxation of a smooth muscle cell (A) and a striated fibre (B) (a scheme).

The cell and the fibre are shown as ovals. Thin horizontal lines - actin filaments; thick horizontal lines - myosin filaments; vertical lines - Z-disks. A: During contraction water leaves the cell (upward arrow), during relaxation water enters the cells (downward arrow). B: During contraction water leaves the sarcomere (upward arrow), during relaxation water enters it (downward arrow). Structural changes of the muscle cell and muscle fibre are not shown.

is thought to be associated with the fact that the number of myosin heads (motors) binding to actin filaments depends on the degree of overlapping. The role of the heads, however, is not purely mechanical. ATP hydrolysis occurs on them, its rate increasing manifold in the presence of filamentous actin (Lymn and Taylor, 1971; Geeves, 1991). The greater number of myosin heads neighbour actin filaments, the more energy is generated. Besides, mutual penetration of myofilaments increases the number of opposed similar charges, which increases the separating force and prevents crosswise contraction (thinning) of sarcomeres. Quite some time ago Elliott et al. (1970) thought that electrostatic repulsion between filaments could play a key part in the contractile process.

So, according to the new hypothesis, the contraction of sarcomeres and smooth muscle cells is literally their compression by the outer mass. This compression occurs after sarcomeres or cells lose water and their inner pressure becomes lower than the pressure outside. If we agree that the contraction is caused by the removal of water from contractile units, it would be easier to understand one more difference in the work of striated and smooth muscles, namely, the former contract much faster than the latter. The reason behind this difference may be the different speed with which the water leaves the muscle cell (Fig. 9A) and the sacromere (Fig. 9B). It is clear that water may leave an open sarcomere faster.
Besides an increased rate of contraction, another innovation of striated muscles was the ability for a finer regulation of the contractile process. This is also easier to explain within the framework of the new model. In smooth muscles water is exchanged between the cell and its environment (Fig. 9A). Chemical composition of the environment is more or less variable, and smooth muscle cells, as any other cells, respond to these changes at once. In striated muscles water is exchanged inside the closed cavity of the fibre, between sarcomeres and the sarcoplasm (Fig. 9B), where the stability of the environment is maintained more rigorously. The tendency towards increasing the stability of the life milieu is in general characteristic of animal evolution. Examples of this tendency are transitions from embryonic-larval to an entirely embryonic development or from poikilothermy to homothermy. It cannot even be ruled out that in case of muscles it was the transition of the contractile apparatus to functioning in a relatively invariable environment (inside the fibre - Fig. 9B) that "helped" the locomotor (skeletal) musculature to become voluntary.

Extending the hydromechanical model to muscle contraction, we can explain both the similarity (force being proportional to cross-section) and the differences (energy expenditure, speed of contraction etc.) in the work of smooth and striated muscles. I do not know any other hypothesis that accounts for all these things 
together. At the same time, the new model gives rise to new questions. If the muscle is "contracted" by the outer mass, in land animals the force of contraction should be ultimately determined by the atmospheric pressure. This means that the force should not exceed one kilogramme per one square centimetre of section. However, the literature data indicate that this force may be much greater than that.

Muscle force has been measured in intact muscles, muscle cells, muscle fibres, and myofibrils. The force has been expressed in various units (newtons, dynes etc.), and square microns, square millimetres etc. were taken as a section area unit. I recalculated all these data for $\mathrm{kgf} / \mathrm{cm}^{2}$. Data on the same object are grouped together and values in the group are arranged in the ascending order. Myofibril of skeletal muscle: 1.59 (Friedman and Goldman, 1996); 2.61 (Yuri et al., 1998); 3.7 (Colomo et al., 1997); 3.43-9.58 (Bartoo et al., 1993). Myofibril of the cardiac muscle: 3.5 (Colomo et al., 1997). A smooth muscle: 0.13-2.2 (reviews by Herlihy, Murphy, 1973, 1974, after Murphy et al., 1974); 0.34 (Arner, 1983); 0.51 (Warshaw et al., 1986); 6.83 (Dillon and Murphy, 1982). Cardiac muscle: 1 (Brutsaert, Housmans, 1977, after Colomo et al., 1997). Skeletal muscle: 1.47-2.94 (Close, 1972, after Murphy et al., 1974); 2-3 (Bagshaw, 1993, after Colomo et al., 1997); 3.57 (Close, 1972, after Dillon and Murphy, 1982).

We can see that the scattering of data is considerable. Extreme values should probably be discarded but even the values of $2-3 \mathrm{kgf} / \mathrm{cm}^{2}$ (from the review by Bagshaw) are much larger than the atmospheric pressure. Nevertheless, there is a way out from this impasse.

Smooth muscle cells thicken in their middle part during contraction. My measurements of cells shown at micrographs indicated that their radius increased ca. by a factor of 1.3 (Kargacin and Fay, 1987, Fig. 8), 1.5 (Fay and Delise, 1973, Fig. 2) and 1.7 (Fay and Delise, 1973, Fig. 1). Separately tested myofibrils of striated muscles also thicken during contraction, and do so along their entire length. According to my measurements, their radius increased ca. by a factor of 1.3 (Hanson J. 1952, Fig. 1) and 1.7 (Lionne et al., 2003, Fig. 1). In these cases the maximum increase of the cross-section area made up almost three times in both smooth muscle cells and myofibrils. Since the force is directly proportional to the section area of the muscle cell or the muscle fibre, it follows that the force also increased threefold in the process of contraction.

The figures obtained in calculations of the muscle force may be considerably overestimated if we take into account only the initial section of a contracting body and disregard its growth in the course of contraction. For instance, we measure the section area of a relaxed muscle and get the figure of $1 \mathrm{~cm}^{2}$, then measure the force it develops during contraction and get the figure of two kilogrammes. Based on these measurements, we obtain a relationship of $2 \mathrm{kgf} / \mathrm{cm}^{2}$. In reality, this force might have been produced not by the "initial" muscle but by a muscle with a doubled section area. Incidentally, muscles generate the maximum force gradually rather than at once.

So, muscles work similarly to atmospheric steam engines. This sounds "totally unexpected" (see the above quotation from Huxley, 2000) but it is the only hypothesis so far that explains the fact that the force produced by muscles is proportional only to their cross-section area (physiological section).

At present it is believed that the ability to contract is characteristic of actomyosin complexes not only of muscle cells but also of non-muscle ones. As concerns the former, this opinion is based on a totality of observations and arguments arranged in the sliding filament hypothesis. The ideas about actomyosin contraction in non-muscle cells are not based on a similarly extensive body of evidence, being to a large extent developed by analogy with muscle contraction. The rejection of the model of molecular motors entails the rejection of the idea of actin-myosin contraction in all its variants. Its last resort are experimental data (Toyoshima et al., 1987; Umemoto, Sellers, 1990; VanBuren et al., 1994; Guilford et al., 1997) showing that myosin and actin may move against each other and generate force in the process. Unfortunately, it is difficult to say to what extent this interaction of myosin and actin in vitro conforms to their interaction in vivo. According to the sliding filament model, each myosin head of intact muscles performs the working stroke (pulls the actin filament) only in one direction, while in the experiments it moves also in the reverse direction (Guilford et al., 1997; Yanagida et al., 2000). Based on this, it was suggested (Yanagida et al., 2000) that the movement of myosin in vitro is merely Brownian motion.

The arguments presented in this section and elsewhere in the paper indicate quite clearly that there is no "contractile" interaction between actin and myosin filaments in nature. At the same time, an old belief that the force-generating mechanism in non-muscle cells and muscle cells (fibres) is the same turned out to be prophetic. This universal mechanism, however, is now seen in a different light.

\section{General conclusion}

\section{A UNIVERSAL MODEL}

We have discussed mechanical processes transforming the shape of cells, protists, whole embryos and larvae and their organs. We have considered various models describing these processes to find out that many old questions are still to be answered. Even the hypotheses 
generally accepted by the scientific community such as the model of cell division or the model of muscle contraction face a number of almost insurmountable difficulties.

The answer suggested in this paper is quite unlike the others. It is a general scheme explaining entirely different processes rather than the models describing a specific phenomena. I came to this solution after becoming deeply aware of the fact that all living organisms and many of their parts are organised in an essentially similar manner: they are closed cavitary bodies.

A cavitary body consists of two parts: 1) the inner mass and 2) the sheath serving as a mechanical barrier between the inner mass and the environment (the outer mass). Hypotheses dealing with morphogenetic processes in cavitary bodies usually consider the action of either the inner mass only (for instance, during the development of limb buds) or the sheath only (for instance, during the development of endodermal and ectodermal rudiments). The outer mass is outright ignored. According to the new model, a change in the size and shape of cavitary bodies always results from the interaction of three components: 1) the inner mass, 2) its sheath and 3) the outer mass.

Whatever the composition of the inner and the outer mass, the sheath is usually in contact either with a liquid or with a semifluid extracellular matrix; both exercise a uniform pressure on the sheath (Fig. 2A). Actively taking up and taking out substances, first of all, water, a cavitary body changes the volume of the inner mass, in this way creating and regulating the difference between the internal and the external hydrostatic pressure (Fig. 2B, C). The inner (the outer) mass tends to move into the zone of the lesser pressure but the sheath is a barrier on its way. As the pressure difference grows, the inner (the outer) mass overcomes the resistance of the sheath, displaces it outwards (inwards) and moves together with it. Displacements occur earliest or at all at the sites where the sheath offers the least resistance to the pressure. These local displacements are evaginations (Fig. 2B) and invaginations (Fig. 2C). It is these displacements that change the shape of the body or its parts. The degree of the sheath's resistance to the pressure of the adjoining masses is determined by its own properties (microstructure, thickness, curvature etc.) and, in case of some evaginations, also by the presence or absence of linkers between contralateral areas of the sheath (Fig. 5). When pressure inside cavitary bodies, at least unicellular ones, changes actively, the phases of its increase and decrease usually alternate. Specific actions of cells such as division, locomotion or contraction are a modification of their routine pulsatile activity affected by means of an alternating influx and efflux of water (see Figs. 6; 7;9A).

In one form or another, ideas about an important morphogenetic role of the hydrostatic pressure have been suggested more than once before. Turgor pressure and mechanical heterogeneity of the sheath have long been accepted as a condition of the non-uniform growth of walled cells. Changes in the cell turgor are thought to cause movements in plants. Increased pressure inside animal cells is thought to underlie the emergence of blebs. The difference between the external and the internal pressure has been regarded as the cause of invaginational gastrulation. The model assuming that the difference in the internal and the external hydrostatic pressure is the major morphogenetic factor has now been extended (as hydromechanical model - Fig. 2) to many more objects and processes. It can be considered as a truly universal model.

\section{MECHANICAL FACTORS IN \\ THE EARLY ONTOGENESIS OF VERTEBRATES}

In order to highlight the importance of "hydromechanical" factors, let us consider, in a purely narrative manner, how they might act in ontogenesis. Urodele amphibians will serve as protagonists in this scenario.

The major trend of the embryonic and larval development is an increase in size (growth) of the entire organism and its parts when the internal pressure is higher than the external one; various evaginations are formed during that time. There are periods, however, when pressure in some cavities falls, and then invaginations arise. In more detail, the development proceeds as follows.

At the early stage of zygote cleavage, the cavity of the nascent blastula communicates freely with the outside liquid and its internal pressure is the same as the external one. The sheath of a mature blastula is less permeable, and the embryo now can actively regulate the internal pressure. Osmotic concentration in the cavitary fluid decreases as compared to that in the environment, water starts to leave the blastocoel and the pressure in it becomes lower than the pressure outside. The outer liquid bends the blastoderm and presses (invaginates) it into the blastula cavity: gastrulation occurs (see Fig. 2C). After the closure of the blastopore, a closed cavitary body, the gut, is formed in the primary cavity of the embryo. The space between the epithelial sheath of the entire embryo (the ectoderm) and the epithelial sheath of the gut (the endoderm) is occupied by free cells (the mesoderm).

Following gastrulation, the organism mostly grows but at times the pressure inside the primary body cavity drops below the outside pressure again. In such periods the neural tube, auditory vesicles and olfactory sacs arise by invagination into the primary cavity; the external liquid invaginates in the area of placodes where the surface of the embryo is flattened. The cells of the dorsal mesoderm are packed into somites, while its ventral cells form the walls of the coelomic cavity. Outgrowths 
of mesodermal cells and collagen fibres (linkers) are attached to the ectoderm and the internal organs connecting them with each other (see Fig. 5A). The growing inner mass pushes the ectoderm off the centre; the ectoderm, connected with internal organs by linkers, in its turn pulls somites and parietal walls of the coelom in the same direction (centrifugally). Somites attached to the central organs do not bear the strain and their inner and outer epithelial walls are destroyed. Coelomic walls maintain their integrity, with the traction of the centrifugally moving ectoderm "ripping" only some of the cells out of them (see Fig. 3E, F). Finally, the space between the cavitary embryonic organs is again occupied by free cells now referred to as mesenchymal cells (see Fig. 5B). The mesenchyme gives rise, in particular, to the skeleton, whose cartilaginous and bony rudiments are formed from dense accumulations of cells. We will leave them as they are. Our further discussion will concern only the transformations of the cavitary organs.

After the disintegration of somites, collagen linkers are mostly retained in the region of transverse myosepta, where they limit the centrifugal displacement of the ectoderm as they did before. At these sites furrows appear on the body and its surface becomes wavy (Fig. 4D; see also Fig. 5B). Hindering the centrifugal displacement of the ectoderm, linkers limit the expansion of the inner mass, and the skin (first of all, the basement membrane) does the same. The growing inner mass overcomes the resistance of the ectoderm, moving it off the centre. The basement membrane is destroyed at some sites, the resistance of the ectoderm at such sites decreases and the inner mass expands especially fast: it evaginates. In this way, the tail, the unpaired fin fold (see Fig. 3C), external gills, limb buds (Fig. 4A; see also Fig. 3B), the operculum (Fig. 4B) and balancers (Fig. 4C) arise and grow. Numerous cross-linkers in the unpaired fin fold (Fig. 4E) make it flat (see also Fig. 3C).

Embryonic and larval organs such as the coelom, the gut (before the opening of the mouth and the anus), the neural tube, blood vessels and kidney tubules regulate their internal pressure autonomously. It usually exceeds the pressure in the primary body cavity, and this allows organs to grow. This growth, similarly to that of the entire embryo or larva, may be non-uniform. At the sites where the basement membrane of epithelial sheath is destroyed, the liquid filling the organs buckles the sheath and evaginates together with it into the body cavity. In this way gut derivatives (liver, lungs) arise, pronephric ducts extending from the coelomic cavity elongate and capillaries and kidney tubules bud.

The ectoderm, restraining the expansion of the inner mass, in its turn, experiences its stretching action, and for this reason the skin epithelium remains uniserial for a long time. Only at late larval stages, when the growth of the inner mass slows down in comparison with the increase in the area of the skin, the stretching effect weakens and the epidermis becomes multiserial. During metamorphosis the volume of the inner mass noticeably decreases and the internal pressure drops below the level of the external one. This results in local invaginations of epidermal cell layer at the sites where the basement membrane is destroyed and in the formation of skin glands there.

\section{MECHANICAL FACTORS IN THE EVOLUTION OF LIVING ORGANISMS}

The evolution of morphogenetic abilities of living organisms may be hypothetically represented as follows.

Ancient prokaryotic organisms (cells) were closed cavitary bodies. Their cytoplasm was enclosed in a single-layer sheath, the plasmalemma. They had no cytoskeleton but had specialised water channels, aquaporins, which ensured intensive water exchange with the environment. By means of alternating influx and efflux of water, prokaryotes rhythmically increased and decreased the volume of the cytoplasm - they pulsated. Decreasing the internal pressure relatively to the external one in the course of pulsation and locally weakening the plasmalemma at the same time, prokaryotes performed endocytic actions; when the internal pressure increased, exocytosis occurred. A particular modification of pulsatile activity was represented by cytokinesis. Incorporating aquaporins into certain areas of the plasmalemma, the cells created directed cytosol flows and used them for transporting various substances.

In eukaryotic unicellular organisms, a second, internal sheath layer, the cortex, was added to the plasmalemma. It consisted mostly of actin filaments. Destroying and reconstructing some parts of the cortex, they could regulate the mechanical properties of the sheath more effectively. Owing to this, the cells could increase the complexity of their shape, creating, in particular, permanent and temporary outgrowths. Having created a new modification of pulsatile activity, some cells could actively crawl by means of alternating evagination (in front) and invagination (in the rear). Amoeboid protists were especially skilled in this kind of movement.

Animals reproduced the ancient mechanical toolkit on a new constructive basis. Its formation started when some protists began to produce such elements of extracellular matrix as glycosaminoglycans, which could bind water, and collagen. Hydrated amorphous matrix ensured a uniform pressure of the inner mass upon the sheath of the primary body cavity (the ectoderm) of embryos and larvae. Collagen fibres built the basement membrane, one of the two layers of sheath of the primary body cavity and cavitary organs, while epithelial cells formed the second layer. Besides, collagen fibres formed linkers connecting contralateral areas of the ectoderm 
and preventing them from moving apart. In embryos of vertebrates, the linkers were involved in the disintegration of epithelial walls of somites; the mesenchyme was formed in this way.

As complexity of animals increased in the course of the evolution, the basic morphogenetic processes were shifted to earlier ontogenetic stages, when, in particular, cavities became more numerous. The embryo of the most complex multicellular organisms, the vertebrates, is a hierarchical system of autonomous closed cavities filled with pliable mass: extracellular matrix (the primary body cavity), vacuolated cells (notochord) and liquid (coelom, the gut, vessels, and kidney tubules). By changing their internal pressure, destroying the basement membrane in some areas of the epithelium or changing its curvature, some cavitary bodies may form invaginations and evaginations, which give rise to various definitive organs.

In general, animal cells retained the mechanical properties of protists. Their pulsatile activity proceeded at an especially large scale in smooth muscle cells, which became specialised motor units. Striated muscles evolved a unique mechanism of controlled water exchange inside closed muscle fibres, between sarcomeres and sarcoplasm. Plant cells and some other cells added the third, outer layer to its sheath: the polysaccharide wall. This rigid inert layer limited the morphogenetic and locomotor possibilities of walled cells.

\section{References}

Abraham, V. C., Krishnamurthi, V., Taylor, D. L., and Lanni, F. 1999. The actin-based nanomachine at the leading edge of migrating cells. Biophysical Journal 77:1721-1732. doi: 10.1016/S0006-3495(99)77018-9

Ahmed, N., Ramjeesingh, M., Wong, S., Varga, A., Garami, E., and Bear, C. E. 2000. Chloride channel activity of CIC-2 is modified by the actin cytoskeleton. Biochemical Journal 352:789-794. doi: 10.1042/bj3520789

Albertson, R., Riggs, B., and Sullivan, W. 2005. Membrane traffic: a driving force in cytokinesis. Trends in Cell Biology 15:92-101. doi: 10.1016/j.tcb.2004.12.008

Aletta, J. M., and Greene, L. A. 1988. Growth cone configuration and advance: a time-lapse study using video-enhanced differential interference contrast microscopy. The Journal of Neuroscience 8:1425-1435.

Allen, R. D. 1961. Ameboid movement. pp. 135-216 in Brachet, J., and Mirsky, A. E. (eds.), The cell, vol.2. Academic Press, New York, London.

Anderson, K. I., Wang, Y.-L., and Small, J. V. 1996. Coordination of protrusion and translocation of the keratocyte involves rolling of the cell body. The Journal of Cell Biology 134:1209-1218. doi: 10.1083/jcb.134.5.1209

Andrews, P. M. 1976. Microplicae: characteristic ridge-like folds of the plasmalemma. The Journal of Cell Biology 68:420-429. doi: 10.1083/jcb.68.3.420

Arner, A. 1983. Force-velocity relation in chemically skinned rat portal vein. Pflügers Archiv-European Journal of Physiology 397:6-12. doi: 10.1007/BF00585160

Bachewich, C., and Heath, I. B. 1998. Radial F-actin arrays precede new hypha formation in Saprolegnia: implications for establishing polar growth and regulating tip morphogenesis. Journal of Cell Science 111:2005-2016.

Balinsky, B. I. 1957. New experiments on the mode of action of the limb inductor. Journal of Experimental Zoology 134:239-273.

Baluška, F., Jasik, J., Edelmann, H. G., Salajová, T., and Volkmann, D. 2001. Latrunculin B-induced plant dwarfism: plant cell elongation is F-actin-dependent. Developmental Biology 231:113-124. doi: 10.1006/dbio.2000.0115

Baluška, F., Wojtaszek, P., Volkmann, D., and Barlow, P. 2003. The architecture of polarized cell growth: the unique status of elongating plant cells. BioEssays 25:569-576. doi: 10.1002/bies.10282

Baños, F. G. D., Bordas, J., Lowy, J., and Svensson, A. 1996. Small segmental rearrangements in the myosin head can explain force generation in muscle. Biophysical Journal 71:576-589. doi: 10.1016/S0006-3495(96)79292-5

Bartels, E. M., and Elliott, G.F.1985. Donnan potentials from the A- and I-bands of glycerinated and chemically skinned muscles, relaxed and in rigor. Biophysical Journal 48:61-76. doi: 10.1016/S0006-3495(85)83760-7

Bartoo, M. L., Popov, V. I., Fearn, L. A., and Pollack, G. H. 1993. Active tension generation in isolated skeletal myofibrils. Journal of Muscle Research and Cell Motility 14:498-510. doi: 10.1007/BF00297212

Begg, D. A., Rebhun, L. I., and Hyatt, H. A. 1982. Structural organization of actin in the sea urchin egg cortex: microvillar elongation in the absence of actin filament bundle formation. The Journal of Cell Biology 93:24-32. doi: 10.1083/jcb.93.1.24

Bellairs, R. 1979. The mechanism of somite segmentation in the chick embryo. Journal of Embryology and Experimental Morphology 51:227-243.

Bereiter-Hahn, J., Lück, M., Miebach, T., Stelzer, H. K., and Vöth, M. 1990. Spreading of trypsinized cells: cytoskeletal dynamics and energy requirements. Journal of Cell Science 96:171-188.

Bereiter-Hahn, J., and Lüers, H. 1998. Subcellular tension fields and mechanical resistance of the lamella front related to the direction of locomotion. Cell Biochemistry and Biophysics 29:243-262. doi: 10.1007/BF02737897

Bereiter-Hahn, J., and Strohmeier, R. 1987. Hydrostatic pressure in metazoan cells in culture: its involvement in locomotion and shape genereation; pp. 261-272 in BereiterHahn, J., Anderson, O. R., and Reif, W-E. (eds.), Cytomechanics. The mechanical basis of cell form and structure. Springer Verlag, Berlin.

Bereiter-Hahn, J., Strohmeier, R., Kunzenbacher, I., Beck, K. and Vöth, M. 1981. Locomotion of Xenopus epidermal cells in primary culture. Journal of Cell Science 52:289-311.

Bergmann, J. E., Kupfer, A., and Singer, S. J. 1983. Membrane insertion at the leading edge of motile fibroblasts. Proceedings of the National Academy of Sciences of the United States of America 80:1367-1371.

Bernfield, M. R., Cohn, R. H., and Banerjee, S. D. 1973. Glycosaminoglycans and epithelial organ formation. American Zoologist 13:1067-1083. doi: 10.1093/icb/13.4.1067

Bialojan, C., Ruegg, J. C., and Takai, A. 1988. Effects of okadaic acid on isometric tension and myosin phosphorylation of chemically skinned guinea-pig taenia coli. The Journal of Physiology 398:81-95.

Bliokh, Z. L., Domnina, L. V., Ivanova, O. Yu., Pletjushkina, O. Yu., Svitkina, T. M., Smolyaninov, V. A., Vasiliev, J. M., and Gelfand, L. M. 1980. Spreading of fibroblasts in medium containing cytochalasin B: formation of lamellar cytoplasm as a combination of several functionally different processes. Proceedings of the National Academy of Sciences of the United States of America 77:5919-5922. 
Block, S. M. 1996. Fifty ways to love your lever: myosin motors. Cell 87:151-157. doi: 10.1016/S0092-8674(00)81332-X

Bluemink, J. G., and de Laat, S. W. 1973. New membrane formation during cytokinesis in normal and cytochalasin B-treated eggs of Xenopus laevis. I. Electron microscope observations. The Journal of Cell Biology 59:89-108. doi: 10.1083/jcb.59.1.89

Bond, M., Kitazawa, T., Somlyo, A. P., and Somlyo, A. V. 1984. Release and recycling of calcium by the sarcoplasmic reticulum in guinea-pig portal vein smooth muscle. The Journal of Physiology 355:677-695.

Borisy, G. G., and Svitkina, T. M. 2000. Actin machinery: pushing the envelope. Current Opinion in Cell Biology 12:104112. doi: 10.1016/S0955-0674(99)00063-0

Borkhvardt, V.G.1982. Morfogenez i evolyutsiya osevogo skeleta [Morphogenesis and evolution of axial skeleton]. 144 pp. Izdatelstvo Leningradskogo gosudarstvennogo universiteta, Leningrad.

Borkhvardt, V. G. 1994. Razvitie konechnostei u lichinok sibirskogo uglozuba, Salamandrella keyserlingii (Amphibia, Hynobiidae) [Development of the limbs in larvae of Siberian salamander, Salamandrella keyserlingii (Amphibia, Hynobiidae)]. Zoologicheskii Zhurnal 73:53-67.

Borkhvardt, V. G. 2000. Growth and shaping of the fin and limb buds. Russian Journal of Developmental Biology 31:154-161. doi: 10.1007/BF02758819

Borkhvardt, V. G. 2001. On the growth mechanics of the hynobiid limb bud. Russian Journal of Herpetology 8:63-67.

Borkhvardt, V.G.2002a. Gidromekhanicheskaya model formoobrazovatelnykh protsessov [Hydromechanical model of the morphogenetic processes]. Vestnik Sankt-Peterburgskogo Universiteta, Series 3. Biologia. (3):3-21.

Borkhvardt, V. G. 2002b. Invagination and evagination: a hydromechanical model. Russian Journal of Developmental Biology 33:13-21. doi: 10.1023/A:1013816927438

Borkhvardt, V. G. 2009. Mekhanicheskie preobrazovaniya zhivykh polostnykh tel [Mechanical transformations of living cavitary bodies]. 71 pp. Izdatelstvo TESSA, Saint Petersburg.

Borkhvardt, V. G. 2010. Mekhanicheskie preobrazovaniya zhivykh polostnykh tel [Mechanical transformations of living cavitary bodies]. Second Edition. 135 pp. Izdatelstvo TESSA, Saint Petersburg.

Borkhvardt, V. G. 2016. Mekhanicheskie preobrazovaniya zhivykh polostnykh tel [Mechanical transformations of living cavitary bodies]. Third Edition. 140 pp. Izdatelstvo Rossiiskogo gosudarstvennogo pedagogicheskogo universiteta imeni A. I. Gertsena, Saint Petersburg.

Borkhvardt, V.G., and Kovalenko, E. E. 1985. Rol mekhanicheskikh vzaimodeistvii $v$ razvitii miomerov i osevogo skeleta anamnii [Role of mechanical interactions in the development of myomeres and axial skeleton of anamniotes]. Vestnik Leningradskogo Universiteta, Series 3. (3):3-10.

Borkhvardt, V. G., and Kovalenko, E. E. 1986. Septalnye peregorodki embrionov i lichinok anamnii [Septa of embryos and larvae of anamniotes]. Doklady Akademii Nauk SSSR 287:764-767.

Bottino, D., Mogilner, A., Roberts, T., Stewart, M., and Oster, G. 2002. How nematode sperm crawl. Journal of Cell Science 115:367-384.

Bowers, B., and Korn, E. D. 1969. The fine structure of Acanthamoeba castellanii (Neff strain). II. Encystment. The Journal of Cell Biology 41:786-805. doi: 10.1083/jcb.41.3.786

Brawley, S. H., and Robinson, K. R. 1985. Cytochalasin treatment disrupts the endogenous currents associated with cell polarization in fucoid zygotes: studies of the role of F-actin in embryogenesis. The Journal of Cell Biology 100:1173-1184. doi: 10.1083/jcb.100.4.1173

Bray, D. 1970. Surface movements during the growth of single explanted neurons. Proceedings of the National Academy of Sciences of the United States of America 65:905-910.

Bray, D., Money, N. P., Harold, F. M., and Bamburg, J. R. 1991. Responses of growth cones to changes in osmolality of the surrounding medium. Journal of Cell Science 98:507515.

Bretscher, M. S. 1983. Distribution of receptors for transferrin and low density lipoprotein on the surface of giant HeLa cells. Proceedings of the National Academy of Sciences of the United States of America 80:454-458.

Bretscher, M. S. 1988. Fibroblasts on the move. The Journal of Cell Biology 106:235-237.

Brette, F., Calaghan, S. C., Lappin, S., White, E., Colyer, J., and Le Guennec, J.-Y. 2000.

Biphasic effects of hyposmotic challenge on excitation-contraction coupling in rat ventricular myocytes. American Journal of Physiology - Heart and Circulatory Physiology 279:H1963-H1971.

Bridgman, P.C, and Dailey, M.E.1989. The organization of myosin and actin in rapid frozen nerve growth cones. The Journal of Cell Biology 108:95-109. doi: 10.1083/ jcb.108.1.95

Brody, J. S., Vaccaro, C. A., Gill, P. J., and Silbert, J. E. 1982. Alterations in alveolar basement membranes during postnatal lung growth. The Journal of Cell Biology 95:394-402. doi: 10.1083/jcb.95.2.394

Brown, J., and Bridgman, P. C. 2003. Role of myosin II in axon outgrowth. Journal of Histochemistry and Cytochemistry 51:421-428. doi: 10.1177/002215540305100403

Brun, R. B., and Garson, J. A. 1983. Neurulation in the mexican salamander (Ambystoma mexicanum): a drug study and cell shape analysis of the epidermis and the neural plate. Journal of Embryology and Experimental Morphology 74:275-295.

Burgess, D. R., and Grey, R. D. 1974. Alterations in morphology of developing microvilli elicited by cytochalasin B. Studies of embryonic chick intestine in organ culture. The Journal of Cell Biology 62:566-575.

Burgess, D. R., and Prum, B. E. 1982. Reevaluation of brush border motility: calcium induces core filament solation and microvillar vesiculation. The Journal of Cell Biology 94:97-107.

Burnside, B. 1973. Microtubules and microfilaments in amphibian neurulation. American Zoologist 13:989-1006. doi: 10.1093/icb/13.4.989

Burnside, B., and Manasek, F.J. 1972. Cytochalasin B: problems in interpreting its effect on cells. Developmental Biology 27:443-444. doi: 10.1016/0012-1606(72)90184-4

Burridge, K.1981. Are stress fibres contractile? Nature 294:691-692. doi: 10.1038/294691a0

Butler, T. M., Narayan, S. R., Mooers, S. U., Hartshorne D. J., and Siegman M.J. 2001. The myosin cross-bridge cycle and its control by twitchin phosphorylation in catch muscle. Biophysical Journal 80:415-426. doi: 10.1016/ S0006-3495(01)76024-9

Byers, T. J., and Armstrong, P. B. 1986. Membrane protein redistribution during Xenopus first cleavage. The Journal of Cell Biology 102:2176-2184. doi: 10.1083/jcb.102.6.2176

Cabib, E., Sburlati, A., Bowers, B., and Silverman, S.J. 1989. Chitin synthase 1, an auxiliary enzyme for chitin synthesis in Saccharomyces cerevisiae. The Journal of Cell Biology 108:1665-1672. doi: 10.1083/jcb.108.5.1665

Cai, G., and Cresti, M. 2009. Organelle motility in the pollen tube: a tale of 20 years. Journal of Experimental Botany 60:495-508. doi: 10.1093/jxb/ern321 
Cala, P. M. 1980. Volume regulation by Amphiuma red blood cells. The membrane potential and its implications regarding the nature of the ion-flux pathways. The Journal of General Physiology 76:683-708.

Cameron, I., Rinaldi, R. A., Kirby, G., and Davidson, D. 2007. Amoeba proteus displays a walking form of locomotion. Cell Biology International 31:759-762. doi: 10.1016/j.cellbi.2007.01.009

Cantiello, H. F., Prat, A. G., Bonventre, J. V., Cunningham, C. C., Hartwig, J. H., and Ausiello, D. A. 1993. Actin-binding protein contributes to cell volume regulatory ion channel activation in melanoma cells. Journal of Biological Chemistry 268:4596-4599.

Carlsen, F., Knappeis, G. G., and Buchthal, F. 1961. Ultrastructure of the resting and contracted striated muscle fiber at different degrees of stretch. The Journal of Biophysical and Biochemical Cytology 11:95-117. doi: 10.1083/ jcb.11.1.95

Carlson, B.1983. Osnovy embriologii po Pettenu [Patten's Foundations of Embryology]. Vol. 1. 357 pp. Izdatelstvo Mir, Moscow.

Carton, I., Hermans, D., and Eggermont, J. 2003. Hypotonicity induces membrane protrusions and actin remodeling via activation of small GTPases Rac and Cdc42 in Rat-1 fibroblasts. American Journal of Physiology - Cell Physiology 285:C935-C944. doi: 10.1152/ajpcell.00069.2003

Chambers, C., and Grey, R.D.1979. Development of the structural components of the brush border in absorptive cells of the chick intestine. Cell and Tissue Research 204:387-405. doi: 10.1007/BF00233651

Chandler, D. E., and Heuser, J. 1981. Postfertilization growth of microvilli in the sea urchin egg: new views from eggs that have been quick-frozen, freeze-fractured, and deeply etched. Developmental Biology 82:393-400. doi: 10.1016/0012-1606(81)90463-2

Charras, G. T., Hu, C.-K., Coughlin, M., and Mitchison, T. J. 2006. Reassembly of contractile actin cortex in cell blebs. The Journal of Cell Biology 175:477-490. doi: 10.1083/ jcb.200602085

Charras, G. T., Yarrow, J. C., Horton, M. A., Mahadevan, L., and Mitchison, T.J. 2005. Non-equilibration of hydrostatic pressure in blebbing cells. Nature 435:365-369. doi: 10.1038/nature03550

Chaumont, F., Barrieu, F., Herman, E. M., and Chrispeels, M. J. 1998. Characterization of a maize tonoplast aquaporin expressed in zones of cell division and elongation. Plant Physiology 117:1143-1152. doi: 10.1104/ pp.117.4.1143

Chebli, Y., and Geitmann, A. 2007. Mechanical principles governing pollen tube growth. Functional Plant Science and Biotechnology 1:232-245.

Chen, W.-T.1981. Mechanism of retraction of the trailing edge during fibroblast movement. The Journal of Cell Biology 90:187-200. doi: 10.1083/jcb.90.1.187

Chrispeels, M. J., and Maurel, C. 1994. Aquaporins: the molecular basis of facilitated water movement through living plant cells? Plant Physiology 105:9-13.

Colas, J.-F., and Schoenwolf, G. C. 2001. Towards a cellular and molecular understanding of neurulation. Developmental Dynamics 221:117-145. doi: 10.1002/dvdy.1144

Cole, R. A., and Fowler, J.E. 2006. Polarized growth: maintaining focus on the tip. Current Opinion in Plant Biology 9:579-88. doi: 10.1016/j.pbi.2006.09.014

Colomo, F., Piroddi, N., Poggesi, C., te Kronnie, G., and Tesi, C. 1997. Active and passive forces of isolated myofibrils from cardiac and fast skeletal muscle of the frog. The Journal of Physiology 500:535-548. doi: 10.1113/jphysiol.1997.sp022039
Conti, M. A., and Adelstein, R. S. 2008. Nonmuscle myosin II moves in new directions. Journal of Cell Science 121:1118. doi: $10.1242 /$ jcs.007112

Cooke, R. 2004. The sliding filament model: 1972-2004. The Journal of General Physiology 123:643-656. doi: 10.1085/ jgp.200409089

Cooper, J.A. 1987. Effects of cytochalasin and phalloidin on actin. The Journal of Cell Biology 105:1473-1478. doi: 10.1083/jcb.105.4.1473

Cornet, M., Lambert, I. H., and Hoffmann, E. K. 1993. Relation between cytoskeleton, hypo-osmotic treatment and volume regulation in Ehrlich ascites tumor cells. The Journal of Membrane Biology 131:55-66. doi: 10.1007/ BF02258534

Cosgrove, D. J. 1997. Relaxation in a high-stress environment: the molecular bases of extensible cell walls and cell enlargement. The Plant Cell 9:1031-1041. doi: 10.1105/ tpc.9.7.1031

Cunningham, C. C. 1995. Actin polymerization and intracellular solvent flow in cell surface blebbing. The Journal of Cell Biology 129:1589-1599. doi: 10.1083/jcb.129.6.1589

Dabagyan, N. V., and Sleptsova, L. A. 1975. Travyanaya lyagushka Rana temporaria L. [Common frog Rana temporaria L. ]; pp. 442-462 in Dettlaff, T. A. (ed.), Objects of Developmental Biology. Izdatelstvo Nauka, Moscow.

Dai, J., Sheetz, M.P., Wan, X., and Morris, C. E. 1998. Membrane tension in swelling and shrinking molluscan neurons. The Journal of Neuroscience 18:6681-6692.

Dan, K., and Dan, J. C. 1947. Behavior of the cell surface during cleavage. VIII. On the cleavage of medusan eggs. The Biological Bulletin 93:163-188. doi: 10.2307/1538286

Dan, K., and Okazaki, K. 1956. Cyto-embryological studies of sea urchins. III. Role of the secondary mesenchyme cells in the formation of the primitive gut in sea urchin larvae. The Biological Bulletin 110:29-42. doi: 10.2307/1538890

Danilchik, M. V., Bedrick, S. D., Brown, E. E., and Ray, K. 2003. Furrow microtubules and localized exocytosis in cleaving Xenopus laevis embryos. Journal of Cell Science 116:273283. doi: 10.1242/jcs.00217

Davidson, L. A. 2012. No strings attached: new insights into epithelial morphogenesis. BMC Biology 10:105-108. doi: 10.1186/1741-7007-10-105

Davidson, L. A., Koehl, M. A. R., Keller, R., and Oster, G. F. 1995. How do sea urchins invaginate? Using biomechanics to distinguish between mechanisms of primary invagination. Development 121:2005-2018.

Davidson, L. A., Oster, G. F., Keller, R. E., and Koehl, M.A.R. 1999. Measurements of mechanical properties of the blastula wall reveal which hypothesized mechanisms of primary invagination are physically plausible in the sea urchin Strongylocentrotus purpuratus. Developmental Biology 209:221-238. doi: 10.1006/dbio.1999.9249

DeBiasio, R. L., Wang, L.-L., Fisher, G. W., and Taylor, D. L. 1988. The dynamic distribution of fluorescent analogues of actin and myosin in protrusions at the leading edge of migrating Swiss 3T3 fibroblasts. The Journal of Cell Biology 107:2631-2645. doi: 10.1083/jcb.107.6.2631

Dembo, M. 1989. Mechanics and control of the cytoskeleton in Amoeba proteus. Biophysical Journal 55:1053-1080. doi: 10.1016/S0006-3495(89)82904-2

Devine, C. E, Somlyo, A. V, and Somlyo, A. P. 1972. Sarcoplasmic reticulum and excitation-contraction coupling in mammalian smooth muscles. The Journal of Cell Biology 52:690-718. doi: 10.1083/jcb.52.3.690

Dillon, P. F., and Murphy, R. A. 1982. High force development and crossbridge attachment in smooth muscle from swine carotid arteries. Circulation Research 50:799-804. doi: 10.1161/01.RES. 50.6.799 
Draeger, A., Amos, W. B., Ikebe, M., and Small, J. V. 1990. The cytoskeletal and contractile apparatus of smooth muscle: contraction bands and segmentation of the contractile elements. The Journal of Cell Biology 111:2463-2473. doi: $10.1083 / j c b .111 .6 .2463$

Dydyńska, M., and Wilkie, D. R. 1963. The osmotic properties of striated muscle fibres in hypertonic solutions. With an appendix by H. E. Huxley, Sally Page and D. R. Wilkie. The Journal of Physiology 169:312-329. doi: 10.1113/jphysiol.1963.sp007258

Eddy, R.J., Pierini, L. M., Matsumura, F., and Maxfield, F. R. 2000. $\mathrm{Ca}^{2+}$-dependent myosin II activation is required for uropod retraction during neutrophil migration. Journal of Cell Science 113:1287-1298.

Ede, D. A., Bellairs, R., and Bancroft, M. 1974. A scanning electron microscope study of the early limb-bud in normal and talpid3 mutant chick embryos. Journal of Embryology and Experimental Morphology 31:761-785.

Ede, D. A., and Law, J.T.1969. Computer simulation of vertebrate limb morphogenesis. Nature 221:244-248. doi: 10.1038/221244a0

Edwards, G. A., Ruska, H., Souza, S.P., and Vallejo-Freire, A. 1956. Comparative cytophysiology of striated muscle with special reference to the role of the endoplasmic reticulum. The Journal of Biophysical and Biochemical Cytology 2:143-156. doi: 10.1083/jcb.2.4.143

Elliott, G. F. 1973. Donnan and osmotic effects in muscle fibers without membranes. Journal of Mechanochemistry and Cell Motility 2:83-89.

Elliott, G. F., Rome, E. M., and Spencer, M.1970. A type of contraction hypothesis applicable to all muscles. Nature 226:417-420. doi: 10.1038/226417a0

Engel, A., Fujiyoshi, Y., and Agre, P. 2000. The importance of aquaporin water channel protein structures. The EMBO Journal 19:800-806. doi: 10.1093/emboj/ 19.5.800

Engqvist-Goldstein, Å.E. Y., and Drubin, D. G. 2003. Actin assembly and endocytosis: from yeast to mammals. Annual Review of Cell and Developmental Biology 19:287-332. doi: 10.1146/annurev.cellbio.19.111401.093127

Ettensohn, C.A.1984. Primary invagination of the vegetal plate during sea urchin gastrulation. American Zoologist 24:571-588. doi: 10.1093/icb/24.3.571

Ettensohn, C. A. 1985a. Gastrulation in the sea urchin embryo is accompanied by the rearrangement of invaginating epithelial cells. Developmental Biology 112:383-390. doi: 10.1016/0012-1606(85)90410-5

Ettensohn, C.A.1985b. Mechanisms of epithelial invagination. The Quarterly Review of Biology 60:289-307. doi: $10.1086 / 414426$

Eveloff, J. L., and Warnock, D. G. 1987. Activation of ion transport systems during cell volume regulation. American Journal of Physiology - Renal Physiology 252:F1-F10.

Even-Ram, S., Doyle, A. D., Conti, M. A., Matsumoto, K., Adelstein, R. S., and Yamada, K. M. 2007. Myosin IIA regulates cell motility and actomyosin-microtubule crosstalk. $\mathrm{Na}$ ture Cell Biology 9:299-309. doi: 10.1038/ncb1540

Everhart, L. P., JR., and Rubin, R. W. 1974. Cyclic changes in the cell surface. II. The effect of cytochalasin B on the surface morphology of synchronized chinese hamster ovary cells. The Journal of Cell Biology 60:442-447.

Fackler, O.T., and Grosse, R. 2008. Cell motility through plasma membrane blebbing. The Journal of Cell Biology 181:879-884. doi: 10.1083/jcb.200802081

Fay, F. S., and Delise, C.M.1973. Contraction of isolated smooth-muscle cells - structural changes. Proceedings of the National Academy of Sciences of the United States of America 70:641-645.
Fedier, A., and Keller, H. U. 1997. Suppression of bleb formation, locomotion, and polarity of walker carcinosarcoma cells by hypertonic media correlates with cell volume reduction but not with changes in the F-actin content. Cell Motility and the Cytoskeleton 37:326-337. doi: 10.1002/ (SICI)1097-0169(1997)37:4<326::AID-CM4>3.0.CO;2-2

Fenteany, G., and Zhu, S. 2003. Small-molecule inhibitors of actin dynamics and cell motility. Current Topics in Medicinal Chemistry 3:593-616. doi: 10.2174/1568026033452348

Fischer-Parton, S., Parton, R. M., Hickey, P.C., Dijksterhuis, J., Atkinson, H.A., and Read, N.D. 2000. Confocal microscopy of FM4-64 as a tool for analyzing endocytosis and vesicle trafficking in living fungal hyphae. Journal of Microscopy 198:246-259. doi: 10.1046/j.13652818.2000.00708.x

Fisher, B.A., and Bagby, R. M. 1977. Reorientation of myofilaments during contraction of a vertebrate smooth muscle. American Journal of Physiology - Cell Physiology 232:C5-C14.

Fishkind, D. J., and Wang, Y.-L. 1993. Orientation and threedimensional organization of actin filaments in dividing cultured cells. The Journal of Cell Biology 123:837-848. doi: 10.1083/jcb.123.4.837

Flanagan, L. A., Chou, J., Falet, H., Neujahr, R., Hartwig, J. H., and Stossel, T.P. 2001. Filamin A, the Arp2/3 complex, and the morphology and function of cortical actin filaments in human melanoma cells. The Journal of Cell Biology 155:511-517. doi: 10.1083/jcb.200105148

Forscher, P., Kaczmarek, L. K., Buchanan, J., and Smith, S.J. 1987. Cyclic AMP induces changes in distribution and transport of organelles within growth cones of Aplysia bag cell neurons. The Journal of Neuroscience 7:3600-3611.

Friedl, P., Borgmann, S., and Bröcker, E. B. 2001. Amoeboid leukocyte crawling through extracellular matrix: lessons from the Dictyostelium paradigm of cell movement. Journal of Leukocyte Biology 70:491-509.

Friedman, A. L., and Goldman, Y. E. 1996. Mechanical characterization of skeletal muscle myofibrils. Biophysical Journal 71:2774-2785. doi: 10.1016/S0006-3495(96)79470-5

Fukui, Y., De Lozanne, A., and Spudich, J. A. 1990. Structure and function of the cytoskeleton of a Dictyostelium myosin-defective mutant. The Journal of Cell Biology 110:367378. doi: 10.1083/jcb.110.2.367

Fukui, Y., Kitanishi-Yumura, T., and Yumura, S. 1999. Myosin II-independent F-actin flow contributes to cell locomotion in Dictyostelium. Journal of Cell Science 112:877-886.

Gallagher, B.C.1986. Basal laminar thinning in branching morphogenesis of the chick lung as demonstrated by lectin probes. Journal of Embryology and Experimental Morphology 94:173-178.

Geeves, M. A. 1991.The dynamics of actin and myosin association and the crossbridge model of muscle contraction. Biochemical Journal 274:1-14. doi: 10.1042/bj2740001

Gillis, J. M., Cao, M. L., and Godfraind-De Becker, A. 1988. Density of myosin filaments in the rat anococcygeus muscle, at rest and in contraction. II. Journal of Muscle Research and Cell Motility 9: 18-28. doi: 10.1007/BF01682145

Ginsburg, A.S., and Dettlaff, T.A. Osyotr Acipenser güldenstädti [Sturgeon Acipenser güldenstädti]; pp. 217-263 in Dettlaff, T.A. (ed.), Objects of Developmental Biology. Izdatelstvo Nauka, Moscow.

Glaesner, L. 1925. Normentafeln zur Entwicklungsgeschichte des gemeinen Wassermolches (Molge vulgaris). 49 SS. Fisher Verlag, Jena.

Glück, E., and Paul, R.J.1977. The aerobic metabolism of porcine carotid artery and its relationship to isometric force. Pflügers Archiv - European Journal of Physiology 370:9-18. doi: 10.1007/BF00707939 
Godman, G. C., Miranda, A. F., Deitch, A. D., and Tanenbaum, S.W. 1975. Action of cytochalasin D on cells of established lines. III. Zeiosis and movements at the cell surface. The Journal of Cell Biology 64:644-667. doi: 10.1083/ jcb.64.3.644

Godt, R. E. 1981. A simple electrostatic model can explain the effect of $\mathrm{pH}$ upon the force-pCa relation of skinned frog skeletal muscle fibres. Biophysical Journal 35:385-392. doi: 10.1016/S0006-3495(81)84797-2

Godt, R. E, and Maughan, D.W. 1977. Swelling of skinned muscle fibers of the frog. Experimental observations. Biophysical Journal 19:103-116. doi: 10.1016/S00063495(77)85573-2

Goldman, Y. E., and Simmons R. M. 1986. The stiffness of frog skinned muscle fibres at altered lateral filament spacing. The Journal of Physiology 378:175-194. doi: 10.1113/ jphysiol.1986.sp016213

Gordon, A. M., Godt, R.E., Donaldson, S. K. B., and Harris, C. E. 1973. Tension in skinned frog muscle fibers in solutions of varying ionic strength and neutral salt composition. The Journal of General Physiology 62:550-574. doi: 10.1085/jgp.62.5.550

Gordon, R. 1985. A review of the theories of vertebrate neurulation and their relationship to the mechanics of neural tube birth defects. Journal of Embryology and Experimental Morphology 89. Suppl.:229-255.

Gould, R. P., Day, A., and Wolpert, L. 1972. Mesenchymal condensation and cell contact in early morphogenesis of the chick limb. Experimental Cell Research 72:325-336. doi: 10.1016/0014-4827(72)90593-9

Gradmann, D., and Robinson, D. G. 1989. Does turgor prevent endocytosis in plant cells? Plant Cell and Environment 12:151-154. doi: 10.1111/j.1365-3040.1989.tb01927.x

Gray, D.I., Gooday, G.W., and Prosser, J.l.1990. Apical hyphal extension in Streptomyces coelicolor A3(2). Journal of General Microbiology 136:1077-1084. doi: 10.1099/00221287-136-6-1077

Grębecka, L., and Grębecki, A. 1975. Morphometric study of moving Amoeba proteus. Acta Protozoologica 14:337-361. doi: 10.1099/00221287-136-6-1077

Grębecki, A. 1984. Relative motion in Amoeba proteus in respect to the adhesion sites. I. Behavior of monotactic forms and the mechanism of fountain phenomenon. Protoplasma 123:116-134.

Grębecki, A. 1990. Dynamics of the contractile system in the pseudopodial tips of normally locomoting amoebae, demonstrated in vivo by video-enhancement. Protoplasma 154:98-111. doi: 10.1007/BF01539837

Grębecki, A. 1991. Participation of the contractile system in endocytosis demonstrated in vivo by video-enhancement in heart-pretreated amoebae. Protoplasma 160:144-158. doi: 10.1007/BF01539966

Grębecki, A. 1994. Membrane and cytoskeleton flow in motile cells with emphasis on the contribution of free-living amoebae. International Review of Cytology 148:37-80. doi: 10.1016/S0074-7696(08)62405-5

Grimm, H. P., Verkhovsky, A. B., Mogilner, A., and Meister, J.J. 2003. Analysis of actin dynamics at the leading edge of crawling cells: implications for the shape of keratocyte lamellipodia. European Biophysical Journal 32:563-577. doi: 10.1007/s00249-003-0300-4

Grinstein, S., Rothstein, A., Sarkadi, B., and Gelfand, E. W. 1984. Responses of lymphocytes to anisotonic media: volumeregulating behavior. American Journal of Physiology - Cell Physiology 246:C204-C215.

Grobstein, C., and Cohen, J. 1965. Collagenase: effect on the morphogenesis of embryonic salivary epithelium in vitro. Science 150:626-628. doi: 10.1126/science.150.3696.626
Gross, S.P., Tuma, M. C., Deacon, S. W., Serpinskaya, A. S., Reilein, A.R., and Gelfand, V.I. 2002. Interactions and regulation of molecular motors in Xenopus melanophores. The Journal of Cell Biology 156:855-865. doi: 10.1083/jcb.200105055

Guilford, W. H., Dupuis, D. E., Kennedy, G., Wu, J., Patlak, J. B. and Warshaw, D.M.1997. Smooth muscle and skeletal muscle myosins produce similar unitary forces and displacements in the laser trap. Biophysical Journal 72:1006-1021. doi: 10.1016/S0006-3495(97)78753-8

Gunst, S. J., and Fredberg, J.J. 2003. The first three minutes: smooth muscle contraction, cytoskeletal events, and soft glasses. Journal of Applied Physiology 95:413-425. doi: 10.1152/japplphysiol.00277.2003

Haddock, R.E, and Hill, C.E. 2005. Rhythmicity in arterial smooth muscle. The Journal of Physiology 566:645-656. doi: 10.1113/jphysiol.2005.086405

Hanson, J. 1952. Changes in the cross-striation of myofibrils during contraction induced by adenosine triphosphate. Nature 169:530-533. doi: 10.1038/169530a0

Hara-Chikuma, M., and Verkman, A. S. 2006. Aquaporin-1 facilitates epithelial cell migration in kidney proximal tubule. Journal of the American Society of Nephrology 17:3945. doi: 10.1681/ASN. 2005080846

Hardin, J. 1988. The role of secondary mesenchyme cells during sea urchin gastrulation studied by laser ablation. Development 103:317-324.

Hardin, J. 1989. Local shifts in position and polarized motility drive cell rearrangement during sea urchin gastrulation. Developmental Biology 136:430-445. doi: 10.1016/00121606(89)90268-6

Hardin, J. D., and Cheng, L. Y. 1986. The mechanisms and mechanics of archenteron elongation during sea urchin gastrulation. Developmental Biology 115:490-501. doi: 10.1016/0012-1606(86)90269-1

Harold, F. M. 1990. To shape a cell: an inquiry into the causes of morphogenesis of microorganisms. Microbiology Reviews 54:381-431.

Harold, F. M. 2002. Force and compliance: rethinking morphogenesis in walled cells. Fungal Genetics and Biology 37:271-282. doi: 10.1016/S1087-1845(02)00528-5

Harold, R. L., Money, N. P., and Harold, F. M. 1996. Growth and morphogenesis in Saprolegnia ferax: is turgor required? Protoplasma 191:105-114. doi: 10.1007/BF01280830

Harrington, W. F. 1979. On the origin of the contractile force in skeletal muscle. Proceedings of the National Academy of Sciences of the United States of America 76:5066-5070.

Heidemann, S. R., Lamoureux, P., and Buxbaum, R. E. 1990. Growth cone behavior and production of traction force. The Journal of Cell Biology 111:1949-1957. doi: 10.1083/ jcb.111.5.1949

Hepler, P. K., Vidali, L., and Cheung, A. Y. 2001. Polarized cell growth in higher plants. Annual Review of Cell and Developmental Biology 17:159-187. doi: 10.1146/annurev.cellbio.17.1.159

Hering, H., and Sheng, M. 2001. Dendritic spines: structure, dynamics and regulation. Nature Reviews Neuroscience 2:880-888. doi: 10.1038/35104061

Hermann, G. J., and Shaw, J. M. 1998. Mitochondrial dynamics in yeast. Annual Review of Cell and Developmental Biology 14:265-303. doi: 10.1146/annurev.cellbio.14.1.265

Herrera, A. M., McParland, B. E., Bienkowska, A., Tait, R., Paré, P.D., and Seow, C.Y.2005. 'Sarcomeres' of smooth muscle: functional characteristics and ultrastructural evidence. Journal of Cell Science 118:2381-2392. doi: $10.1242 / \mathrm{jcs} .02368$

Heuser, J. 1989. Effects of cytoplasmic acidification on clathrin lattice morphology. The Journal of Cell Biology 108:401411. doi: 10.1083/jcb.108.2.401 
Hiramoto, Y. 1981. Mechanical properties of dividing cells; pp. 397-418 in Zimmerman, A. M. (ed.), Mitosis, Cytokinesis. Academic Press, New York, London.

Hirokawa, N. 1982. Cross-linker system between neurofilaments, microtubules, and membranous organelles in frog axons revealed by the quick-freeze, deep-etching method. The Journal of Cell Biology 94:129-142. doi: 10.1083/jcb.94.1.129

Hirokawa, N., Glicksman, M. A., and Willard, M. B. 1984. Organization of mammalian neurofilament polypeptides within the neuronal cytoskeleton. The Journal of Cell Biology 98:1523-1536. doi: 10.1083/jcb.98.4.1523

Hirokawa, N., and Heuser, J. E. 1981. Quick-freeze, deep-etch visualization of the cytoskeleton beneath surface differentiations of intestinal epithelial cells. The Journal of Cell Biology 91:399-409. doi: 10.1083/jcb.91.2.399

Hirokawa, N., and Takemura, R. 2005. Molecular motors and mechanisms of directional transport in neurons. Nature Reviews Neuroscience 6:201-214. doi: 10.1038/nrn1624

Hirokawa, N., Tilney, L. G., Fujiwara, K., and Heuser, J. E. 1982. Organization of actin, myosin, and intermediate filaments in the brush border of intestinal epithelial cells. The Journal of Cell Biology 94:425-443. doi: 10.1083/ jcb.94.2.425

Hoffmann, E. K., Lambert, I. H., and Pedersen, S. F. 2009. Physiology of cell volume regulation in vertebrates. Physiological Reviews 89:193-277. doi: 10.1152/physrev.00037.2007

Holdaway-Clarke, T. L, Feijó, J.A, Hackett, G. R, Kunkel, J. G, and Hepler, P. K. 1997. Pollen tube growth and intracellular cytosolic calcium gradient oscillate in phase while extracellular calcium influx is delayed. The Plant Cell 9:1999-2010. doi: 10.1105/tpc.9.11.1999

Hollenbeck, P. J., and Saxto, W. M. 2005. The axonal transport of mitochondria. Journal of Cell Science 118:5411-5419. doi: $10.1242 /$ jcs. 02745

Holtzer, H., and Sanger, J. W. 1972. Cytochalasin-B: Microfilaments, cell movement and what else? Developmental Biology 27:444-446.

Höner, B., Citi, S., Kendrick-Jones, J., and Jockusch, B. M. 1988. Modulation of cellular morphology and locomotory activity by antibodies against myosin. The Journal of Cell Biology 107:2181-2189. doi: 10.1083/jcb.107.6.2181

Hotulainen, P., and Lappalainen, P. 2006. Stress fibers are generated by two distinct actin assembly mechanisms in motile cells. The Journal of Cell Biology 173:383-394. doi: $10.1083 /$ jcb.200511093

Houdusse, A., and Sweeney, H. L. 2001. Myosin motors: missing structures and hidden springs. Current Opinion in Structural Biology 11:182-194. doi: 10.1016/S0959440X(00)00188-3

Hsu, L. S., and Becker, E. L. 1975. Volume changes induced in rabbit polymorphonuclear leukocytes by chemotactic factor and cytochalasin B. The American Journal of Pathology 81:1-14.

Hülsmann, N., and Grębecki, A. 1995. Induction of lobopodia and lamellipodia in a filopodial organism (Vampyrella lateritia). European Journal of Protistology 31:182-189. doi: 10.1016/S0932-4739(11)80442-6

Huxley, A. F. 2000. Cross-bridge action: present views, prospects, and unknowns. Journal of Biomechanics 33:11891195. doi: 10.1016/S0021-9290(00)00060-9

Huxley, H.E.1969. The mechanism of muscular contraction. Science 164:1356-1366. doi: 10.1126/science.164.3886.1356

Huxley, H.E. 2004. Fifty years of muscle and the sliding filament hypothesis. European Journal of Biochemistry 271:1403-1415. doi: 10.1111/j.1432-1033.2004.04044.x
Irving, T. C., Konhilas, J., Perry, D., Fischetti, R., and de Tombe, P.P. 2000. Myofilament lattice spacing as a function of sarcomere length in isolated rat myocardium. American Journal of Physiology - Heart and Circulatory Physiology 279:H2568-H2573.

Isenberg, G., Rathke, P. C., Hülsmann, N., Franke, W. W., and Wohlfarth-Bottermann, K. E. 1976. Cytoplasmic actomyosin fibrils in tissue culture cells. Direct proof of contractility by visualisation of ATP-induced contraction in fibrils isolated by laser microbeam dissection. Cell and Tissue Researc 166:427-443. doi: 10.1007/BF00225909

Ivanov, I. I. 1950. Khimicheskaya dinamika myshts i podvizhnykh kletok [Chemical dynamics of muscles and motile cells]. 254 pp. Izdatelstvo Medgiz, Moscow.

Ives, H.E., Schultz, G.S., Galardy, R.E., and Jamieson, J. D. 1978. Preparation of functional smooth muscle cells from the rabbit aorta. The Journal of Experimental Medicine 148:1400-1413. doi: 10.1084/jem.148.5.1400

Iwadate, Y., and Yumura, S. 2008. Actin-based propulsive forces and myosin-II-based contractile forces in migrating Dictyostelium cells. Journal of Cell Science 121:1314-1324. doi: $10.1242 /$ jcs.021576

Izzard, C. S., and Izzard, S. L. 1975. Calcium regulation of the contractile state of isolated mammalian fibroblast cytoplasm. Journal of Cell Science 18:241-256.

Jackson, S. L., and Heath, I. B. 1990. Evidence that actin reinforces the extensible hyphal apex of the oomycete Saprolegnia ferax. Protoplasma 157:144-153. doi: 10.1007/ BF01322647

Jacobs, J. R., and Stevens, J. K. 1986. Changes in the organization of the neuritic cytoskeleton during nerve growth factor-activated differentiation of PC12 cells: a serial electron microscopic study of the development and control of neurite shape. The Journal of Cell Biology 103:895906. doi: 10.1083/jcb.103.3.895

Janmey, P. A. 1998. The cytoskeleton and cell signaling: component localization and mechanical coupling. Physiological Reviews 78:763-781.

Jay, P. Y., Pham, P. A., Wong, S. A., and Elson, E. L. 1995. A mechanical function of myosin II in cell motility. Journal of Cell Science 108:387-393.

Jurand, A. 1965. Ultrastructural aspects of early development of the fore-limb buds in the chick and the mouse. Proceedings of the Royal Society of London B 162:387-405. doi: 10.1098/rspb.1965.0045

Kaksonen, M., Toret, C. P., and Drubin, D. G. 2006. Harnessing actin dynamics for clathrin-mediated endocytosis. Nature Reviews Molecular Cell Biology 7:404-414. doi: 10.1038/nrm1940

Kanada, M., Nagasaki, A., and Uyeda, T. Q. P. 2005. Adhesiondependent and contractile ring-independent equatorial furrowing during cytokinesis in mammalian cells. Molecular Biology of the Cell 16:3865-3872. doi: 10.1091/mbc. E05-03-0233

Karfunkel, P.1974. The mechanisms of neural tube formation. International Review of Cytology 38:245-271. doi: 10.1016/S0074-7696(08)60927-4

Kargacin, G.J., Cooke, P.H., Abramson, S. B., and Fay, F.S. 1989. Periodic organization of the contractile apparatus in smooth muscle revealed by the motion of dense bodies in single cells. The Journal of Cell Biology 108:1465-1475. doi: 10.1083/jcb.108.4.1465

Kargacin, G. J., and Fay, F. S. 1987. Physiological and structural properties of saponin-skinned single smooth muscle cells. The Journal of General Physiology 90:49-73. doi: 10.1085/jgp.90.1.49

Kasai, Y., lino, M., Tsutsumi, O., Taketani, Y., and Endo, M. 1994. Effects of cyclopiazonic acid on rhythmic con- 
tractions in uterine smooth muscle bundles of the rat. British Journal of Pharmacology 112:1132-1136. doi: 10.1111/j.1476-5381.1994.tb13201.x

Katoh, K., Kano, Y., Amano, M., Kaibuchi, K., and Fujiwara, K. 2001. Stress fiber organization regulated by MLCK and Rho-kinase in cultured human fibroblasts. American Journal of Physiology - Cell Physiology 280:C1669-C1679.

Katoh, K., Kano, Y., Masuda, M., Onishi, H., and Fujiwara, K. 1998. Isolation and contraction of the stress fiber. Molecular Biology of the Cell 9:1919-1938. doi: 10.1091/ mbc.9.7.1919

Keller, H., and Eggli, P. 1998. Protrusive activity, cytoplasmic compartmentalization, and restriction rings in locomoting blebbing Walker carcinosarcoma cells are related to detachment of cortical actin from the plasma membrane. Cell Motility and the Cytoskeleton 41:181-193. doi: 10.1002/ (SICI)1097-0169(1998)41:2<181::AID-CM8>3.0.CO;2-H

Keller, R., Davidson, L. A., and Shook, D. R. 2003. How we are shaped: The biomechanics of gastrulation. Differentiation 71:171-205. doi: 10.1046/j.1432-0436.2003.710301.x

Kelley, R. O., and Bluemink, J. G. 1974. An ultrastructural analysis of cells and matrix differentiation during early limb development in Xenopus laevis. Developmental Biology 37:1-17. doi: 10.1016/0012-1606(74)90164-X

Kimberly, E. L., and Hardin, J. 1998. Bottle cells are required for the initiation of primary invagination in the sea urchin embryo. Developmental Biology 204:235-250. doi: 10.1006/dbio.1998.9075

King, L. S., Yasui, M., and Agre, P. 2000. Aquaporins in health and disease. Molecular Medicine Today 6:60-65.

Kłopocka, W., Kołodziejczyk, J., Lopatowska, A., and Grębecki, A. 1996. Relationship between pinocytosis and adhesion in Amoeba proteus. Cell Biology International 20:635-641. doi: 10.1006/cbir.1996.0083

Knecht, D. A., and Loomis, W. F. 1987. Antisense RNA inactivation of myosin heavy chain gene expression in Dictyostelium discoideum. Science 236:1081-1086.

Koch, A. L. 1990. Growth and form of the bacteria cell wall. American Scientist 18:327-341.

Koch, J.-P., and Korbmacher, C. 2000. Mechanism of shrinkage activation of nonselective cation channels in $\mathrm{M}-1$ mouse cortical collecting duct cells. The Journal of Membrane Biology 177:231-242. doi: 10.1007/s002320010006

Kolega J. 1986. The cellular basis of epithelial morphogenesis; pp. 103-143 in Browder, L. W. (ed.), Developmental biology. Vol. 2. The cellular basis of morphogenesis. Plenum Press, New York, London.

Kolega, J. 2006. The role of myosin II motor activity in distributing myosin asymmetrically and coupling protrusive activity to cell translocation. Molecular Biology of the Cell 17:4435-4445. doi: 10.1091/mbc.E06-05-0431

Komarov, V. L. 1949. Vvedenie v botaniku [Introduction into Botany]. 322 p. Izdatelstvo Sovetskaya Nauka, Moscow.

Kominami, T., and Takata, H. 2004. Gastrulation in the sea urchin embryo: A model system for analyzing the morphogenesis of a monolayered epithelium. Development, Growth and Differentiation 46:309-326. doi: 10.1111/j.1440-169x.2004.00755.x

Koshy, S. S., Montrose, M. H., and Sears, C. L. 1996. Human intestinal epithelial cells swell and demonstrate actin earrangement in response to the metalloprotease toxin of Bacteroides fragilis. Infection and Immunity 64:5022-5028.

Kossmann, T., Fürst, D., and Small, J. V. 1987. Structural and biochemical analysis of skinned smooth muscle preparations. Journal of Muscle Research and Cell Motility 8:135144. doi: 10.1007/BF01753989

Kraus, Y., and Technau, U.2006. Gastrulation in the sea anemone Nematostella vectensis occurs by invagination and immigration: an ultrastructural study. Development Genes and Evolutio 216:119-132. doi: 10.1007/s00427005-0038-3

Krishan, A. 1972. Cytochalasin-B: time-lapse cinematographic studies on its effects on cytokinesis. The Journal of Cell Biology 54:657-664. doi: 10.1083/jcb.54.3.657

Kuo, K.-H., and Seow, C. Y. 2004. Contractile filament architecture and force transmission in swine airway smooth muscle. Journal of Cell Science 117:1503-1511. doi: $10.1242 / j c s .00996$

Lampinen, M. J., and Noponen, T. 2005. Electric dipole theory and thermodynamics of actomyosin molecular motor in muscle contraction. Journal of Theoretical Biology 236:397-421. doi: 10.1016/j.jtbi.2005.03.020

Lang, F., Busch, G. L., Ritter, M., Völkl, H., Waldegger, S., Gulbins, E., and Häussinger, D. 1998. Functional significance of cell volume regulatory mechanisms. Physiological Reviews 78:247-306.

Langridge P.D., Kay R. R. 2006. Blebbing of Dictyostelium cells in response to chemoattractant. Experimental Cell Research 312: 2009-2017. doi: 10.1016/j.yexcr. 2006.03.007

Le Clainche, C., and Carlier, M.-F. 2008. Regulation of actin assembly associated with protrusion and adhesion in cell migration. Physiological Reviews 88:489-513. doi: 10.1152/physrev.00021.2007

Lee, J.-Y., and Harland, R. M. 2007. Actomyosin contractility and microtubules drive apical constriction in Xenopus bottle cells. Developmental Biology 311:40-52. doi: 10.1016/j.ydbio.2007.08.010

Lew, R. R. 2005. Mass flow and pressure-driven hyphal extension in Neurospora crassa. Microbiology 151:2685-2692. doi: 10.1099/mic.0.27947-0

Lewis, A. K, and Bridgman, P. C. 1992. Nerve growth cone lamellipodia contain two populations of actin filaments that differ in organization and polarity. The Journal of Cell Biology 119:1219-1243. doi: 10.1083/jcb.119.5.1219

Lionne, C., Iorga, B., Candau, R., and Travers, F. 2003. Why choose myofibrils to study muscle myosin ATPase? Journal of Muscle Research and Cell Motility 24:139-148. doi: 10.1023/A:1026045328949

Lipton, B. H., and Jacobson, A. G. 1974. Analysis of normal somite development. Developmental Biology 38:73-90. doi: 10.1016/0012-1606(74)90260-7

Lo, C.-M., Buxton, D. B., Chua, G. C. H., Dembo, M., Adelstein, R. S., and Wang, Y.-L. 2004. Nonmuscle myosin IIB is involved in the guidance of fibroblast migration. Molecular Biology of the Cell 15:982-989. doi: 10.1091/mbc.E03-060359

Loike, J. D., Cao, L., Kuang, K., Vera, J. C., Silverstein, S. C., and Fischbarg, J. 1993. Role of facilitative glucose transporters in diffusional water permeability through $\mathbf{7 7 4}$ cells. The Journal of General Physiology 102:897-906. doi: 10.1085/jgp.102.5.897

Loitto, V. M., Forslund, T., Sundqvist, T., Magnusson, K.-E., and Gustafsson, M. 2002. Neutrophil leukocyte motility requires directed water influx. Journal of Leukocyte Biology 71:212-222.

Loitto, V. M., Huang, C., Sigal, Y.J., and Jacobson, K. 2007. Filopodia are induced by aquaporin-9 expression. Experimental Cell Research 313:1295-1306. doi: 10.1016/j. yexcr.2007.01.023

Lopéz-Franco, R., Bartnicki-García, S., and Bracker, C. E. 1994. Pulsed growth of fungal hyphal tips. Proceedings of the National Academy of Sciences of the United States of America 91:12228-12232.

Løvtrup, S. 1983. Epigenetic mechanisms in the early amphibian embryo: cell differentiation and morphogenetic ele- 
ments. Biological Reviews 58:91-130. doi: 10.1111/j.1469185X. 1983.tb00381.x

Lutkenhaus, J., and Addinall, S. G. 1997. Bacterial cell division and the Z ring. Annual Review of Biochemistry 66:93-116. doi: 10.1146/annurev.biochem.66.1.93

Lymn, R. W., and Taylor, E.W. 1971. Mechanism of adenosine triphosphate hydrolysis by actomyosin. Biochemistry 10:4617-4623. doi: 10.1021/bi00801a004

Ma, H., Snook, L. A., Kaminskyj, S. G.W., and Dahms, T.E.S. 2005. Surface ultrastructure and elasticity in growing tips and mature regions of Aspergillus hyphae describe wall maturation. Microbiology 151:3679-3688. doi: 10.1099/mic.0.28328-0

Mabuchi, I. 1994. Cleavage furrow: timing of emergence of contractile ring actin filaments and establishment of the contractile ring by filament bundling in sea urchin eggs. Journal of Cell Science 107:1853-1862.

Mabuchi, I., Tsukita, Sh., Tsukita, Sa., and Sawai, T. 1988. Cleavage furrow isolated from newt eggs: Contraction, organization of the actin filaments, and protein components of the furrow. Proceedings of the National Academy of Sciences of the United States of America 85:5966-5970.

Marsh, L., and Letourneau, P. C. 1984. Growth activity of neurites without filopodial or lamellipodial in the presence of cytochalasin B. The Journal of Cell Biology 99:20412047. doi: 10.1083/jcb.99.6.2041

Marsland, D., and Landau, J. V. 1954. The mechanisms of cytokinesis: temperature-pressure studies on the cortical gel system in various marine eggs. Journal of Experimental Zoology 125:507-539. doi: 10.1002/jez.1401250307

Martin-Fernandez, M. L., Bordas, J., Diakun, G., Harries, J., Lowy, J., Mant, G. R., Svensson, A., and Towns-Andrews, E. 1994. Time-resolved X-ray diffraction studies of myosin head movements in live frog sartorius muscle during isometric and isotonic contractions. Journal of Muscle Research and Cell Motility 15:319-348. doi: 10.1007/ BF00123484

Mashkovtsev, A. A. 1935. Rol funktsii pri razvitii organov pozvonochnykh zhivotnykh [Role of function in the development of organs in vertebrates]. Trudy Laboratorii Evolyutsionnoi Morfologii AN SSSR, 2(3):31-52.

Mast, S. O. 1926. Structure, movement, locomotion, and stimulation in Amoeba. Journal of Morphology 41:347-425. doi: 10.1002/jmor.1050410205

Mast, S. O., and Hopkins, D. L. 1941. Regulation of the water content of Amoeba mira and adaptation to changes in the osmotic concentration of the surrounding medium. Journal of Cellular and Comparative Physiology 17:31-48. doi: 10.1002/jcp.1030170104

Masuda, T. 2003. An electromechanical model of myosin molecular motors. Journal of Theoretical Biology 225:507515. doi: 10.1016/S0022-5193(03)00302-3

Matsubara, I., and Elliott, G. F. 1972. X-ray diffraction studies on skinned single fibres of frog skeletal muscle. Journal of Molecular Biology 72:657-669. doi: 10.1016/00222836(72)90183-0

Matsudaira, P. T., and Burgess, D. R. 1979. Identification and organization of the components in the isolated microvillus cytoskeleton. The Journal of Cell Biology 83:667-673. doi: 10.1083/jcb.83.3.667

Maurel, C., and Chrispeels, M.J. 2001. Aquaporins. A molecular entry into plant water relations. Plant Physiology 125:135-138. doi: 10.1104/pp.125.1.135

Mazzochi, C., Benos, D.J., and Smith, P. R. 2006. Interaction of epithelial ion channels with the actin-based cytoskeleton. American Journal of Physiology - Renal Physiology 291:F1113-F1122.
Mehta, A. 2001. Myosin learns to walk. Journal of Cell Science 114:1981-1998.

Merkel, R., Simson, R., Simson, D. A., Hohenadl, M., Boulbitch, A., Wallraff, E., and Sackmann, E. 2000. A micromechanic study of cell polarity and plasma membrane cell body coupling in Dictyostelium. Biophysical Journal 79:707-719. doi: 10.1016/S0006-3495(00)76329-6

Messerli, M. A., Créton, R., Jaffe, L. F., and Robinson, K. R. 2000. Periodic increases in elongation rate precede increases in cytosolic $\mathrm{Ca}^{2+}$ during pollen tube growth. Developmental Biology 222:84-98. doi: 10.1006/dbio.2000.9709

Messerli, M. A., Danuser, G., and Robinson, K. R. 1999. Pulsatile influxes of $\mathrm{H}^{+}, \mathrm{K}^{+}$and $\mathrm{Ca}^{2+}$ lag growth pulses of Lilium longiflorum pollen tubes. Journal of Cell Science 112:1497-1509.

Messerli, M. A., and Robinson, K. R. 2003. Ionic and osmotic disruptions of the lily pollen tube oscillator: testing proposed models. Planta 217:147-157. doi: 10.1007/ s00425-003-0972-0

Miao, L., Vanderlinde, O., Stewart, M., and Roberts, T. M. 2003. Retraction in amoeboid cell motility powered by cytoskeletal dynamics. Science 302:1405-1407. doi: 10.1126/ science.1089129

Miller, D. D., Lancelle, S. A., and Hepler, P. K. 1996. Actin microfilaments do not form a dense meshwork in Lilium longiflorum pollen tube tips. Protoplasma 195:123-132. doi: 10.1007/BF01279191

Millman, B. M. 1998. The filament lattice of striated muscle. Physiological Reviews 78:359-391.

Millman, B. M., and Nickel, B. G. 1980. Electrostatic forces in muscle and cylindrical gel systems. Biophysical Journal 32:49-63. doi: 10.1016/S0006-3495(80)84915-0

Mills, J. W., and Mandel, L. J. 1994. Cytoskeletal regulation of membrane transport events. The FASEB Journal 8:1161-1165.

Mills, J.W, Pedersen, S. F, Walmod, P.S, and Hoffmann, E. K. 2000. Effect of cytochalasins on F-actin and morphology of Ehrlich ascites tumor cells. Experimental Cell Research 261:209-219. doi: 10.1006/excr.2000.5032

Miranda, A. F., Godman, G. C., Deitch, A. D., and Tanenbaum, S.W.1974. Action of cytochalasin D on cells of established lines. I. Early events. The Journal of Cell Biology 61:481-500. doi: 10.1083/jcb.61.2.481

Mitchison, T., and Kirschner, M. 1988. Cytoskeletal dynamics and nerve growth. Neuron 1:761-772. doi: 10.1016/08966273(88)90124-9

Mogilner, A. 2006. On the edge: modeling protrusion. Current Opinion in Cell Biology 18:32-39. doi: 10.1016/j. ceb.2005.11.001

Mogilner, A., and Oster, G. 1996. Cell motility driven by actin polymerization. Biophysical Journal 71:3030-3045. doi: 10.1016/S0006-3495(96)79496-1

Money, N.P. 2001. The pulse of the machine - reevaluating tip-growth methodology. New Phytologist 151:553-555. doi: 10.1046/j.0028-646x.2001.00243.x

Money, N.P., and Harold, F. M. 1993. Two water molds can grow without measurable turgor pressure. Planta 190:426-430. doi: 10.1007/BF00196972

Moore, A. R., and Burt, A.S. 1939. On the locus and nature of the forces causing gastrulation in the embryos of Dendraster excentricus. Journal of Experimental Zoology 82:159-171. doi: 10.1002/jez.1400820107

Moore, K. A., Polte, T., Huang, S., Shi, B., Alsberg, E., Sunday, M. E., and Ingber, D. E. 2005. Control of basement membrane remodeling and epithelial branching morphogenesis in embryonic lung by Rho and cytoskeletal tension. Developmental Dynamics 232:268-281. doi: 10.1002/ dvdy.20237 
Mooseker, M. S., Pollard, T. D., and Wharton, K. A. 1982. Nucleated polymerization of actin from the membraneassociated ends of microvillar filaments in the intestinal brush border. The Journal of Cell Biology 95:223-233. doi: 10.1083/jcb.95.1.223

Mooseker, M. S., and Tilney, L. G. 1975. Organization of an actin filament-membrane complex. The Journal of Cell Biology 67:725-743. doi: 10.1083/jcb.67.3.725

Moreno N., Colaço R., Feijó J. A. 2007. The pollen tube oscillator: integrating biophysics and biochemistry into cellular growth and morphogenesis; pp. 39-62 in Mancuso, S., and Shabala, S. (eds.), Rhythms in plants: phenomenology, mechanisms, and adaptive significance. SpringerVerlag, Berlin, Heidelberg.

Morris, C. E., Lesiuk, H., and Mills, L. R. 1997. How do neurons monitor their mechanical status? The Biological Bulletin 192:118-120. doi: 10.2307/1542582

Morton, W. M., Ayscough, K. R., and McLaughlin, P. J. 2000. Latrunculin alters the actin-monomer subunit interface to prevent polymerization. Nature Cell Biology 2:376-378. doi: $10.1038 / 35014075$

Müller, M. J. I., Klumpp, S., and Lipowsky, R. 2008. Tug-of-war as a cooperative mechanism for bidirectional cargo transport by molecular motors. Proceedings of the $\mathrm{Na}$ tional Academy of Sciences of the United States of America 105:4609-4614. doi: 10.1073/pnas.0706825105

Mullins, J.T., and Ellis, E. A. 1974. Sexual morphogenesis in Achlya: ultrastructural basis for the hormonal induction of antheridial hyphae. Proceedings of the National Academy of Sciences of the United States of America 71:13471350. doi: 10.1073/pnas.0706825105

Munevar, S., Wang, Y.-L., and Dembo, M. 2001. Traction force microscopy of migrating normal and H-ras transformed 3T3 fibroblasts. Biophysical Journal 80:1744-1757. doi: 10.1016/S0006-3495(01)76145-0

Murphy, R. A. 1994. What is special about smooth muscle? The significance of covalent crossbridge regulation. The FASEB Journal 8:311-318.

Murphy, R.A., Herlihy, J.T., and Megerman, J. 1974. Forcegenerating capacity and contractile protein content of arterial smooth muscle. The Journal of General Physiology 64:691-705. doi: 10.1085/jgp.64.6.691

Nadol, J. B., Gibbins, J. R., and Porter, K. R. 1969. A reinterpretation of the structure and development of the basement lamella: an ordered array of collagen in fish skin. Developmental Biology 20:304-331. doi: 10.1016/00121606(69)90017-7

Nakajima, Y., and Burke, R. D. 1996. The initial phase of gastrulation in sea urchins is accompanied by the formation of bottle cells. Developmental Biology 179:436-446. doi: 10.1006/dbio.1996.0273

Nakanishi, Y., Nogawa, H., Hashimoto, Y., Kishi, J.-I., and Hayakawa, T. 1988. Accumulation of collagen III at the cleft points of developing mouse submandibular epithelium. Development 104:51-59.

Nakanishi, Y., Sugiura, F., Kishi, J.-I., and Hayakawa, T. 1986. Scanning electron microscopic observation of mouse embryonic submandibular glands during initial branching: preferential localization of fibrillar structures at the mesenchymal ridges participating in cleft formation. Journal of Embryology and Experimental Morphology 96:65-77.

Naylor, G. R. S., Bartels, E. M., Bridgman, T. D., and Elliott, G. F. 1985. Donnan potentials in rabbit psoas muscle in rigor. Biophysical Journal 48:47-59. doi: 10.1016/S00063495(85)83759-0

Nelson, G. A., Roberts, T. M., and Ward, S. 1982. Caenorhabditis elegans spermatozoan locomotion: amoeboid move- ment with almost no actin. The Journal of Cell Biology 92:121-131. doi: 10.1083/jcb.92.1.121

Neujahr, R., Heizer, C., and Gerisch, G. 1997. Myosin Il-independent processes in mitotic cells of Dictyostelium discoideum: redistribution of the nuclei, re-arrangement of the actin system and formation of the cleavage furrow. Journal of Cell Science 110:123-137.

Newman, S. A., and Frisch, H. L. 1979. Dynamics of skeletal pattern formation in developing chick limb. Science 205:662-668. doi: 10.1126/science.462174

Niiro, N., and Ikebe, M. 2001. Zipper-interacting protein kinase induces $\mathrm{Ca}^{2+}$-free smooth muscle contraction via myosin light chain phosphorylation. Journal of Biological Chemistry 276:29567-29574. doi: 10.1074/jbc.M102753200

Odell, G. M., Oster, G., Alberch, P., and Burnside, B. 1981. The mechanical basis of morphogenesis. I. Epithelial folding and invagination. Developmental Biology 85:446-462. doi: 10.1016/0012-1606(81)90276-1

Offer, G. 2006. Fifty years on: where have we reached? Journal of Muscle Research and Cell Motility 27:205-213. doi: 10.1007/s10974-006-9062-9

O’Neill, W. C. 1999. Physiological significance of volume-regulatory transporters. American Journal of Physiology - Cell Physiology 276:C995-C1011.

Oparka, K. J., Prior, D. A. M., and Harris, N. 1990. Osmotic induction of fluid-phase endocytosis in onion epidermal cells. Planta 180:555-561. doi: 10.1007/BF02411454

Oster, G. F., and Perelson, A. S. 1987. The physics of cell motility. Journal of Cell Science Suppl. 8:35-54. doi: 10.1242/ jcs.1987.Supplement_8.3

Ovečka, M., Lang, I., Baluška, F., Ismail, A., Illeš, P., and Lichtscheidl, I. K. 2005. Endocytosis and vesicle trafficking during tip growth of root hairs. Protoplasma 226:39-54. doi: 10.1007/s00709-005-0103-9

Paller, M. S. 1994. Lateral mobility of Na,K-ATPase and membrane lipids in renal cells. Importance of cytoskeletal integrity. The Journal of Membrane Biology 142:127-135. doi: 10.1007/BF00233390

Paluch, E., Sykes, C., Prost, J., and Bornens, M. 2006. Dynamic modes of the cortical actomyosin gel during cell locomotion and division. Trends in Cell Biology 16:5-10. doi: 10.1016/j.tcb.2005.11.003

Papadopoulos, M. C., Saadoun, S., and Verkman, A. S. 2008. Aquaporins and cell migration. Pflügers Archiv - European Journal of Physiology 456:693-700. doi: 10.1007/s00424007-0357-5

Parnass, Z., Tashiro, A., and Yuste, R. 2000. Analysis of spine morphological plasticity in developing hippocampal pyramidal neurons. Hippocampus 10:561-568. doi: 10.1002/1098-1063(2000)10:5<561::AID-HIPO6>3.0.CO;2-X

Parre, E., and Geitmann, A. 2005. Pectin and the role of the physical properties of the cell wall in pollen tube growth of Solanum chacoense. Planta 220:582-592. doi: 10.1007/ s00425-004-1368-5

Patan, S. 2000. Vasculogenesis and angiogenesis as mechanisms of vascular network formation, growth and remodeling. Journal of Neuro-Oncology 50:1-15. doi: 10.1023/A:1006493130855

Pedersen, S. F., Hoffmann, E. K., and Mills, J. W. 2001. The cytoskeleton and cell volume regulation. Comparative Biochemistry and Physiology - Part A: Molecular and Integrative Physiology 130:385-399.

Pepicelli, C. V., Lewis, P. M., and McMahon, A. P. 1998. Sonic hedgehog regulates branching morphogenesis in the mammalian lung. Current Biology 8:1083-1086. doi: 10.1016/S0960-9822(98)70446-4

Peterson, J. A., Tian, B., Geiger, B., and Kaufman, P. L. 2000. Effect of latrunculin-B on outflow facility in monkeys. 
Experimental Eye Research 70:307-313. doi: 10.1006/ exer.1999.0797

Petrunkina, A. M., Hebel, M., Waberski, D., Weitze, K. F., and Töpfer-Petersen, E. 2004. Requirement for an intact cytoskeleton for volume regulation in boar spermatozoa. Reproduction 127:105-115. doi: 10.1530/rep.1.00047

Pfenninger, K. H., and Johnson, M.P. 1983. Membrane biogenesis in the sprouting neuron. I. Selective transfer of newly synthesized phospholipid into the growing neurite. The Journal of Cell Biology 97:1038-1042. doi: 10.1083/jcb.97.4.1038

Pierson, E. S., Miller, D. A., Callaham, D. A., Shipley, A. M., Rivers, B. A., Cresti, M., and Hepler, P. K. 1994. Pollen tube growth is coupled to the extracellular calcium ion flux and the intracellular calcium gradient: effect of BAPTAtype buffers and hypertonic medium. The Plant Cell 6:1815-1828. doi: 10.1105/tpc.6.12.1815

Pierson, E. S., Miller, D. D., Callaham, D. A., van Aken, J., Hackett, G., and Hepler, P. K. 1996. Tip-localized calcium entry fluctuates during pollen tube growth. Developmental Biology 174:160-173. doi: 10.1006/dbio.1996.0060

Polevoy, V.V., and Salamatova, T.S.1985. Evolyutsiya sposobov dvizheniya u rastenii [Evolution of movement in plants]; pp. 188-200 in Polevoy, V. V., Maslova, Yu. I. (eds.), Evolution of functions in the plant world. Izdatelstvo Leningradskogo gosudarstvennogo universiteta, Leningrad.

Pollard, T.D. 2000. Reflections on a quarter century of research on contractile systems. Trends in Biochemical Sciences 25:607-611. doi: 10.1016/S0968-0004(00)01719-9

Pollard, T. D. 2003. The cytoskeleton, cellular motility and the reductionist agenda. Nature 422:741-745. doi: 10.1038/ nature01598

Pollard, T. D., and Borisy, G. G. 2003. Cellular motility driven by assembly and disassembly of actin filaments. Cell 112:453-465. doi: 10.1016/S0092-8674(03)00120-X

Ponti, A., Machacek, M., Gupton, S. L., Waterman-Storer, C. M., and Danuser, G. 2004. Two distinct actin networks drive the protrusion of migrating cells. Science 305:1782-1786. doi: $10.1126 /$ science.1100533

Pypaert, M., Mundy, D., Souter, E., Labbé, J.-C., and Warren, G. 1991. Mitotic cytosol inhibits invagination of coated pits in broken mitotic cells. The Journal of Cell Biology 114:1159-1166. doi: 10.1083/jcb.114.6.1159

Qualmann, B., and Mellor, H. 2003. Regulation of endocytic traffic by Rho GTPases. Biochemical Journal 371:233-241. doi: 10.1042/bj20030139

Radice, G. P. 1980. Locomotion and cell-substratum contacts of Xenopus epidermal cells in vitro and in situ. Journal of Cell Science 44:201-223.

Raucher, D., and Sheetz, M. P. 1999. Membrane expansion increases endocytosis rate during mitosis. The Journal of Cell Biology 144:497-506. doi: 10.1083/jcb.144.3.497

Rayment, I., Holden, H. M., Whittaker, M., Yohn, C. B., Lorenz, M., Holmes, K. C., and Milligan, R. A. 1993a. Structure of the actin-myosin complex and its implications for muscle contraction. Science 261:58-65.

Rayment, I., Rypnieswski, W. R., Schmidt-Bäse, K., Smith, R., Tomchick, D. R., Benning, M. M., Winkelmann, D. A., Wesenberg, G., and Holden, H. M. 1993b. Three-dimensional structure of myosin subfragment-1: a molecular motor. Science 261:50-58.

Raynaud, A., Adrian, M., and Kouprach, S. 1974. Etude, au microscope electronique, des ebauches des membres de l'orvet (Anguis fragilis L.) et du lezard vert (Lacerta viridis Laur.). Annales d'Embryologie et de Morphogenèse 7:243263.

Reese, E. L., and Haimo, L. T. 2000. Dynein, dynactin, and kinesin II's interaction with microtubules is regulated during bidirectional organelle transport. The Journal of Cell Biology 151:155-166. doi: 10.1083/jcb.151.1.155

Regini, J. W., and Elliott, G. F. 2001. The effect of temperature on the Donnan potentials in biological polyelectrolyte gels: cornea and striated muscle. International Journal of Biological Macromolecules 28:245-254. doi: 10.1016/ S0141-8130(01)00120-9

Ridley, A. J., Schwartz, M. A., Burridge, K., Firtel, R. A., Ginsberg, M. H., Borisy, G., Parsons, J.T., and Horwitz, A. R. 2003. Cell migration: integrating signals from front to back. Science 302:1704-1709. doi: 10.1126/science.1092053

Roberts, T.M., and Stewart, M. 2000. Acting like actin: the dynamics of the nematode major sperm protein (MSP) cytoskeleton indicate a push-pull mechanism for amoeboid cell motility. The Journal of Cell Biology 149:7-12. doi: 10.1083/jcb.149.1.7

Roberts, T.M., and Streitmatter, G. 1984. Membrane-substrate contact under the spermatozoon of Caenorhabditis elegans, a crawling cell that lacks filamentous actin. Journal of Cell Science 69:117-126.

Rogers, S. S., Waigh, T. A., and Lu, J. R. 2008. Intracellular microrheology of motile Amoeba proteus. Biophysical Journal 94:3313-3322. doi: 10.1529/biophysj.107.123851

Rhumbler, L. 1902. Zur Mechanik des Gastrulationsvorganges insbesondere der Invagination. Archiv für Entwickelungsmechanik 14:401-476.

Saadoun, S., Papadopoulos, M.C., Watanabe, H., Yan, D., Manley, G. T., and Verkman, A. S. 2005. Involvement of aquaporin-4 in astroglial cell migration and glial scar formation. Journal of Cell Science 118:5691-5698. doi: $10.1242 / j c s .02680$

Saida, K., and Nonomura, Y. 1978. Characteristics of $\mathrm{Ca}^{2+}$ and $\mathrm{Mg}^{2+}$-induced tension development in chemically skinned smooth muscle fibers. The Journal of General Physiology 72:1-14. doi: 10.1085/jgp.72.1.1

Salmon, E. D., and Wolniak, S. M. 1990. Role of microtubules in stimulating cytokinesis in animal cells. Annals of the New York Academy of Sciences 582:88-98. doi: 10.1111/ j.1749-6632.1990.tb21670.x

Salpeter, M. M., and Singer, M.1960. Differentiation of the submicroscopic adepidermal membrane during limb regeneration in adult Triturus, including a note on the use of the term basement membrane. The Anatomical Record 136:27-40. doi: 10.1002/ar.1091360104

Sanger, J. M, and Sanger, J. W. 1980. Banding and polarity of actin filaments in interphase and cleaving cells. The Journal of Cell Biology 86:568-575. doi: 10.1083/jcb.86.2.568

Sanger, J. M., and Sanger, J.W. 2000. Assembly of cytoskeletal proteins into cleavage furrows of tissue culture cells. Microscopy Research and Technique 49:190-201. doi: 10.1002/(SICI)1097-0029(20000415)49:2<190::AIDJEMT12>3.0.CO;2-B

Sanger, J. W., and Holtzer, H. 1972. Cytochalasin B: Effects on cell morphology, cell adhesion, and mucopolysaccharide synthesis. Proceedings of the National Academy of Sciences of the United States of America 69:253-257.

Sanger, J. W., Sanger, J. M., and Jockusch, B. M. 1983. Differences in the stress fibers between fibroblasts and epithelial cells. The Journal of Cell Biology 96:961-969. doi: 10.1083/jcb.96.4.961

Satoh, H., Ueda, T., and Kobatake, Y. 1985. Oscillations in cell shape and size during locomotion and in contractile activities of Physarum polycephalum, Dictyostelium discoideum, Amoeba proteus and macrophages. Experimental Cell Research 156:79-90. doi: 10.1016/0014-4827(85)90263-0

Saunders, P. T., and Trinci, A. P.J. 1979. Determination of tip shape in fungal hyphae. Journal of General Microbiology 110:469-473. 
Savion, N., and Selinger, Z. 1978. Morphological changes in rat pancreatic slices associated with inhibition of enzyme secretion by high concentrations of secretagogues. The Journal of Cell Biology 76:467-482. doi: 10.1083/ jcb.76.2.467

Schatten, G., Schatten, H., Spector, I., Cline, C., Paweletz, N., Simerly, C., and Petzelt, C. 1986. Latrunculin inhibits the microfilament-mediated processes during fertilization, cleavage and early development in sea urchins and mice. Experimental Cell Research 166:191-208. doi: 10.1016/0014-4827(86)90519-7

Schliwa, M., and Woehlke, G. 2003. Molecular motors. Nature 422:759-765. doi: 10.1038/nature01601

Schmalhausen, O.I.1975. Osyotr Acipenser güldenstädti colchicus [Sturgeon Acipenser güldenstädti colchicus]; pp. 264-277 in Dettlaff, T. A. (ed.), Objects of Developmental Biology. Izdatelstvo Nauka, Moscow.

Schnapp, B.J., and Reese, T.S. 1982. Cytoplasmic structure in rapid-frozen axons. The Journal of Cell Biology 94:667679. doi: 10.1083/jcb.94.3.667

Schneider, S. W., Pagel, P., Rotsch, C., Danker, T., Oberleithner, H., Radmacher, M., and Schwab, A. 2000. Volume dynamics in migrating epithelial cells measured with atomic force microscopy. Pflügers Archiv - European Journal of Physiology 439:297-303.

Schoenwolf, G.C. 1979. Histological and ultrastructural observations of tail bud formation in the chick embryo. The Anatomical Record 193:131-148. doi: 10.1002/ ar.1091930108

Schoenwolf, G. C., and Smith, J. L. 1990. Mechanisms of neurulation: traditional viewpoint and recent advances. Development 109:243-270.

Schroeder, T.E.1972. The contractile ring. II. Determining its brief existence, volumetric changes, and vital role in cleaving Arbacia eggs. The Journal of Cell Biology 53:419434. doi: 10.1083/jcb.53.2.419

Schroeder, T. E. 1973. Cell constriction: contractile role of microfilaments in division and development. American Zoologist 13:949-960. doi: 10.1093/icb/13.4.949

Schroeder, T.E. 1990. The contractile ring and furrowing in dividing cells. Annals of the New York Academy of Sciences 582:78-87. doi: 10.1111/j.1749-6632.1990.tb21669.x

Schwab, A. 2001. Function and spatial distribution of ion channels and transporters in cell migration. American Journal of Physiology - Renal Physiology 280:739-747.

Schwab, A., Nechyporuk-Zloy, V., Fabian, A., and Stock, C. 2007. Cells move when ions and water flow. Pflügers Archiv - European Journal of Physiology 453:F421-F432.

Schwiebert, E. M., Mills, J. W., and Stanton, B. A. 1994. Actinbased cytoskeleton regulates a chloride channel and cell volume in a renal cortical collecting duct cell line. Journal of Biological Chemistry 269:7081-7089.

Seow, C. Y., Pratusevich, V. R., and Ford, L. E. 2000. Series-toparallel transition in the filament lattice of airway smooth muscle. Journal of Applied Physiology 89:869-876.

Sepsenwol, S., Ris, H., and Roberts, T. M. 1989. A unique cytoskeleton associated with crawling in the amoeboid sperm of the nematode, Ascaris suum. The Journal of Cell Biology 108:55-66. doi: 10.1083/jcb.108.1.55

Sheetz, M.P. 2001. Cell control by membrane-cytoskeleton adhesion. Nature Reviews Molecular Cell Biology 2:392396. doi: 10.1038/35073095

Shi, X., Gillespie, P. G., and Nuttall, A. L. 2005. Na+ influx triggers bleb formation on inner hair cells. American Journal of Physiology - Cell Physiology 288:C1332-C1341.

Shimizu, T. 1990. Polar body formation in Tubifex eggs. Annals of the New York Academy of Sciences 582:260-272. doi: 10.1111/j.1749-6632.1990.tb21685.x
Siegman, M.J., Butler, T.M., Mooers, S. U., and Davies, R.E.1980. Chemical energetics of force development, force maintenance, and relaxation in mammalian smooth muscle. The Journal of General Physiology 76:609-629. doi: 10.1085/jgp.76.5.609

Singley, C. T., and Solursh, M. 1981. The spatial distribution of hyaluronic acid and mesenchymal condensation in the embryonic chick wing. Developmental Biology 84:102120. doi: 10.1016/0012-1606(81)90375-4

Small, J. V. 1974. Contractile units in vertebrate smooth muscle cells. Nature 249:324-327. doi: 10.1038/249324a0

Small, J.V., Herzog, M., and Anderson, K. 1995. Actin filament organization in the fish keratocyte lamellipodium. The Journal of Cell Biology 129:1275-1286. doi: 10.1083/ jcb.129.5.1275

Small, J. V., Herzog, M., Barth, M., and Draeger, A. 1990. Supercontracted state of vertebrate smooth muscle cell fragments reveals myofilament lengths. The Journal of Cell Biology 111:2451-2461. doi: 10.1083/jcb.111.6.2451

Small, J. V., and Resch, G. P. 2005. The comings and goings of actin: coupling protrusion and retraction in cell motility. Current Opinion in Cell Biology 17:517-523. doi: 10.1016/j. ceb.2005.08.004

Small, J. V., Stradal, T., Vignal, E., and Rottner, K. 2002. The lamellipodium: where motility begins. Trends in Cell Biology 12:112-120. doi: 10.1016/S0962-8924(01)02237-1

Smith, A. A., Searls, R. L., and Hilfer, S. R. 1975. Differential accumulation of extracellular materials beneath the ectoderm during development of the embryonic chick limb and flank regions. Developmental Biology 46:222-226. doi: 10.1016/0012-1606(75)90100-1

Somlyo, A. P., and Somlyo, A. V. 1994. Signal transduction and regulation in smooth muscle. Nature 372:231-236. doi: 10.1038/372231a0

Sorokin, S. 1961. A study of development in organ cultures of mammalian lungs. Developmental Biology 3:60-83. doi: 10.1016/0012-1606(61)90010-0

Spector, I., Schochet, N.R., Blasberger, D., and Kashman, Y. 1989. Latrunculins - novel marine macrolides that disrupt microfilament organization and affect cell growth: I. Comparison with cytochalasin D. Cell Motility and the Cytoskeleton 13:127-144. doi: 10.1002/cm.970130302

Spooner, B. S. 1973. Microfilaments, cell shape changes, and morphogenesis of salivary epithelium. American Zoologist 13:1007-1022. doi: 10.1093/icb/13.4.1007

Steinberg, G. 2007. Hyphal growth: a tale of motors, lipids, and the spitzenkörper. Eukaryotic Cell 6:351-360. doi: 10.1128/EC. 00381-06

Stockem, W., and Kłopocka, W. 1988. Ameboid movement and related phenomena. International Review of Cytology 112:137-183.

Stossel, T.P. 1993. On the crawling of animal cells. Science 260:1086-1094. doi: 10.1126/science.8493552

Stroeva, O.G.1950. Etapy formirovaniya plavnikov lichinok amfibii [Stages of formation of fins in amphibian larvae]. Doklady Akademii Nauk SSSR 70:145-147.

Strohmeier, R., and Bereiter-Hahn, J. 1987. Hydrostatic pressure in epidermal cells is dependent on Ca-mediated contractions. Journal of Cell Science 88:631-640.

Suzuki, M., Miyazaki, K., Ikeda, M., Kawaguchi, Y., and Sakai, O. 1993. F-actin network may regulate a $\mathrm{Cl}^{-}$channel in renal proximal tubule cells. The Journal of Membrane Biology 134:31-39. doi: 10.1007/BF00233473

Svitkina, T. M., Verkhovsky, A. B., McQuade, K. M., and Borisy, G. G. 1997. Analysis of the actin-myosin II system in fish epidermal keratocytes: mechanism of cell body translocation. The Journal of Cell Biology 139:397-415. doi: 10.1083/jcb.139.2.397 
Sytina, L. A., Medvedeva, I. M., and Godina, L. B. 1987. Razvitie sibirskogo uglozuba [Development of Siberian Salamander]. 86 pp. Izdatelstvo Nauka, Moscow.

Taheri-Talesh, N., Horio, T., Araujo-Bazán, L., Dou, X., Espeso, E. A., Peñalva, M. A., Osmani, S. A., and Oakley, B. R. 2008. The tip growth apparatus of Aspergillus nidulans. Molecular Biology of the Cell 19:1439-1449. doi: 10.1091/mbc. E07-05-0464

Takata, H., and Kominami, T. 2001. Shrinkage and expansion of blastocoel affect the degree of invagination in sea urchin embryos. Zoological Science 18:1097-1105. doi: 10.2108/zsj.18.1097

Taylor, A.R., Manison, N. F. H., Fernandez, C., Wood, J., and Brownlee, C. 1996. Spatial organization of calcium signaling involved in cell volume control in the Fucus rhizoid. The Plant Cell 8:2015-2031. doi: 10.1105/tpc.8.11.2015

Taylor, L. P., and Hepler, P. K. 1997. Pollen germination and tube growth. Annual Review of Plant Physiology and Plant Molecular Biology 48:461-491. doi: 10.1146/annurev.arplant.48.1.461

Telley, I. A., and Denoth, J. 2007. Sarcomere dynamics during muscular contraction and their implications to muscle function. Journal of Muscle Research and Cell Motility 28:89-104. doi: 10.1007/s10974-007-9107-8

Tesi, C., Colomo, F., Piroddi, N., and Poggesi, C. 2002. Characterization of the cross-bridge force-generating step using inorganic phosphate and BDM in myofibrils from rabbit skeletal muscles. The Journal of Physiology 541:187-199. doi: 10.1113/jphysiol.2001.013418

Tickle, C., and Trinkaus, J. P. 1977. Some clues as to the formation of protrusions by Fundulus deep cells. Journal of Cell Science 26:139-150.

Tilney, L. G., and Inoué, S. 1982. Acrosomal reaction of Thyone sperm . II. The kinetics and possible mechanism of acrosomal process elongation. The Journal of Cell Biology 93:820-827. doi: 10.1083/jcb.93.3.820

Tilney, L. G., and Inoué, S. 1985. Acrosomal reaction of the Thyone sperm. III. The relationship between actin assembly and water influx during the extension of the acrosomal process. The Journal of Cell Biology 100:1273-1283. doi: 10.1083/jcb.100.4.1273

Tilney, L. G., and Jaffe, L. A. 1981. Actin, microvilli, and the fertilization cone of sea urchin eggs. The Journal of Cell Biology 87:771-782. doi: 10.1083/jcb.87.3.771

Tilney, L. G., and Portnoy, D. A. 1989. Actin filaments and the growth, movement, and spread of the intracellular bacterial parasite, Listeria monocytogenes. The Journal of Cell Biology 109:1597-1608. doi: 10.1083/jcb.109.4.1597

Torgerson, R. R., and McNiven, M.A. 1998. The actinmyosin cytoskeleton mediates reversible agonistinduced membrane blebbing. Journal of Cell Science 111:2911-2922.

Tosney, K.W., and Wessells, N. K.1983. Neuronal motility: The ultrastructure of veils and microspikes correlates with their motile activities. Journal of Cell Science 61: 389-411.

Toyoshima, Y.Y., Kron, S.J., McNally, E. M., Niebling, K. R., Toyoshima, C., and Spudich, J. A. 1987. Myosin subfragment-1 is sufficient to move actin filaments in vitro. $\mathrm{Na}$ ture 328:536-539. doi: 10.1038/328536a0

Trimmer, W.S. N. 1989. Microrobots and micromechanical systems. Sensors and Actuators 19:267-287. doi: 10.1016/0250-6874(89)87079-9

Trinkaus J.P. 1969. Cells into organs. The forces that shape the embryo. 238 pp. Prentice-Hall Inc., New Jersey.

Tsakiridis, T., Vranic, M., and Klip, A. 1994. Disassembly of the actin network inhibits insulin-dependent stimulation of glucose transport and prevents recruitment of glucose transporters to the plasma membrane. Journal of Biological Chemistry 269:29934-29942.

Tyreman, M.J.A., and Molloy, J. E. 2003. Molecular motors: nature's nanomachines. IEE Proceedings - Nanobiotechnology 150:95-102. doi: 10.1049/ip-nbt:20031172

Umemoto, S., and Sellers, J. R. 1990. Characterization of in vitro motility assays using smooth muscle and cytoplasmic myosins. Journal of Biological Chemistry 265:14864-14869.

Uyeda, T. Q. P., and Nagasaki, A. 2004. Variations on a theme: the many modes of cytokinesis. Current Opinion in Cell Biology 16:1-6. doi: 10.1016/j.ceb.2003.11.004

Vale, R.D.1999. Millennial musings on molecular motors. Trends in Cell Biology 9:38-42. doi: 10.1016/S09680004(99)01476-0

VanBuren, P., Work, S. S., and Warshaw, D. M. 1994. Enhanced force generation by smooth muscle myosin in vitro. Proceedings of the National Academy of Sciences of the United States of America 91:202-205.

Verkhovsky, A. B., Svitkina, T. M., and Borisy, G. G. 1995. Myosin II filament assemblies in the active lamella of fibroblasts: their morphogenesis and role in the formation of actin filament bundles. The Journal of Cell Biology 131:989-1002. doi: 10.1083/jcb.131.4.989

Verkman, A. S. 2005. More than just water channels: unexpected cellular roles of aquaporins. Journal of Cell Science 118:3225-3232. doi: 10.1242/jcs.02519

Verkman, A. S., Binder, D. K., Bloch, O., Auguste, K., and Papadopoulos, M. C. 2006. Three distinct roles of aquaporin-4 in brain function revealed by knockout mice. Biochimica et Biophysica Acta 1758:1085-1093. doi: 10.1016/j. bbamem.2006.02.018

Vidali, L., and Hepler, P. K. 2001. Actin and pollen tube growth. Protoplasma 215:64-76. doi: 10.1007/BF01280304

Wang, Q., Bilan, P.J., Tsakiridis, T., Hinek, A., and Klip, A. 1998. Actin filaments participate in the relocalization of phosphatidylinositol 3-kinase to glucose transporter-containing compartments and in the stimulation of glucose uptake in 3T3-L1 adipocytes. Biochemical Journal 331:917928. doi: 10.1042/bj3310917

Warshaw, D. M., McBride, W. J., and Work, S. S. 1987. Corkscrew-like shortening in single smooth muscle cells. Science 236:1457-1459. doi: 10.1126/science.3109034

Warshaw, D. M., Szarek, J. L, Hubbard, M.S., and Evans, J. N. 1986. Pharmacology and force development of single freshly isolated bovine carotid artery smooth muscle cells. Circulation Research 58:399-406. doi: 10.1161/01. RES. 58.3.399

Wehland, J., Weber, K., Gawlitta, W., and Stockem, W. 1979. Effects of the actin-binding protein DNAase I on cytoplasmic streaming and ultrastructure of Amoeba proteus. An attempt to explain amoeboid movement. Cell and Tissue Research 199:353-372. doi: 10.1007/BF00236075

Wehner, F., Olsen, H., Tinel, H., Kinne-Saffran, E., and Kinne, R.K.H. 2003. Cell volume regulation: osmolytes, osmolyte transport, and signal transduction. Reviews of Physiology, Biochemistry and Pharmacology 148:1-80. doi: 10.1007/s10254-003-0009-x

Wessels, J.G.H.1993. Wall growth, protein excretion and morphogenesis in fungi. New Phytologist 123:397-413. doi: 10.1111/j.1469-8137.1993.tb03751.x

Wessells, N. K. 1970. Mammalian lung development: interactions in formation and morphogenesis of tracheal buds. Journal of Experimental Zoology 175:455-466. doi: 10.1002/jez.1401750405

Williams, J. M, and Daniel, C. W. 1983. Mammary ductal elongation: differentiation of myoepithelium and basal lamina during branching morphogenesis. Developmental Biology 97:274-290. doi: 10.1016/0012-1606(83)90086-6 
Wilson, D. P., Sutherland, C., and Walsh, M. P. 2002. Ca ${ }^{2+}$ activation of smooth muscle contraction. Evidence for the involvement of calmodulin that is bound to the tritoninsoluble fraction even in the absence of $\mathrm{Ca}^{2+}$. Journal of Biological Chemistry 277:2186-2192. doi: 10.1074/jbc. M110056200

Wittmann, T., and Waterman-Storer, C. M. 2001. Cell motility: can Rho GTPases and microtubules point the way? Journal of Cell Science 114:3795-3803.

Wordeman, L., and Cande, W. Z. 1990. Cytokinesis by furrowing in diatoms. Annals of the New York Academy of Sciences 582:252-259. doi: 10.1111/j.1749-6632.1990. tb21684.x

Xu, J.-Q., Harder, B. A., Uman, P., and Craig, R. 1996. Myosin filament structure in vertebrate smooth muscle. The Journal of Cell Biology 134:53-66. doi: 10.1083/jcb.134.1.53

Yaffe, M. P. 1999. The machinery of mitochondrial inheritance and behavior. Science 283:1493-1497. doi: 10.1126/science.283.5407.1493

Yamada, K. M., Spooner, B. S., and Wessells, N. K. 1970. Axon growth: roles of microfilaments and microtubules. Proceedings of the National Academy of Sciences of the United States of America 66:1206-1212.

Yamada, K. M., Spooner, B. S, and Wessells, N. K. 1971. Ultrastructure and function of growth cones and axons of cultured nerve cells. The Journal of Cell Biology 49:614-635. doi: 10.1083/jcb.49.3.614

Yamazaki, D., Fujiwara, T., Suetsugu, S., and Takenawa, T.2005. A novel function of WAVE in lamellipodia: WAVE1 is required for stabilization of lamellipodial protrusions during cell spreading. Genes to Cells 10:381-392. doi: 10.1111/j.1365-2443.2005.00845.x

Yanagida, T., Kitamura, K., Tanaka, H., Iwane, A. H., and Esaki, S. 2000. Single molecule analysis of the actomyosin motor. Current Opinion in Cell Biology 12:20-25.

Yarar, D., Waterman-Storer, C. M., and Schmid, S. L. 2005. A dynamic actin cytoskeleton functions at multiple stages of clathrin-mediated endocytosis. Molecular Biology of the Cell 16:964-975. doi: 10.1091/mbc.E04-09-0774

Ybot-Gonzalez, P., and Copp, A.J.1999. Bending of the neural plate during mouse spinal neurulation is independent of actin microfilaments. Developmen- tal Dynamics 215:273-283. doi: 10.1002/(SICI)10970177(199907)215:3<273::AID-AJA9>3.0.CO;2-H

Yoshida, K., and Inouye, K. 2001. Myosin II-dependent cylindrical protrusions induced by quinine in Dictyostelium: antagonizing effects of actin polymerization at the leading edge. Journal of Cell Science 114:2155-2165.

Yoshida, K., and Soldati, T.2006. Dissection of amoeboid movement into two mechanically distinct modes. Journal of Cell Science 119:3833-3844. doi: 10.1242/jcs.03152

Yumura, S. 2001. Myosin II dynamics and cortical flow during contractile ring formation in Dictyostelium cells. The Journal of Cell Biology 154:137-145. doi: 10.1083/ jcb.200011013

Yuri, K., Wakayama, J., and Yamada, T. 1998. Isometric contractile properties of single myofibrils of rabbit skeletal muscle. The Journal of Biochemistry 124:565-571. doi: 10.1093/oxfordjournals.jbchem.a022149

Zang, J.-H., Cavet, G., Sabry, J.H., Wagner, P., Moores, S. L., and Spudich, J. A. 1997. On the role of myosin-II in cytokinesis: division of Dictyostelium cells under adhesive and nonadhesive conditions. Molecular Biology of the Cell 8:2617-2629. doi: 10.1091/mbc.8.12.2617

Zicha, D., Dobbie, I. M., Holt, M. R., Monypenny, J., Soong, D. Y. H., Gray, C., and Dunn, G. A. 2003. Rapid actin transport during cell protrusion. Science 300:142-145. doi: 10.1126/science.1082026

Zonia, L., Müller, M., and Munnik, T. 2006. Hydrodynamics and cell volume oscillations in the pollen tube apical region are integral components of the biomechanics of Nicotiana tabacum pollen tube growth. Cell Biochemistry and Biophysics 46:209-232. doi: 10.1385/CBB:46:3:209

Zonia, L., and Munnik, T. 2008. Vesicle trafficking dynamics and visualization of zones of exocytosis and endocytosis in tobacco pollen tubes. Journal of Experimental Botany 59:861-873. doi: 10.1093/jxb/ern007

Zurek, B., Sanger, J. M., Sanger, J. W., and Jockusch, B. M. 1990. Differential effects of myosin-antibody complexes on contractile rings and circumferential belts in epitheloid cells. Journal of Cell Science 97:297-306.

Zwaan, J., and Hendrix, R. W. 1973. Changes in cell and organ shape during early development of the ocular lens. American Zoologist 13:1039-1049. doi: 10.1093/icb/13.4.1039 